\title{
Prevalence and predictors of acute and chronic pain after surgery : the impatient part of the story
}

Citation for published version (APA):

Sommer, M. (2008). Prevalence and predictors of acute and chronic pain after surgery : the impatient part of the story. [Doctoral Thesis, Maastricht University]. Datawyse / Universitaire Pers Maastricht. https://doi.org/10.26481/dis.20081212ms

Document status and date:

Published: 01/01/2008

DOI:

10.26481/dis.20081212ms

Document Version:

Publisher's PDF, also known as Version of record

\section{Please check the document version of this publication:}

- A submitted manuscript is the version of the article upon submission and before peer-review. There can be important differences between the submitted version and the official published version of record.

People interested in the research are advised to contact the author for the final version of the publication, or visit the DOI to the publisher's website.

- The final author version and the galley proof are versions of the publication after peer review.

- The final published version features the final layout of the paper including the volume, issue and page numbers.

Link to publication

\footnotetext{
General rights rights.

- You may freely distribute the URL identifying the publication in the public portal. please follow below link for the End User Agreement:

www.umlib.nl/taverne-license

Take down policy

If you believe that this document breaches copyright please contact us at:

repository@maastrichtuniversity.nl

providing details and we will investigate your claim.
}

Copyright and moral rights for the publications made accessible in the public portal are retained by the authors and/or other copyright owners and it is a condition of accessing publications that users recognise and abide by the legal requirements associated with these

- Users may download and print one copy of any publication from the public portal for the purpose of private study or research.

- You may not further distribute the material or use it for any profit-making activity or commercial gain

If the publication is distributed under the terms of Article $25 \mathrm{fa}$ of the Dutch Copyright Act, indicated by the "Taverne" license above, 


\section{Prevalence and predictors of acute and chronic pain after surgery}

The inpatient part of the story

MICHAEL SOMMER

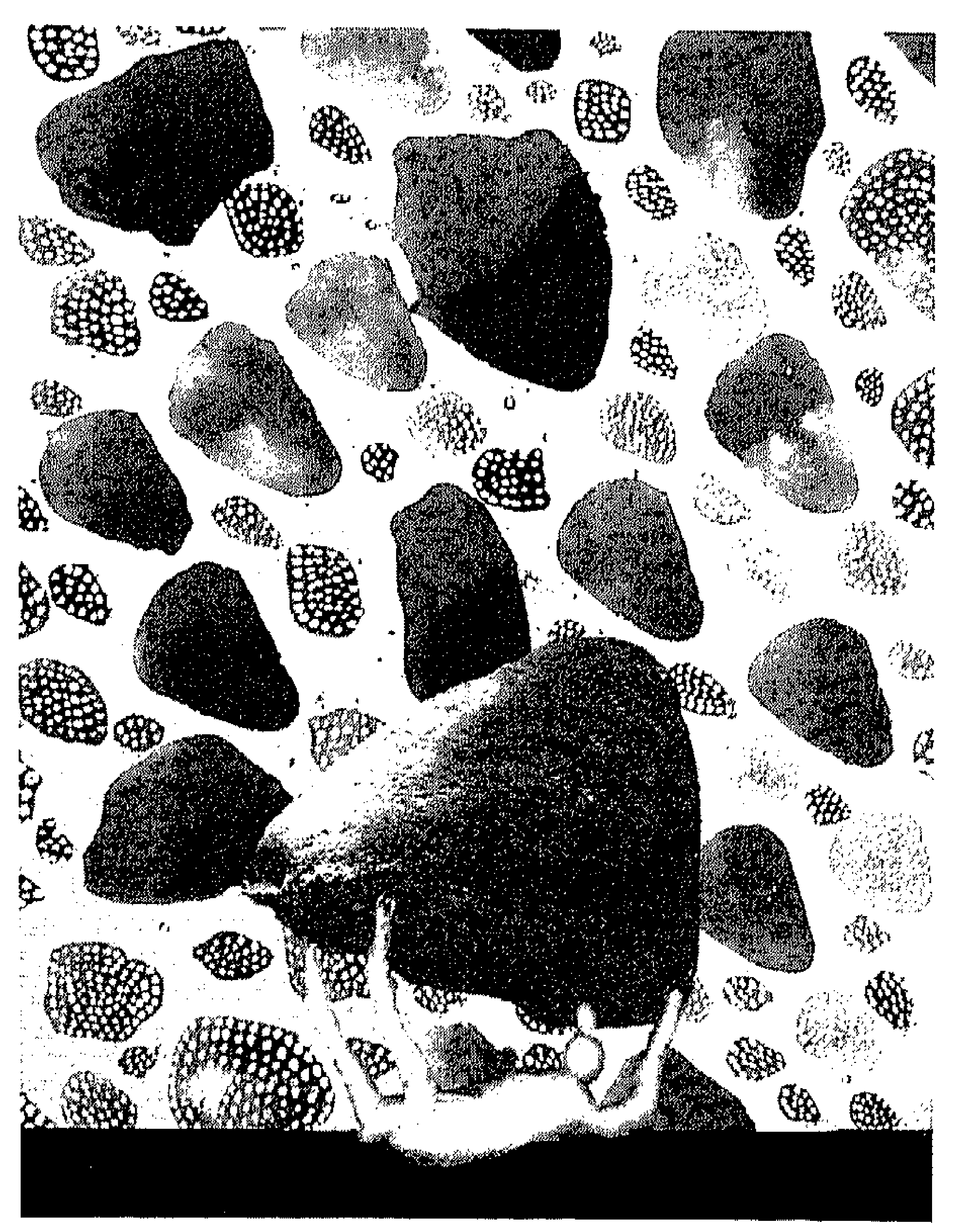


ISBN 9789052787923

C Copyright November 2008, Michael Sommer

Cover illustration: Sisyphos van Georg Kohlen, Keulen, Duitsland

Cover Design: Datawyse

Layout and print: Datawyse / Universitaire Pers Maastricht 


\title{
Prevalence and predictors of acute and chronic pain after surgery
}

\author{
The inpatient part of the story
}

\author{
PROEFSCHRIFT \\ ter verkrijging van de graad van doctor \\ aan de Universiteit Maastricht, \\ op gezag van de Rector Magnificus, prof. mr. G.P.M.F. Mols, \\ volgens het besluit van het College van Decanen, \\ in het openbaar te verdedigen \\ op vrijdag 12 december 2008 om 15.30 uur \\ door \\ Michael Sommer \\ Geboren te Darmstadt, Duitsland op 19 april 1960
}

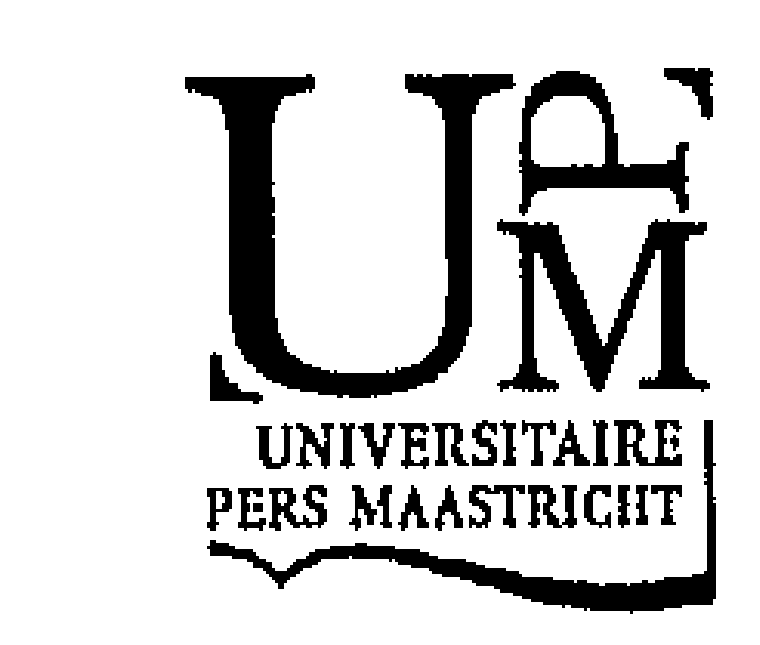




\section{Promotores}

Prof. dr. M. van Kleef

Prof. dr. A.E. Marcus (University of Münster, Germany)

\section{Beoordelingscommissie}

Prof. dr. J.H. Zwaveling (voorzitter)

Prof. dr. H. Van Aken (University of Münster, Germany)

Prof. dr. C. Neef

Prof. dr. N. Rawal (Örebro University, Sweden)

Prof. dr. L.J. Zimmermann

Prof. dr. A. van Zundert (Ghent University, Belgium) 
für Christoph 


\section{Content}

$\begin{array}{lll}\text { Chapter } 1 \text { Introduction } & 9\end{array}$

Chapter 2 The prevalence of postoperative pain in a sample of 1490 surgical inpatients

Chapter $3 \quad$ Predictors of acute postoperative pain after elective surgery 27

Chapter 4 Prevalence and predictors of postoperative pain after ear, nose 43 and throat (ENT) surgery

Chapter 5 Somatic and psychological predictors of long-term unfavourable 59 outcome after surgical intervention

Chapter 6 Predictors of unfavourable outcome after surgical intervention: 75 a 12-month follow-up study

Chapter 7 Acute pain treatment: do we need a shift in direction? 89

Chapter 8 General discussion and future perspectives 99

$\begin{array}{lll}\text { Chapter } 9 & \text { Summary } & 105\end{array}$

$\begin{array}{ll}\text { Samenvatting } & 109\end{array}$

$\begin{array}{ll}\text { Curriculum Vitae } & 113\end{array}$

$\begin{array}{ll}\text { Dankwoord } & 115\end{array}$

$\begin{array}{ll}\text { List of publications } & 117\end{array}$ 


\section{Chapter 1}

Introduction

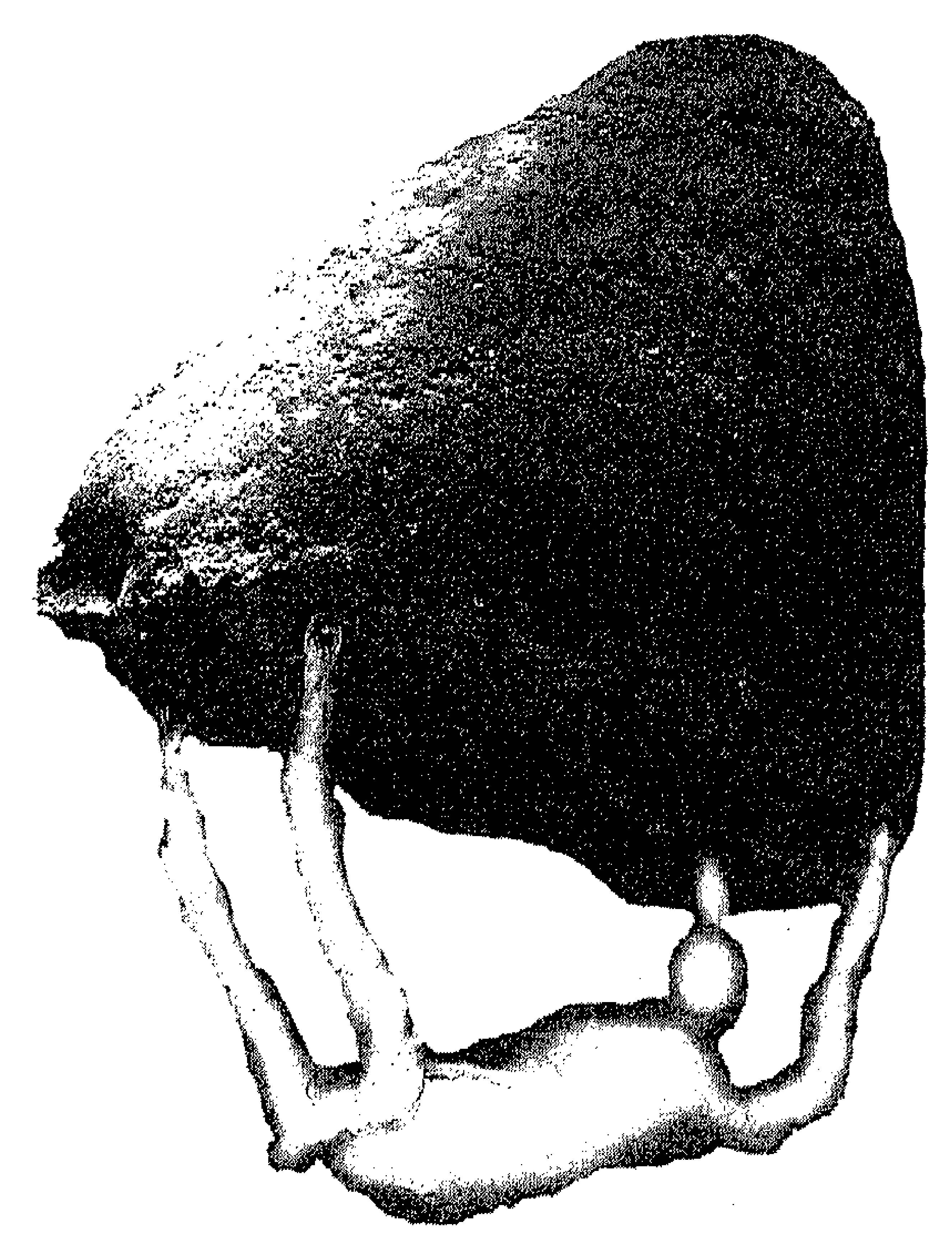




\section{CHAPTER I}

Postoperative pain is uncomfortable but more importantly, postoperative pain can probably influence short and long term medical outcome. More pain postoperatively is associated with more pulmonary and metabolic complications (1). More pain postoperatively is also a predictor of chronic pain (2). Liu and co-workers demonstrated that epidural anaesthesia during cardiac surgery could decrease pulmonary and cardiac rhythmic complications and reduce pain scores (3). This is not surprising: pain stimulates the autonomic system, which in turn leads to increased vascular resistance, tachycardia, and increased oxygen myocardial consumption. Furthermore pain reduces the diaphragmatic contractility which impairs ventilation (4). Treating pain sufficiently prevents the patient from somatic consequences of pain. A simple concept, yet we have still failed to treat pain properly.

The first studies into the number of patients with postoperative pain date from the early 1980's. Cohen et al. found a $75 \%$ incidence of severe pain (5). In 1990 this was still $75 \% 24$ hours after the operation and $65 \%$ after another 3 days (6). A lot of procedures have since been implemented in order to improve postoperative pain therapy. An Acute Pain Service (APS) was established and pain was registered at regular intervals. Recommendations, based on recent literature, were made for some surgical procedures. Continuous regional anaesthesia techniques were recommended to be applied whenever possible. Indeed things changed and postoperative incidence of moderate or severe pain declined dramatically. The incidence of postoperative pain has since been measured again. These days about $30 \%$ of all operated patients experience moderate or severe pain in the first 24 hours (7) and this further declines to $14 \%$ after 3 days. We could rest on our laurels, accept these results, and think about other, new and possibly more exciting matters requiring change. However pain is not just a matter of self-limiting discomfort. Aside from short-term effects of pain like morbidity and mortality and thus length of hospital stay, long-term effects are probably important as well. It has been recognized that insufficient pain treatment may lead to chronic pain or post surgical persisting pain (2). In the end this could have an enormous economic impact. In The Netherlands about 1.5 million procedures were carried out in the year 2006 (8). If just $1 \%$ of all operated patients got chronic pain or post surgical persisting pain we would register an increase of 15,000 patients with chronic pain per year due to surgical interventions. For some operations the estimated number of patients with chronic pain after surgery is as high as $10 \%$ (9).

To gain a better perspective on acute postoperative and chronic pain and to initiate interventions, we needed better epidemiologic and psychological data. We were interested in the incidence of postoperative pain in a broad hospital population, which was treated following modern pain guidelines and using modern drugs and techniques. Of the patients experiencing moderate or severe pain we analysed the relationship between pain and anatomical region or operative procedure. Rawal categorized operations into three groups: minor, intermediate, and major with respect to expected postoperative pain (10). Is the amount of anticipated postoperative pain per operation correct if the Rawal approach is applied as a guideline? 
The second step was to look for predictors of patients at risk. What makes a person susceptible to more pain after an intervention? Are there any factors that expose a patient to a higher risk of suffering pain? These could be procedural factors like anaesthesia techniques, anatomical region, time duration of operation, or patient-based factors such as pain before surgery, expected pain postoperatively, anxiety, pain catastrophizing or others.

Thirdly, we studied a uniform patient population from one surgical department (ear nose throat surgery). We evaluated our data in this subpopulation once again with respect to prevalence of postoperative pain and predictors of patients at risk. We were curious if we would see results similar to that of a huge database in a smaller subpopulation?

The relationship between acute and chronic pain is intriguing and seems to be very important. Currently there are only speculations about the pathophysiologic background. Central sensitization could play a role in this process due to ongoing neuropathic changes or toxic stimulations. The incidence in relation to psychological profiles is not known (11). It was necessary to first evaluate incidence of chronic pain after surgery before identifying the risk factors of chronic pain. Our patients were interviewed a second time, after 6 months, and a third time, after 12 months, to register the grade of pain after surgery on a longer term and to evaluate whether there is pain after surgery at all and, secondly, whether we could detect risk factors of chronic pain.

In summary in this thesis the following research questions were studied:

- What is the incidence of acute moderate or severe pain after surgery in a huge and mixed patient population from different surgical departments in a hospital with an APS?

- Can somatic and/or psychological predictors be identified of patients at risk with respect to moderate or severe postoperative pain?

- What is the prevalence and what are the predictors of acute moderate or severe pain in a subpopulation?

- What is the incidence of chronic pain in patients 6 months after surgery and are there any predictors of chronic pain?

- What is the incidence of chronic pain in patients 12 months after surgery and are there any predictors of chronic pain? 


\section{REFERENCES}

1. Liu SS, Carpenter RL, Mackey DC et al. Effects of perioperative analgesic technique on rate of recovery after colon surgery. Anesthesiology 1995;83:757-65.

2. Macrae WA. Chronic pain after surgery. Br J Anaesth 2001;87:88-98.

3. Liu SS, Block BM, Wu CL. Effects of perioperative central neuraxial analgesia on outcome after coronary artery bypass surgery: a meta-analysis. Anesthesiology 2004;101:153-61.

4. Desai PM. Pain management and pulmonary dysfunction. Crit Care Clin 1999; 15:151-66, vii.

5. Cohen FL. Postsurgical pain relief: patients' status and nurses' medication choices. Pain 1980;9:265-74.

6. Owen H, McMillan V, Rogowski D. Postoperative pain therapy: a survey of patients expectations and their experiences. Pain 1990;41:303-7.

7. Dolin SJ, Cashman JN, Bland JM. Effectiveness of acute postoperative pain management: I. Evidence from published data. $\mathrm{Br} J$ Anaesth 2002;89:409-23.

8. www.prismant.nl//nformatie-expertise/Thema's/Ziekenhuisstatistieken, 2008

9. Kehlet $H$, Jensen TS, Woolf CJ. Persistent postsurgical pain: risk factors and prevention. Lancet 2006;367:1618-25.

10. Rawal N. Postoperative pain and its management London: BMJ Books, 1989.1

11. Joshi GP, Ogunnaike BO. Consequences of inadequate postoperative pain relief and chronic persistent postoperative pain. Anesthesiol Clin North America 2005;23:21-36. 


\section{Chapter 2}

\section{The prevalence of postoperative pain in a sample of 1490 surgical inpatients}

Michael Sommer MD, Janneke M. de Rijke PhD, Maarten van Kleef MD PhD,

Alfons G.H. Kessels MD M.Sc., Madelon L. Peters PhD, José W.J.M. Geurts M.Sc.,

Hans-Fritz Gramke MD, Marco A.E. Marcus MD PhD

Published European Journal of Anesthesiology 2008; 25: 267-274

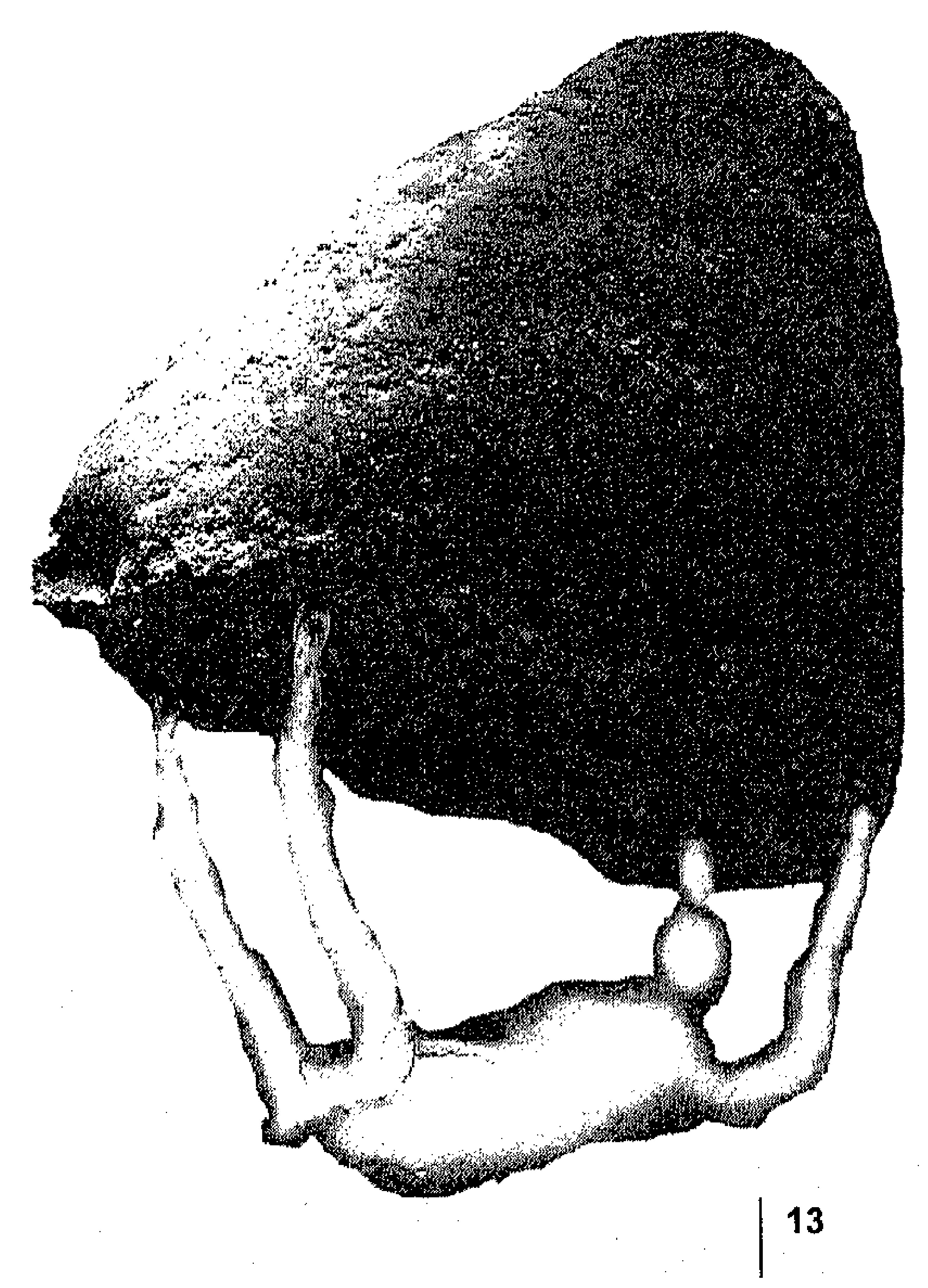




\section{ABSTRACT}

To measure the prevalence of postoperative pain, an assessment was made of 1490 surgical inpatients that were receiving postoperative pain treatment according to an acute pain protocol. Measurements of pain (scores from 0-100 on a Visual Analogue Scale (VAS)) were obtained (three times a day) on the day before surgery and on days 0-4 postoperatively; mean pain intensity scores were calculated. Patients were classified as having no pain (VAS $0-5 \mathrm{~mm})$, mild pain $(6-40 \mathrm{~mm})$, moderate pain (41$74 \mathrm{~mm})$ or severe pain $(75-100 \mathrm{~mm})$.

Moderate or severe pain was reported by $41 \%$ of the patients on day $0,30 \%$ on day 1 and 19,16 and $14 \%$ on day 2,3 , and 4 , respectively. The prevalence of moderate or severe pain in the abdominal surgery group was high on post-operative days $0-1$ (POD's $0-1)(30-55 \%)$. A high prevalence of moderate or severe pain was found during the whole of POD's 1 to 4 in the extremity surgery group (20-71\%) and in the back/spinal surgery group (30-64\%). We conclude that despite the acute pain protocol, postoperative pain treatment was unsatisfactory, especially after intermediate and major surgical procedures on an extremity or on the spine.

\section{Keywords:}

Pain, prevalence, postoperative, protocol, surgery, indicator 


\section{INTRODUCTION}

During the past two decades, a great deal of attention has been paid to postoperative pain. Although the first suggestions were made in 1976 to introduce an "analgesic team" within hospitals to improve postoperative pain management (1), it took until 1988 before the first official guidelines were published on the treatment of postoperative pain (2). Rawal advocated a "stepwise approach" to postoperative pain, in which operations were classified as being minor, intermediate or major depending on the anticipated level of postoperative pain (3).

The question arises as to whether these guidelines and the subsequent introduction of acute pain teams have led to improvements in patient care in the postoperative period. According to the existing literature, there has been very little improvement in postoperative pain treatment over the past two decades (4-9). However, it is difficult to compare the results between studies, due to differences in design, sample selection, sample size, pain evaluation assessment instruments, choice of pain intensity designation (mean, median or percentage) and especially due to poor descriptions of the findings. Postoperative pain is accepted as being a common clinical prob$\mathrm{lem}$, but if more detailed information is required, for example, which surgical procedures are associated with the most postoperative pain, surprisingly few data are available in the literature. To improve pain protocols, it is important to have detailed knowledge.

Quite recently, the measurement of pain in a routine, standardized and easily comparable manner has become even more important in The Netherlands, because new Dutch healthcare regulations stipulate that the quality of the services must be made public by means of quality indicators. Searches have been made for quality indicators in different specialties and in many countries (11). The purpose of these quality indicators, which are being developed by governments and researchers, is to measure and monitor the performance of health care providers. Patients and insurance companies can then choose hospitals on the basis of these indicators. In 2004, a quality indicator for postoperative pain was formulated in The Netherlands as being the number of patients with a pain score of lower than 4 (on a scale 0-10) in the first 72 hours after surgery divided by the number of patients evaluated. At present, however, only a small number of hospitals can publish complete data, because standardized pain registration has not yet been implemented at the majority of Dutch hospitals (13).

In preparation for the implementation of a standardized pain-registration programme, we wanted to know the proportion of patients who have unacceptable postoperative pain scores at our hospital, with 700 beds, 20,000 surgical procedures per year and an acute pain management protocol that has been in use since 1995. Therefore, independent researchers measured the levels of postoperative pain in 1490 surgical inpatients and made an inventory of the most painful operation sites. 


\section{PATIENTS AND METHODS}

A prevalence study was carried out on days 0-4 postoperatively to obtain short-term follow-up data on surgery-related pain. After receiving approval from the institutional ethics committee, we enrolled all the consecutive patients admitted to the University Hospital Maastricht, the Netherlands, who were scheduled to undergo elective surgery between 1 February to 30 August 2003. The following surgical departments took part: general-, plastic-, ear nose and throat-, facio-maxillary-, neuro- and thoracic surgery, orthopaedics, ophthalmology, gynaecology and urology. Patients were excluded from the study if they were younger than 18 years, had limitations of selfexpression, visual dysfunction, insufficient knowledge of the Dutch language, or had been admitted for acute surgery, cardiac surgery or caesarean section, or had required postoperative ventilatory support. A total of 1975 subjects were eligible, of which $1663(85 \%)$ agreed to participate. There were $140(8 \%)$ drop-outs for various reasons: postoperative ventilatory support $(n=50)$, the study became too burdensome for the patient $(n=32)$, complications followed by a second operation $(n=14)$ and miscellaneous $(n=44)$. The questionnaires from $33(2 \%)$ patients were not evaluable, because of too many missing data, which left 1490 evaluable patients. There were no significant differences in age and gender distributions between the non-responders and the participants.

After admission to the surgical ward, a trained research assistant explained the purpose and methods of the study to each eligible patient. Socio-demographic variables were recorded on all the patients who were willing to participate in the study. Pain intensities at rest and while coughing were scored one and three hours postoperatively and at 9 P.M using a $100 \mathrm{~mm}$ visual analogue scale (VAS) anchored to "no pain" and "worst pain I can imagine". On days 1-4 after the operation, pain was scored in a pain diary, three times a day. A research assistant visited all the patients once a day to give help if necessary, or three times a day if they were unable to fill in the diary themselves. The type of anaesthesia was not regulated by the study protocol. The choice of administration technique and analgesic agent was left to the individual anaesthetist and patient. (Agents used for general anaesthesia were propofol, etomidate, sufentanil, fentanyl, rocuronium, and vecuronium). Patients who were discharged from hospital within four days postoperatively took their diary home and returned it to us in a special pre-paid envelope. Diaries that had not been returned within 14 days after surgery were chased up by contacting the patient by phone.

The perioperative pain protocol that has been used at our hospital since 1995 is based on the stepwise approach of acute pain treatment described by Rawal (3). All the operations were categorized into three groups (minor, intermediate and major surgery) based on the anticipated level of postoperative pain. Subsequently, all the surgical procedures were categorized according to the anatomical site. This classification is also used in chronic pain patients and it has been recommended in the recent literature (14) (Table 1). Our preoperative protocol ensured that all the patients received paracetamol orally or rectally one hour before the induction of anaesthesia. 
In agreement with the prevailing protocol, postoperative pain after minor operations was treated with paracetamol (1000 mg) four times a day, combined with nonsteroidal anti-inflammatory drugs (NSAIDs) administered by the ward nurses. Rescue medication for moderate or severe pain (VAS>40) was piritramide intramuscularly (i.m.). Pain after intermediate operations was treated using the same protocol, combined with intravenous (i.v.) piritramide $(2-5 \mathrm{mg})$, which was repeated until the patient reported being pain-free. This was followed by i.m. piritramide $10-15 \mathrm{mg}$ six times a day. After major operations, piritramide was administered by intermittent intravenous infusion on an "as needed" basis by the patient (Patient Controlled Analgesia, PCA), in line with the PCA protocol that has three dose levels of piritramide $(1.0,1.5$ and 2 $\mathrm{mg}$ per bolus, with a lock-out interval of 5 minutes). In many cases, major operations were conducted with a combination of general anaesthesia and epidural anaesthesia. Postoperatively, pain was treated by the continuous epidural infusion of bupivacaine $0.125 \%$ with sufentanil $1 \mu \mathrm{g} / \mathrm{ml}$.

After a post-anesthesia care unit observation period of 2-4 hours, patients who had received minor and intermediate surgery returned to the surgical ward under the care of nurses who have experience with the postoperative pain protocol. Patients who underwent major surgery were followed by a specially trained postoperative pain nurse at least twice a day during the first 4 postoperative days. They enquired about pain at rest and after movement. If the pain score was higher than 40 and the patient had an epidural catheter for the continuous administration of analgesics, they administered a bolus of $5 \mathrm{cc}$ and waited another 15 minutes. After sufficient pain relief they increased the continuous analgesic level by $2 \mathrm{cc} /$ hour and revisited the patient 2 hours later. If there was no effect of bolus application, they contacted the anaesthetist who was supervising the pain team. When the patient was on PCA treatment, the first step was to find the reason for failure (bolus too low, lockout time too long, other reason) and treat it. Then the effect was evaluated and after the pain score had decreased, they returned two hours later to re-evaluate the pain treatment. If there was no improvement in the pain scores, they administered an extra bolus and increased the PCA bolus by one step according to the protocol and re-evaluated the patient 2 hours later. Pain treatment was delivered in strict conformity with our protocol to every patient whether they were included in the study or not.

Data were analysed using descriptive statistics. Actual pain scores (VAS 0-100 $\mathrm{mm}$ ) on the day of the operation (POD 0) were used, measured at 1 hour and 3 hours postoperatively and at $9.00 \mathrm{p} . \mathrm{m}$. Mean pain scores on the day of the operation and on postoperative days 0 to 4 (POD 1-4) were calculated, using the average of the three scores obtained from each individual on each of the days. As the pain diaries were sometimes incomplete, totals could vary from day to day. A mean pain score of higher than $40 \mathrm{~mm}$ on a VAS was regarded as being unacceptable. We defined $0-5$ (VAS 0-100 mm) as no pain, 6-40 $\mathrm{mm}$ as mild pain, $41-74 \mathrm{~mm}$ as moderate pain and $75-100 \mathrm{~mm}$ as severe pain $(8,15,16)$. 


\section{RESULTS}

Analyses were performed on the data from a total of 1490 evaluable patients. Table 1 shows the distribution of the operations subdivided into the anticipated level of pain and anatomical site. Patient characteristics are presented in table 2. Slightly fewer male patients participated in this study than female patients (47\% versus $53 \%$ ). Forty-five per cent of the patients were 60 years of age or older.

In figure 1, VAS-scores show that 1 hour after the operation 357 out of 1361 evaluable patients (26\%) were suffering from moderate pain at rest (VAS 41-74; white section, first column) and $210(15 \%)$ were suffering from severe pain at rest (VAS>75; black top section, first column). Three hours after the operation and at 9.00 p.m., these percentages were $26 \%$ and $10 \%$ for moderate and severe pain, respectively.

The figure shows also that 1 day after surgery 395 (30\%) out of the remaining 1306 patients reported moderate (white section, fourth column) or severe (black top section, fourth column) pain at rest (VAS $>40 \mathrm{~mm}$ ). On postoperative days 2, 3 and 4 , these percentages were $19 \%, 16 \%$ and $14 \%$, respectively .

The proportion of patients with moderate or severe pain increased while coughing in all anatomical subdivisions, except for the lower extremity (POD 1)(figure 2). Pain levels increased by $1 \%$ in the head/neck surgery patients to $21 \%$ in the upper abdominal surgery patients. In the lower extremity subdivision, pain while coughing decreased by $6 \%$.

Table 2 also shows the proportions of patients with a pain level of $>40$ at rest (VAS) in relation with their demographic and clinical characteristics. Significantly higher proportions of patients with moderate or severe pain were younger than 60 years, were female and had received general anaesthesia only (day of operation). Surprisingly, from POD 1, the highest pain scores were found in the patients who had received general anaesthesia in combination with a loco-regional technique. Shortly after the operation, the patients in the intermediate surgery group had significantly more pain than the patients who had undergone minor or major surgery. On POD's 1 to 4 , the patients who had received major surgery had the highest pain scores and this also applied to the patients whose operations had taken more than 2 hours.

Table 3 shows the distribution of patients with moderate or severe pain at rest (VAS $>40 \mathrm{~mm}$ ) per anatomical site and the anticipated pain level of the operation (minor, intermediate and major). The prevalence of moderate or severe pain in the abdominal surgery groups was high on the day of surgery and on POD 1 (30-55\%), except for the lower abdomen/pelvis surgery group (minor) $(20 \%$ and $11 \%$ ). High prevalences of moderate or severe pain at rest were found during the whole of POD's 1 to 4 in the upper/lower extremity group (20-71\%) as well as in the back/spinal group $(30-64 \%)$. 
Wratal

Table 1. Surgical procedures subdivided according to anticipated postoperative pain level and anatomical site.

\begin{tabular}{|c|c|c|}
\hline $\begin{array}{l}\text { Anticipated } \\
\text { pain level }\end{array}$ & Anatomical site & Surgical procedure \\
\hline \multirow[t]{6}{*}{ Minor } & $\begin{array}{l}\text { Head and neck } \\
n=295\end{array}$ & $\begin{array}{l}\text { Thyroidectomy, stapedectomy, tympanoplasty, petrosal bone } \\
\text { and middle ear surgery, cochlear implantation, middle ear } \\
\text { inspection, middle ear reconstruction, auricle reconstruction, } \\
\text { nasal sinus inspection, endoscopy of pharynx, larynx, bronchus, } \\
\text { intracranial tumour surgery, craniotomy, eye surgery }\end{array}$ \\
\hline & $\begin{array}{l}\text { Upper extremity } \\
n=10\end{array}$ & Peripheral vascular operations, wound toilet, sutures, \\
\hline & $\begin{array}{l}\text { Thorax- noncardiac } \\
n=10\end{array}$ & Vascular operations, wound toilet, \\
\hline & $\begin{array}{l}\text { Lower abdomen/pelvis } \\
n=138\end{array}$ & $\begin{array}{l}\text { Vaginal urological procedures, cervix operation, abortion, hys- } \\
\text { teroscopy, operations of male genital, endoscopic urological } \\
\text { interventions e.g. TUR, cystic biopsy, urethra, cystoscopy, } \\
\text { plastic skin operations }\end{array}$ \\
\hline & $\begin{array}{l}\text { Lower extremity } \\
\mathrm{n}=20\end{array}$ & $\begin{array}{l}\text { plastic skin operations, peripheral vascular operations, wound } \\
\text { toilet }\end{array}$ \\
\hline & $\begin{array}{l}\text { Back/Spinal } \\
n=13\end{array}$ & Urological neuromodulation, small plastic skin operations, \\
\hline \multirow[t]{8}{*}{ Intermediate } & $\begin{array}{l}\text { Head and neck } \\
n=85\end{array}$ & $\begin{array}{l}\text { Neck dissection, mouth and throat surgery, laryngectomy, } \\
\text { pharynx and larynx surgery, maxilla and mandibular surgery }\end{array}$ \\
\hline & $\begin{array}{l}\text { Upper extremity } \\
n=39\end{array}$ & Orthopaedic hand and arm surgery, \\
\hline & $\begin{array}{l}\text { Thorax- noncardiac } \\
\mathrm{n}=31\end{array}$ & Oncological and plastic breast surgery \\
\hline & $\begin{array}{l}\text { Upper abdomen } \\
n=69\end{array}$ & $\begin{array}{l}\text { Fundoplication, duodenum surgery, cholecystectomy, nephrec- } \\
\text { tomy, }\end{array}$ \\
\hline & $\begin{array}{l}\text { Lower abdomen/pelvis } \\
\mathrm{n}=195\end{array}$ & $\begin{array}{l}\text { Surgery of vulva, ovary, adnexen, vaginal and abdominal uter- } \\
\text { usextirpation, abd. endometriosis, closure of anus praeter, } \\
\text { colorectal surgery, anal surgery, abdominal vascular surgery, } \\
\text { abd. lipectomy, cystic resection, Ryntshak and radical prostatec- } \\
\text { tomy }\end{array}$ \\
\hline & $\begin{array}{l}\text { Lower extremity } \\
n=212\end{array}$ & $\begin{array}{l}\text { Plastic skin operations, orthopaedic surgery foot/knee/leg/hip, } \\
\text { amputation, peripheral vascular surgery }\end{array}$ \\
\hline & $\begin{array}{l}\text { Back/spinal } \\
n=63\end{array}$ & $\begin{array}{l}\text { Plastic skin operations, spinal cord decompression with hemi- } \\
\text { laminectomy, discectomy }\end{array}$ \\
\hline & $\begin{array}{l}>\text { than } 1 \text { site } \\
n=12\end{array}$ & Plastic breast + abdominal surgery, arm/hand and hip (fractures) \\
\hline \multirow[t]{5}{*}{ Major } & $\begin{array}{l}\text { Upper extremity } \\
\mathrm{n}=23\end{array}$ & Shoulder surgery (endoprothesis), clavicle surgery \\
\hline & $\begin{array}{l}\text { Thorax- noncardiac } \\
\mathrm{n}=10\end{array}$ & Lobectomy \\
\hline & $\begin{array}{l}\text { Upper + lower abdomen } \\
\mathrm{n}=171\end{array}$ & $\begin{array}{l}\text { Hepatectomy, pancreatectomy, combination of different bowel } \\
\text { surgery, aortic surgery, extensive gynaecological surgery, } \\
\text { combination of bowel and gynaecological surgery }\end{array}$ \\
\hline & $\begin{array}{l}\text { Lower extremity } \\
n=60\end{array}$ & Total knee replacement \\
\hline & $\begin{array}{l}\text { Back/spinal } \\
\mathrm{n}=34\end{array}$ & Spondylodesis, spinal cord tumour resection, untethering \\
\hline
\end{tabular}




\section{CHAs?}

Table 2. Distribution of patient characteristics in the total sample (column 2) and the number ( $n$ ) and proportion (\%) of patients with pain at rest $>40$ (VAS) on the day of the operation ( 1 and $3 \mathrm{~h}$ postoperatively (PO) and at 9.00 p.m.) and with a mean pain at rest of $>40$ (VAS) 4 days postoperatively (PODs $1-4$ ) in relation with patient characteristics.

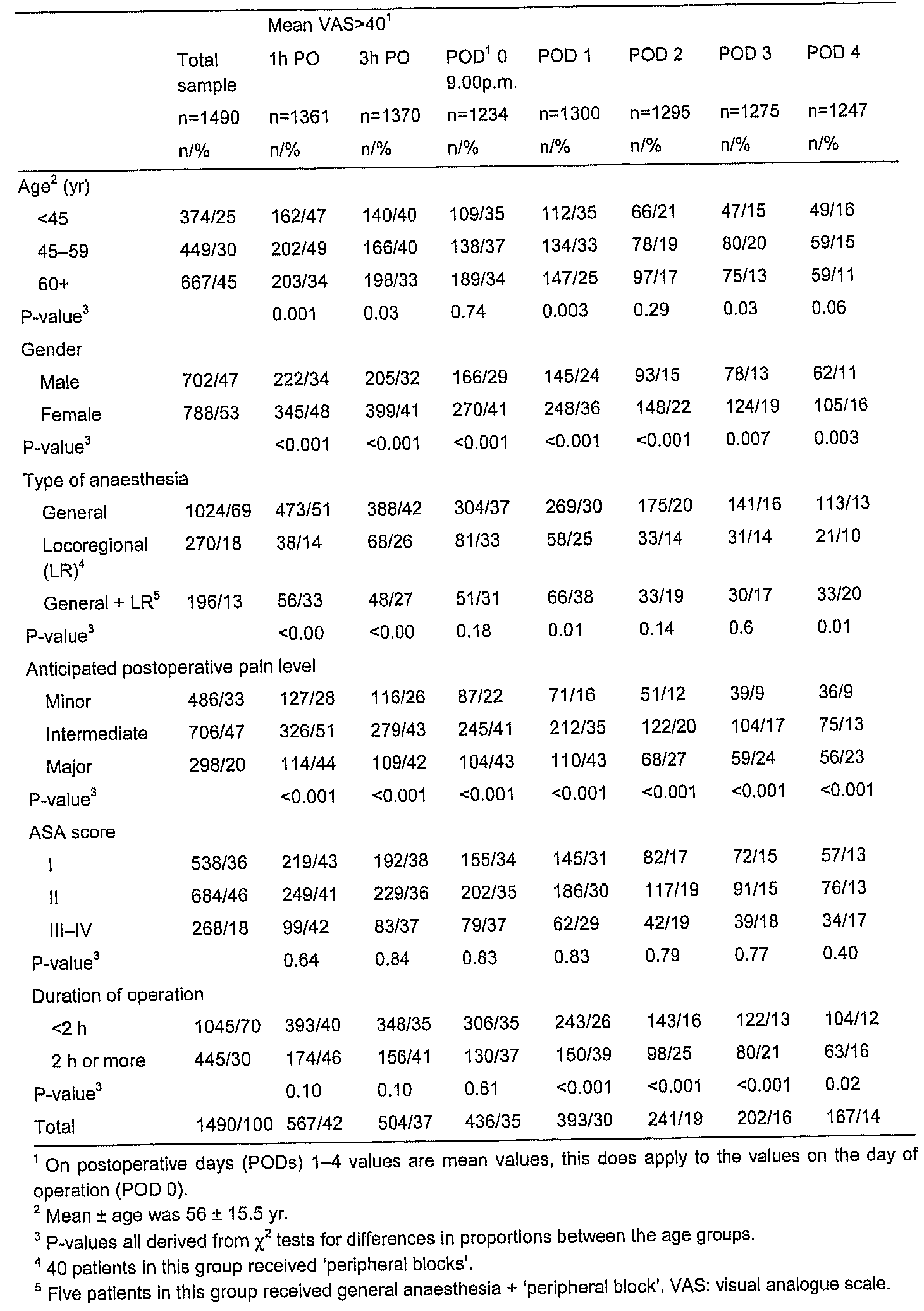


PREVIAENCE OF POSTOPERATUE PAN

Table 3. Number $(n)$ and proportion (\%) of patients with pain at rest $>40$ (VAS) on the day of surgery ( 1 and 3 $h$ postoperatively and at $9.00 \mathrm{p} . \mathrm{m}$.) and with a mean pain at rest of $>40$ (VAS) 4 days postoperatively (PODs $1-4)$ by anatomical site and anticipated postoperative pain level (minor, intermediate, major).

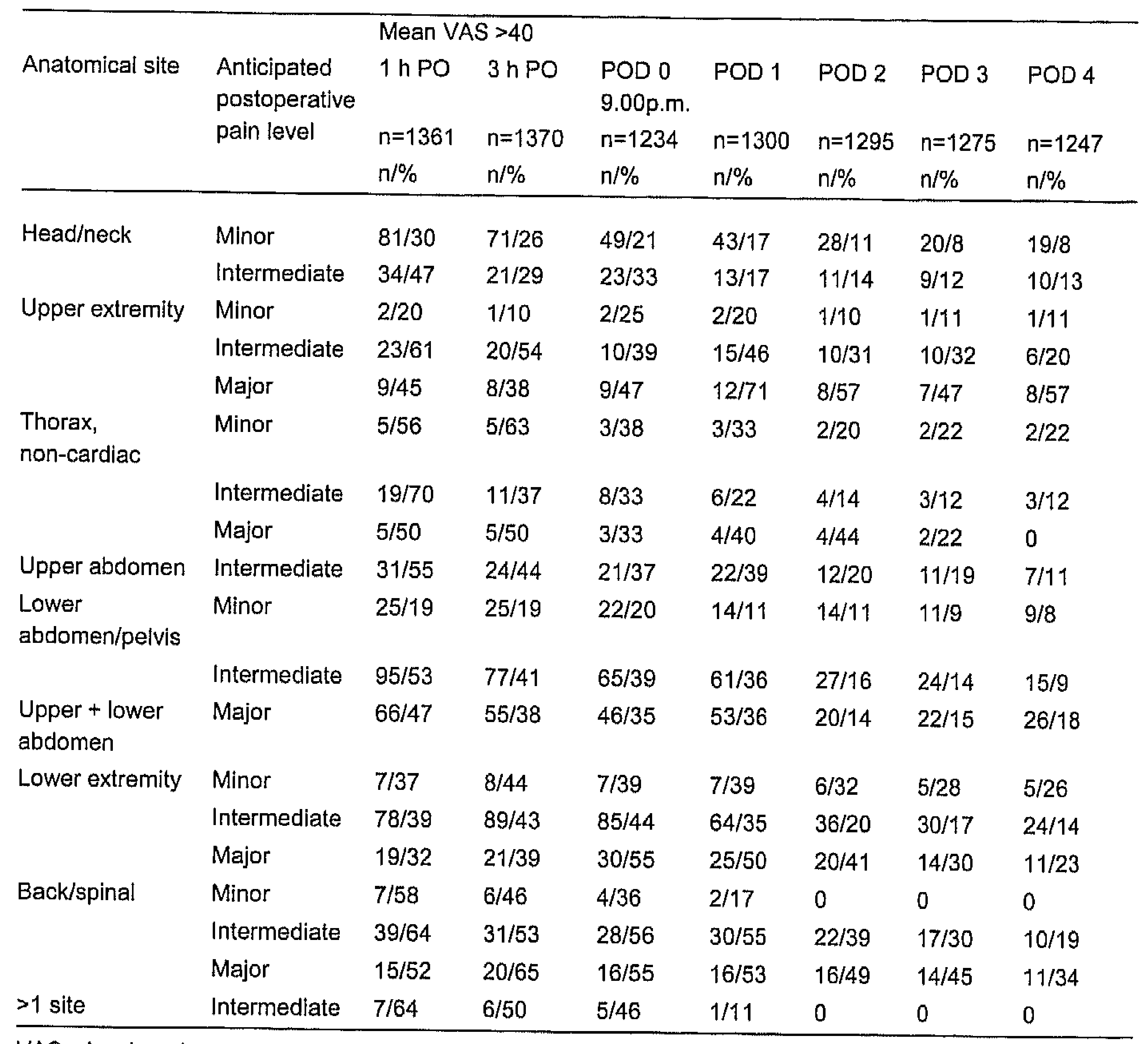

VAS: visual analogue scale; PO: postoperatively; PODs: postoperative days. 
CHAPY?

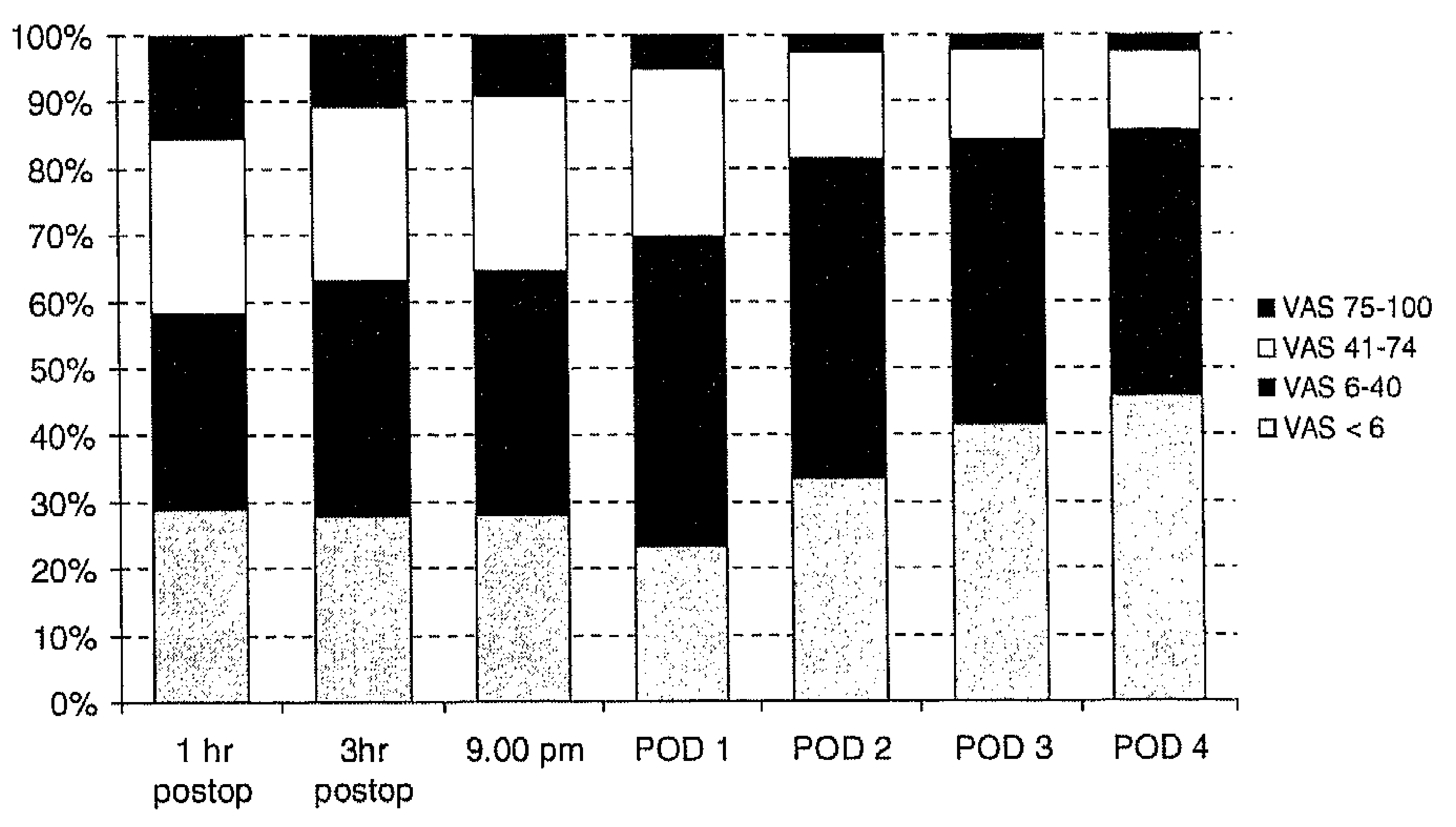

Figure 1. Distribution of pain at rest (visual analogue scale) on the day of surgery ( 1 and $3 \mathrm{~h}$ postoperatively and at 9.00p.m.) and mean pain on postoperative days (PODs) $1-4$.

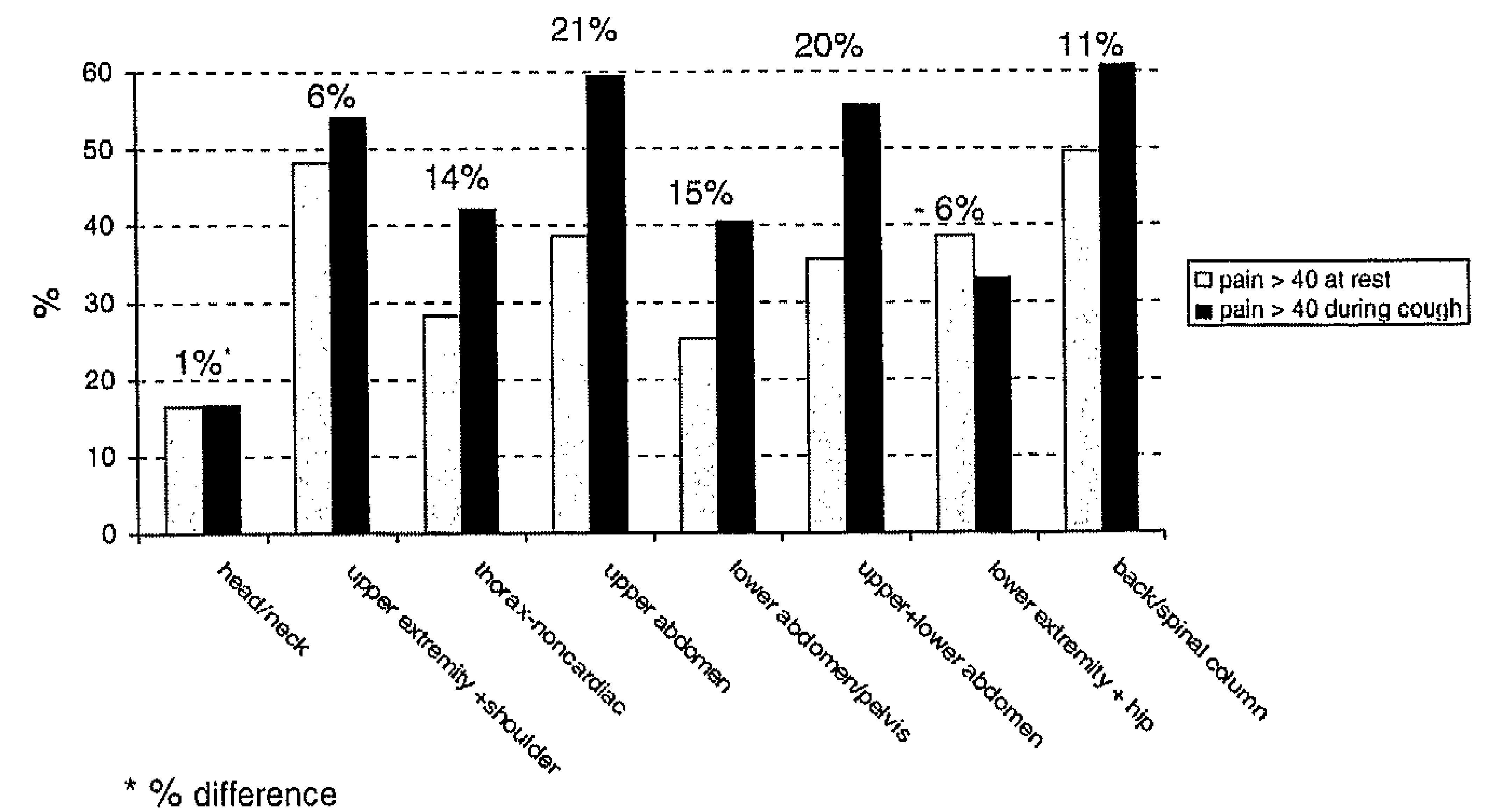

Flgure 2. Distribution of patients with a mean pain at rest of 40 (visual analogue scale, VAS) and mean pain during coughing of .40 (VAS) on postoperative day 1 in relation with anatomical site. 


\section{DISCUSSION}

We assessed the prevalence of postoperative pain in 1490 surgical inpatients who were receiving postoperative pain treatment according to an established acute pain protocol. Moderate or severe pain at rest was reported by $40 \%$ of the patients on the operation day. It than declined from day 2 until day 4 , but almost $15 \%$ of all patients still had moderate or severe pain on day 4. Prevalence of moderate or severe pain at rest was high on the day of surgery and on POD $1(30-55 \%)$ in the abdominal surgery group. The highest prevalences of moderate or severe pain at rest during the whole of POD's 1 to 4 were found in the patients who had undergone operations on the upper (intermediate and major) or lower (minor and major) extremity, or operations on the back/spine (intermediate and major), which had mainly comprised hemilaminectomy and spondylodesis.

In 1980, Cohen and colleagues (7) found an incidence of $75 \%$ moderate or severe unrelieved pain. A survey by Owen and colleagues $(n=259)$ in 1990 showed that 24 hours after the operation, $75 \%$ were experiencing moderate to unbearable pain (Verbal Rating Scale), while 72 hours afterwards this was $65 \%$ (17). No information was given about the anticipated pain level or anatomical site of the surgical procedures. Apparently, very few studies described the prevalence of postoperative pain in terms of anatomical site and anticipated pain level of the operation. In 1994, Oates and colleagues reported a prevalence of $34 \%$ with moderate or severe pain (VAS $>=6$ ) in a group of 199 patients who had undergone various surgical procedures (5). Svensson and colleagues (2000) measured pain levels in 185 patients (surgical groups hip, knee, back, urological and gastrointestinal) (6). At 4, 24, 48 and 72 hours after the operation, moderate or severe pain (VAS > 40) at rest was experienced by $39 \%, 43 \%, 27 \%$ and $16 \%$ of the patients, respectively. These proportions were slightly higher than our findings. Unfortunately, no information was given about the postoperative pain level in relation with the surgical procedure.

In a review (2002) of data published on the incidence of moderate to severe or severe pain after major surgery, mean $(95 \% \mathrm{Cl})$ incidences of moderate to severe pain and severe pain within the first 24 hours were $30 \%(26-33 \%)$ and $11 \%(8-13 \%)$, respectively (8). In the review, moderate to severe pain was defined as a VAS score of higher than $30 / 100$, or a numerical score of more than $3 / 10$. Severe pain was defined as a pain intensity score in excess of $70 / 100$ or $7 / 10$. In our study, $43 \%$ of the patients who underwent major surgery experienced moderate or severe pain on day 1. This rate falls within the $95 \%$ confidence interval found by Dolin and colleagues, although we used a VAS cut-off point of $>40 \mathrm{~mm}$.

We also measured pain while coughing as an indicator of pain during movement. Although coughing was not the most ideal option, it was the most feasible manoeuvre in a large and heterogeneous group of surgical patients. Pain increased in all the surgical groups, except for the lower extremity. An increase in pain during movement is unacceptable, because it prevents the patient from being mobilized, or from breathing adequately. Some authors therefore recommended prophylactic treatment when pain levels increased beyond 40 on the VAS at former exercise sessions (18). A 


\section{CHAPTER?}

decrease in pain intensity during coughing has been described before in a patient group that underwent hip operations (19). The authors suggested that the decrease in pain could be due to distraction, which is a cognitive method of pain reduction. Another explanation for this phenomenon might be segmental inhibitory pathway activation (20).

In our study, the most painful surgical procedures (moderate pain: VAS 40 -74 and severe pain: VAS>75) were upper and lower extremity, thorax, abdomen and back/spinal column surgery. Upper extremity operations and thorax surgery with incision of the pleura were classified in the major surgery category, so advanced pain treatment had to be considered. However, a proportion of the breast surgery and back surgery patients had been included in the intermediate surgery group, in which they had mainly received intramuscular bolus injections of opioids. This corresponds with one of the conclusions drawn in the review by Dolin and colleagues, namely that the highest percentage of patients with inadequate pain relief were receiving i.m. analgesics (8). The authors argued that when i.m. analgesia is administered using strict criteria, it can be an effective technique. However, the literature strongly suggests that this is not the case in clinical practice. It might be worthwhile to assign these patients to the major surgery group, but even the major surgery patients had high pain scores. There are several possible explanations for this. Firstly, a small proportion of the high levels of pain in the major surgery group might be explained by failure of the epidural catheters, which had an incidence of $5.7 \%(8)$. Secondly, a proportion may have been due to suboptimal pain management on the hospital ward. Rawal argued that the presence of an APS at a hospital does not automatically mean that all the patients receive good analgesia and are satisfied (14). Stamer and colleagues conducted a survey on the organization and quality of acute pain services in Germany (21). They defined basic quality criteria: personnel assigned to provide APS, night and weekend policies, written pain management protocols and regular assessments and registration of pain scores at least once a day. It was found that $50 \%$ of German AP Services did not comply with these quality criteria. At our hospital, regular pain score assessments are only done on patients with a continuous epidural infusion or PCA. It is likely that the extension of standardized pain score documentation to all postoperative patients will contribute to more optimal pain management on hospital wards.

Thirdly, the acute pain protocol itself might have been insufficient if the estimate of anticipated postoperative pain was too low. In 2003, a multidisciplinary task force formulated postoperative pain guidelines after reviewing and evaluating the current literature on evidence-based knowledge, in conformity with the Agency for Health Care Policy and Research guideline published in 1993 (22). All aspects of pain treatment were included, e.g. systemic oral, i.m., i.v., regional and non-medicinal techniques. In their recommendations, the anatomical site of the operation is an important aspect in decision-making concerning analgesic treatment. In a table, the choice of analgesia technique is correlated with the site of surgery or intervention. An algorithm to follow specific operations that incorporates the influence of the duration of surgery and the degree of tissue damage could make a valuable contribution to 
the standard pain protocol. In our subgroup analyses, we found significantly higher proportions of patients with moderate or severe pain among the younger patients $<$ 60 years), females and patients who had received general anaesthesia only. This also applied to intermediate and major surgery and to operations that had taken more than 2 hours. These factors were exactly the same as those found by Kalkman and colleague as being predictive for severe postoperative pain shortly after awakening. In addition, these factors have been identified as risk factors for chronic postoperative pain (23-25). Besides the influence of various "demographic" and general medical aspects, it has been proposed that individual psychological factors also influence postoperative pain, such as preoperative anxiety (26) and pain catastrophizing (27).

For a couple of surgical interventions, there are recommendations with respect to postoperative pain treatment given by the PROSPECT task force (10). These recommendations, which are based on a recent literature review, are revised every two years. It is desirable that these recommendations will be part of the daily medical practise in each hospital. On the other hand, a lot more operations must be tackled by the task force in the future. Moreover, more data about somatic, psychological, demographic as well as genetic factors must be collected to get a deeper insight in the relation between surgical intervention and acute pain. 


\section{WHATER 2}

\section{REFERENCES}

1. Anonymous. Editorial: Postoperative pain. Anaesthesia and intensive care 1976;4:95.

2. Rowlingson JC, Rawal N. Postoperative pain guidelines-targeted to the site of surgery. Regional anesthesia and pain medicine 2003;28:265-7.

3. Rawal N. Postoperative pain and its management. In: Rawal N, ed. Management of acute and chronic pain London: BMJ Books, 1989

4. Donovan $M$, Dillon P, McGuire L. Incidence and characteristics of pain in a sample of medical-surgical inpatients. Pain 1987;30:69-78.

5. Oates JD, Snowdon SL, Jayson DW. Failure of pain relief after surgery. Attitudes of ward staff and patients to postoperative analgesia. Anaesthesia 1994;49:755-8.

6. Svensson I, Sjöström B, Haljamäe H. Assessment of pain experiences after elective surgery. Journal of pain and symptom management 2000;20:193-201.

7. Cohen FL. Postsurgical pain relief: patients' status and nurses' medication choices. Pain 1980;9:265-74.

8. Dolin SJ, Cashman JN, Bland JM. Effectiveness of acute postoperative pain management: I. Evidence from published data. British journal of anaesthesia 2002;89:409-23.

9. Wheatley RG, Madej TH, Jackson IJ, Hunter D. The first year's experience of an acute pain service. British journal of anaesthesia 1991;67:353-9.

10. www.postoppain.org. Postoperative Pain Managment, 2007.

11. Idvall $E$, Hamrin $E$, Sjöström $B$, Unosson $M$. Quality indicators in postoperative pain management: a validation study. Scand J Caring Sci 2001;15:331-8.

12. Snyder $C$, Anderson $G$, Idvall $E$ et al. Do quality improvement organizations improve the quality of hospltal care for Medicare beneficiaries? Quality indicators in postoperative pain management: a validation study. JAMA 2005;293:2900-7.

13. Wit Rd. Postoperative pain: a point of vlew (in Dutch). Nederlands Tijdschrift voor Pijn en Pijnbestrijding 2005;24:33-4.

14. Rawal N. Acute pain services revisited--good from far, far from good? Regional anesthesia and pain medicine 2002;27:117-21.

15. Collins SL, Moore RA, McQuay HJ. The visual analogue pain intensity scale: what is moderate pain in millimetres? Pain 1997;72:95-7.

16. Jensen MP, Chen $C$, Brugger AM. Interpretation of visual analog scale ratings and change scores: a reanalysis of two clinical trials of postoperative pain. Journal of pain official journal of the American Pain Society 2003;4:407-14.

17. Owen $H$, McMillan V, Rogowski D. Postoperative pain therapy: a survey of patients' expectations and their experiences. Pain 1990;41:303-7.

18. Angster R, Hainsch-Mülter I. Postoperatives Schmerzmanagement. [Postoperative pain management] Anaesthesist 2005,54:505-31.

19. Fernandez E. A classification system of cognitive coping strategies for pain. Pain 1986;26:141-51.

20. Ong EL, Lim NL, Koay CK. Towards a pain-free venepuncture. Anaesthesia 2000;55:260-2.

21. Stamer UM, Mpasios N, Stuber F, Maier C. A survey of acute pain services in Germany and a discussion of international survey data. Regional anesthesia and pain medicine 2002;27:125-31.

22. Rosenquist RW, Rosenberg J. Postoperative pain guidelines. Regional anesthesia and pain medicine 2003;28:279-88.

23. Hannan EL, Magaziner $\mathrm{J}$, Wang $\mathrm{JJ}$ et al. Mortality and locomotion 6 months after hospitalization for hip fracture: risk factors and risk-adjusted hospital outcomes. JAMA 2001;285:2736-42

24. Poobalan AS, Bruce J, King PM et al. Chronic pain and quality of life following open inguinal hernia repair. $B r J$ Surg 2001;88:1122-6

25. Bruce J, Drury N, Poobalan AS et al. The prevalence of chronic chest and leg pain following cardiac surgery: a historical cohort study. Pain 2003;104:265-73.

26. Kalkman $\mathrm{CJ}$, Visser $\mathrm{K}$, Moen $\mathrm{J}$ et al. Preoperative prediction of severe postoperative pain. Pain 2003;105:415-23.

27. Pavlin DJ, Sullivan MJ, Freund PR, Roesen K. Catastrophizing: a risk factor for postsurgical pain. Clin $J$ Pain 2005;21:83-90. 


\section{Chapter 3}

\section{Predictors of acute postoperative pain after elective surgery}

Michael Sommer MD, Janneke M. de Rijke PhD, Maarten van Kleef MD PhD,

Alfons G.H. Kessels MD M.Sc., Madelon L. Peters PhD, José W.J.M. Geurts M.Sc, Jacob Patijn, Hans-Fritz Gramke MD, Marco A. E. Marcus MD PhD

Funding Support: The support came from the Pain Management and research centre subsidized by the Ministry of Health, the Netherlands

Accepted Clinical Journal of Pain

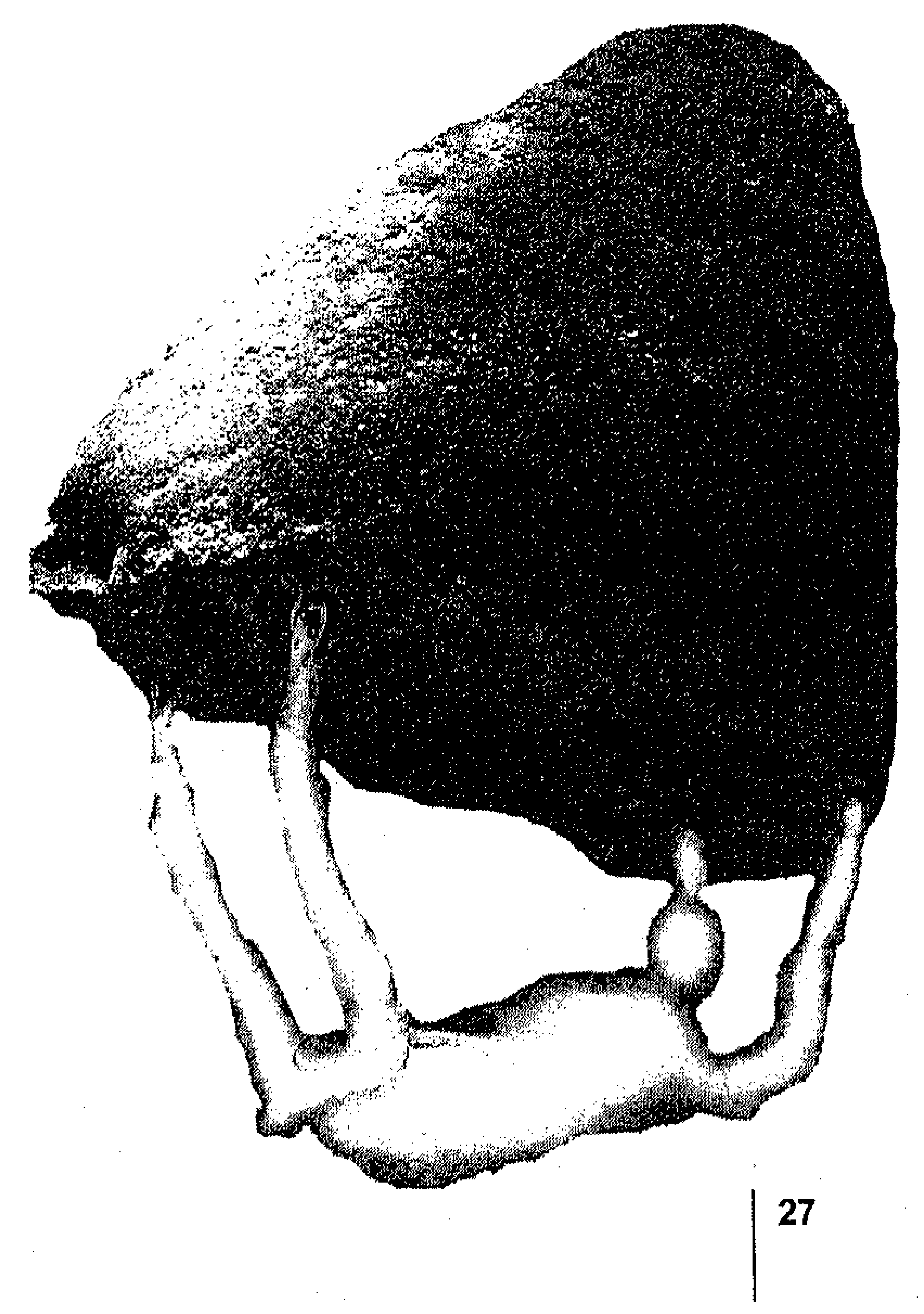




\section{CHAPTER}

\section{ABSTRACT}

Despite efforts to improve acute postoperative pain management a substantial number of patients still experience moderate to severe pain during the immediate postoperative days. The purpose of the present study was to identify predictors of moderate to severe acute postoperative pain. A total of 1490 patients undergoing heterogeneous surgical procedures recorded their pain three times a day on a $100 \mathrm{~mm}$ visual analogue scale from the day before the operation until 5 days post-operation. For each postoperative pain day, pain intensity was classified as "moderate" when the mean pain score was $41-74 \mathrm{~mm}$ and as "severe" when the means pain score was $75-$ $100 \mathrm{~mm}$. Using logistic regression analyses we examined the predictive value of a comprehensive set of pre- and peri- operative variables for moderate to severe pain. The most important predictors appeared to be preoperative pain, expected pain, surgical fear and pain catastrophizing.

Keywords:

Pain, Predictors, Prevalence, Postoperative, Surgery 


\section{INTRODUCTION}

Despite all research efforts to improve acute postoperative pain management still up to $30 \%$ of operated patients show pain scores higher than 3 on a Visual Analogue Scale (VAS) of $10(1-3)$. Adequate postoperative pain management is not only indispensable for reasons of humanity and patient's comfort, but there is a strong relation between effective postoperative pain management and the prevention of acute organ dysfunction $(4,5)$. Furthermore, high postoperative pain increases the risk of developing chronic pain $(5,6)$. Acute postoperative pain management is increasingly used as an indicator to judge the quality of intramural health care. The Joint Commission on Accreditation of Healthcare Organization (The Joint Commission) stated that the goal of acute postoperative pain management is to reduce patients' pain to a level VAS < 3 both at rest and during movement of the patient (7).

Few studies have tried to identify factors that could predict the seriousness of postoperative pain. In one study of 1416 postoperative patients, a relation between gender, age, preoperative pain, incision size, type of surgery on the one side and postoperative pain severity on the other side was identified (8). However, pain measurements in this study were restricted to the first three postoperative hours. In addition, the outcome measure in this study was defined as a numerical pain rating score above 8 of 10 at least once within the first hour after arrival on the Post Anaesthesia Care Unit (PACU), which is well above the VAS of 3 mentioned by The Joint Commission (7).

Other predictor studies in postoperative pain found an association between moderate or severe pain and age $(10,11)$ or female sex $(12)$. However in these studies the populations were limited to one or two surgical departments (12) or an observation period of 24 hours postoperatively (10).

Psychological factors have also been proposed to influence postoperative pain. An important determinant of acute postoperative pain intensity appears to be preoperative anxiety (13-15). However, because of the small number of predictor studies and the limitations of some of these studies, no definite conclusion can be drawn regarding factors predicting which patient is at risk of moderate or severe postoperative pain.

Therefore we conducted a prospective study to investigate the predictive value of a comprehensive set of both somatic and psychological factors on the level of postoperative pain.

\section{PATIENTS AND METHODS}

After approval of the institutional ethics committee and informed consent of the patients, consecutive patients admitted to the ward and scheduled for elective surgery at the University Hospital Maastricht, the Netherlands in the period February $1^{\text {st }}$ till August $30^{\text {th }} 2003$ were asked to participate in the study. The following surgical de- 


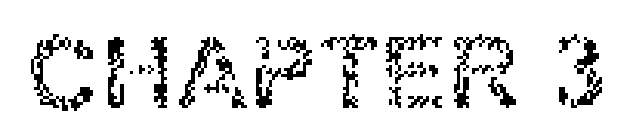

partments participated: general-, plastic-, ear-nose-throat-, facio-maxillary-, neurology- and thoracic surgery, orthopaedics, ophthalmology, gynaecology, and urology. Excluded were minors less than 18 years, patients with limitations of self-expression, visual dysfunction or Dutch language problems. Patients undergoing emergency surgery, cardiac surgery or caesarean section as well as patients receiving postoperative ventilatory support were also excluded from the study.

\section{Preoperative assessment, procedures and measurements}

Eligible patients received a letter describing the purpose and methods of the study and a set of questionnaires one to three weeks preceding the operation. The following questionnaires were included: 1. Pain catastrophizing scale (PCS; 13 items), measuring exaggerated negative interpretation of the meaning of pain $(16)(17) ; 2$. Behavioural inhibition scale (BIS; 7 items), measuring anxious temperament $(18,19)$; 3. Life Orientation Test (LOT; 8 items), measuring optimism (20) and General SelfEfficacy Scale (GSES) which measures the generalized belief that one has the skills to reach personal goals (10 items) $(21,22)$. Patients were requested to bring the completed questionnaires to the hospital on the day of admission.

After arrival on the surgical ward, a trained research assistant contacted the patients and explained the purpose and methods of the study. When the patient was willing to participate in the study, socio-demographic variables were recorded such as date of birth, sex and education and a 10-item questionnaire on surgical anxiety was filled out. ${ }^{\delta}$ Furthermore, pre-existing pain and expected pain after the operation were scored both using a $100 \mathrm{~mm}$ visual analogue scale (VAS) anchored to "no pain" being 0 and "worst pain I can imagine" being 100.

\section{Postoperative assessment and measurements}

Research assistants scored pain at rest using a Visual Analogue Scale on the day of operation (day 0 ) one and three hours postoperatively and at $9 \mathrm{pm}$. On days 1-4 after the operation, the patient scored pain using a pain diary, also three times a day. Research assistants visited all patients once a day to give assistance when necessary. When patients were physically not able to fill in the diary themselves, researchassistants offered assistance three times a day.

The type of anaesthesia was not regulated by the study protocol. The choice of technique and agents to be used was left to the individual anaesthesiologist and

\footnotetext{
$\delta$ 23. Koivula M, Tarkka MT, Tarkka $M$ et al. Fear and anxiety in patients at different time-points in the coronary artery bypass process. Int $\mathrm{J}$ Nurs Stud 2002;39:811-22.Originally the questionnaire was developed to measure fear of coronary bypass surgery.(22)For the present study, cardiac specific items and an item referring to death were removed. Factor analysis indicated two subscales: fear of immediate consequences of the operation (e.g. pain, anaesthetics) and fear of longer term consequences (inadequate recovery, financial consequences).
} 
patient. (Agents used for general anaesthesia were propofol, etomidate, sevoflurane and desflurane, sufentanil, fentanyl, rocuronium, and vecuronium). Perioperative pain was treated conformable the stepwise approach of acute pain treatment described by Rawal (24). The anaesthetic techniques used during the operation were documented as well as details of analgesia (epidural, spinal, intravenous, oral etc.) administered from 0 to 4 days post-surgery. After the operation all patients went to the PACU.

Patients who were discharged from hospital within four days postoperatively took their diary home and returned it to us in a special pre-paid envelope. Contacting patients by phone made it possible to retrieve diaries that had not been returned within 14 days after surgery.

All surgical procedures were categorized in three groups (minor, intermediate and major surgery) according to the anticipated level of postoperative pain (25). Subsequently, all surgical procedures were categorized according to the involved 7 anatomical body regions head/neck, upper extremities, thorax, back, upper and lower abdomen, and lower extremities (26) (Table 1).

According to our preoperative standardized protocol, all patients received paracetamol pre-emptively.

Postoperative pain in the minor operation group was treated with paracetamol (1000 mg) four times a day combined with no steroidal anti-inflammatory drugs (NSAIDs) administered by ward nurses. Escape medication in case of moderate or severe pain (VAS $>40$ ) was piritramide intra-muscularly.

Pain after intermediate operations was treated according to the same protocol combined with a small dose of intravenous piritramide $(2-5 \mathrm{mg})$ followed by intramuscular piritramide $10-15 \mathrm{mg} 6$ times a day.

For major operations piritramide was prescribed by intermittent intravenous infusion on patient's demand (PCA, Patient Controlled Analgesia), according to the protocol. The PCA protocol included three levels of piritramide doses $(1.0,1,5$ and $2 \mathrm{mg}$ per bolus, stop interval 5 minutes).

In many cases the major operations were conducted with a combination of general anaesthesia and epidural anaesthesia. Postoperatively, pain was treated by a continuous epidural infusion of bupivacaine $0.125 \%$ with sufentanil $0,5 \mu \mathrm{g} \mathrm{ml}$.

After a PACU observation period of 2-4 hours, patients with minor and intermediate operations returned to the surgical ward with nurses acquainted with the postoperative pain protocol. A trained postoperative pain nurse followed patients with major operations at least twice a day during four days postoperatively.

\section{Statistical analyses}

Mean pain VAS scores on the day of the operation and on postoperative days 0 to 4 (POD 1-4) were calculated, using the average of the three scores obtained from each individual on each of the days.

As the pain diaries were sometimes incomplete, totals could vary from day to day. A mean pain score of higher than $40 \mathrm{~mm}$ on a VAS was regarded as being unacceptable (27-29). 
CHAPTER 3

Table 1. Surgical procedures subdivided according to anticipated postoperative pain level and anatomical site.

\begin{tabular}{|c|c|c|}
\hline $\begin{array}{l}\text { Anticipated } \\
\text { pain level }\end{array}$ & Anatomical site & Surgical procedure \\
\hline \multirow[t]{6}{*}{ Minor } & $\begin{array}{l}\text { Head and neck } \\
\mathrm{n}=295\end{array}$ & $\begin{array}{l}\text { Thyroidectomy, stapedectomy, tympanoplasty, petrosal bone } \\
\text { and middle ear surgery, cochlear implantation, middle ear } \\
\text { inspection, middle ear reconstruction, auricle reconstruction, } \\
\text { nasal sinus inspection, endoscopy of pharynx, larynx, bronchus, } \\
\text { intracranial tumour surgery, craniotomy, eye surgery }\end{array}$ \\
\hline & $\begin{array}{l}\text { Upper extremity } \\
n=10\end{array}$ & Peripheral vascular operations, wound toilet, sutures, \\
\hline & $\begin{array}{l}\text { Thorax- noncardiac } \\
n=10\end{array}$ & Vascular operations, wound toilet, \\
\hline & $\begin{array}{l}\text { Lower abdomen/pelvis } \\
n=138\end{array}$ & $\begin{array}{l}\text { Vaginal urological procedures, cervix operation, abortion, hys- } \\
\text { teroscopy, operations of male genital, endoscopic urological } \\
\text { interventions e.g. TUR, cystic biopsy, urethra, cystoscopy, } \\
\text { plastic skin operations }\end{array}$ \\
\hline & $\begin{array}{l}\text { Lower extremity } \\
n=20\end{array}$ & $\begin{array}{l}\text { plastic skin operations, peripheral vascular operations, wound } \\
\text { toilet }\end{array}$ \\
\hline & $\begin{array}{l}\text { Back/Spinal } \\
n=13\end{array}$ & Urological neuromodulation, small plastic skin operations, \\
\hline \multirow[t]{8}{*}{ Intermediate } & $\begin{array}{l}\text { Head and neck } \\
n=85\end{array}$ & $\begin{array}{l}\text { Neck dissection, mouth and throat surgery, laryngectomy, } \\
\text { pharynx and larynx surgery, maxilla and mandibular surgery }\end{array}$ \\
\hline & $\begin{array}{l}\text { Upper extremity } \\
n=39\end{array}$ & Orthopaedic hand and arm surgery, \\
\hline & $\begin{array}{l}\text { Thorax- noncardiac } \\
n=31\end{array}$ & Oncological and plastic breast surgery \\
\hline & $\begin{array}{l}\text { Upper abdomen } \\
\mathrm{n}=69\end{array}$ & $\begin{array}{l}\text { Fundoplication, duodenum surgery, cholecystectomy, nephrec- } \\
\text { tomy, }\end{array}$ \\
\hline & $\begin{array}{l}\text { Lower abdomen/pelvis } \\
n=195\end{array}$ & $\begin{array}{l}\text { Surgery of vulva, ovary, adnexen, vaginal and abdominal uter- } \\
\text { usextirpation, abd. endometriosis, closure of anus praeter, } \\
\text { colorectal surgery, anal surgery, abdominal vascular surgery, } \\
\text { abd. lipectomy, cystic resection, Ryntshak and radical prostatec- } \\
\text { tomy }\end{array}$ \\
\hline & $\begin{array}{l}\text { Lower extremity } \\
n=212\end{array}$ & $\begin{array}{l}\text { Plastic skin operations, orthopaedic surgery foot/knee/leg/hip, } \\
\text { amputation, peripheral vascular surgery }\end{array}$ \\
\hline & $\begin{array}{l}\text { Back/spinal } \\
n=63\end{array}$ & $\begin{array}{l}\text { Plastic skin operations, spinal cord decompression with hemi- } \\
\text { laminectomy, discectomy }\end{array}$ \\
\hline & $\begin{array}{l}>\text { than } 1 \text { site } \\
\mathrm{n}=12\end{array}$ & Plastic breast + abdominal surgery, arm/hand and hip (fractures) \\
\hline \multirow[t]{5}{*}{ Major } & $\begin{array}{l}\text { Upper extremity } \\
n=23\end{array}$ & Shoulder surgery (endoprothesis), clavicle surgery \\
\hline & $\begin{array}{l}\text { Thorax- noncardiac } \\
n=10\end{array}$ & Lobectomy \\
\hline & $\begin{array}{l}\text { Upper + lower abdomen } \\
n=171\end{array}$ & $\begin{array}{l}\text { Hepatectomy, pancreatectomy, combination of different bowel } \\
\text { surgery, aortic surgery, extensive gynaecological surgery, } \\
\text { combination of bowel and gynaecological surgery }\end{array}$ \\
\hline & $\begin{array}{l}\text { Lower extremity } \\
n=60\end{array}$ & Total knee replacement \\
\hline & $\begin{array}{l}\text { Back/spinal } \\
n=34\end{array}$ & Spondylodesis, spinal cord tumour resection, untethering \\
\hline
\end{tabular}


Missing values in predictor variables were imputed. This was done because of a presumed non-response problem where the occurrence of missing data is related to the outcome value pain. Our hypothesis was that patients, who didn't fill in all data, presumably had severe pain on that moment or no pain at all. Either way this could influence our outcome. We imputed missing values of predictor values according to the multiple imputation (MI) method described by van Buuren and Rubin.(30) In MI each missing value is replaced by a set of $M>1$ plausible values drawn from their predictive distribution. We performed $M I$ with $M=5$, obtaining 5 complete datasets, with imputed values for short term fear ( $n$ missing $=37 ; 2.5 \%)$, long term fear $(n=36$; $2.4 \%)$, pain catastrophizing $(n=51 ; 3.4 \%)$, expected pain $(n=51 ; 3.4 \%)$, preoperative pain $(n=8 ; 0.5 \%)$, optimism $(n=55 ; 3,7 \%)$, self efficacy $(n=94,6.3 \%)$ and anxiety disposition ( $n=94 ; 6.3 \%$ ). On each of the 5 complete datasets stepwise multiple logistic regression analysis was performed to identify the factors that independently predicted the risk of having postoperative pain $>40$ on days 0 to 4 after surgery. Easily obtainable predictors were included first (table 3 ). In the first block two variables related to the surgical procedure were entered: type of surgery (minor, intermediate, major) and anatomical body region. In the second block the demographic variables age (three groups: $<45$ years, $45-59$ years and 60 years and older ) and sex were entered. Preoperative pain (yes or no VAS $>40$ ), duration of the operation (yes or no $\geq 3$ hours), anaesthetic technique (general, loco-regional or general + loco-regional), and ASA grade were entered in the third block using a forward stepwise procedure while the psychological parameters (expected pain, pain catastrophizing, self-efficacy, anxiety disposition, surgical anxiety and optimism) were examined in the final step, also with a forward stepwise procedure. Psychological variables were dichotomized by median split. In all steps a p-value of 0.05 was used for keeping variables in the model. Next, the results (estimates of coefficients and standard errors) of the identical analyses on each of the 5 datasets were combined to calculate overall estimates, standard errors and $95 \%$ confidence intervals. $(30,31)$ Predictors were included in the final model if they were significant predictors in more than three datasets. The models ability to discriminate between patients with and without unacceptable postoperative pain was estimated by the area under the receiver operant curve (AUC) for the successive blocks.

Missing values in outcome measures (pain scores) were not imputed. However, this missing data could also be subject to selection processes. Therefore we investigated with logistic regression analyses whether these missing outcomes could be predicted with available covariates (age, type of operation, sex etc.). The results revealed some significant relationships and were used to calculate a weight factor for each case.

Analyses were performed with STATA (version 8) and SPSS (version 12). 


\section{RESULTS}

Data on a total of 1490 patients were studied. Patient characteristics are presented in table 2. Slightly fewer male patients participated in this study than female patients ( $47 \%$ versus $53 \%$ ). Forty-five per cent of the patients were 60 years of age or older. $1663(85 \%)$ of 1975 eligible subjects consented to participate. 140 (8\%) patients dropped out for a variety of reasons: postoperative ventilatory support $(n=50)$, too burdensome for the patient $(n=32)$, complications followed by a new operation $(n=14)$, and other diverse reasons $(n=44)$. From $33(2 \%)$ patients the questionnaires were not assessable because too many data were missing, leaving 1490 evaluated patients. Distribution of age and gender among the non-responders was not significantly different from the participating patients.

We performed a logistic regression analysis for the risk of moderate or severe postoperative pain (i.e. pain $>40$ on a $100 \mathrm{~mm}$ VAS) on the day of operation and day one to four (table 3 ).

If the surgical procedure was categorized as intermediate with respect to the anticipated postoperative pain the risk of higher VAS was increased on day 0 and 1. If the operation was categorized as being major the risk was increased on day 1 and 2.

With respect to the anatomical site the head and neck procedures functioned as reference $(=1.0)$. The risk of pain $>40$ on a $100 \mathrm{~mm}$ VAS was statistically higher after upper-extremity-operations on day one but not on the other days. After leg or hip surgery the risk of moderate or severe pain was higher on the operation day.

Age as a risk factor played only a role in the group 45 - 59 versus 60 years on day 0 and 3 . The patients of the former group had a significant higher risk of pain $>$ $40 \mathrm{~mm}$ on those days. Female sex protected against pain on day 0.

Pre-existing pain increased the risk of postoperative pain significantly on all days. The anaesthetic technique played a role when general anaesthesia was compared to general plus regional anaesthesia or regional anaesthesia alone. The Odd's ratio indicates that the risk of pain $>40 \mathrm{~mm}$ is significantly higher in patients receiving general anaesthesia only immediately postoperative and on POD 2.

\section{Psychological predictors}

Expected pain $>40 \mathrm{~mm}$ increased the risk of postoperative pain significantly on all postoperative days. A higher short term fear score (definition in (6)) increased the risk of pain $>40 \mathrm{~mm}$ on day 0 , a higher long term fear score (definition in (6)) increased the risk of pain on day 1 to 3 . If a patient scored high on the pain catastrophizing scale beforehand he had a significantly higher risk of having pain $>40 \mathrm{~mm}$ afterwards on day 2 - 4 .

Generalized self-efficacy seemed to decrease the risk of postoperative [ain, however, this only reached significance on POD 4. Unexpectedly, we also found anxious temperament to decrease the risk of postoperative pain, but this only reached significance on the day of the operation. 
PREDICTORS OF POSTOPERATIVE PAIN

Table 2. Total patient characteristics and according to the number $(n)$ and proportion $(\%)$ of patients with a mean pain in rest of $>$ than 40 on a visual analogue scale (VAS)

\begin{tabular}{|c|c|c|c|c|c|c|}
\hline & \multirow[t]{2}{*}{ Total } & \multicolumn{5}{|c|}{ Mean VAS $>40$} \\
\hline & & $\begin{array}{l}\text { Operation } \\
\text { day }\end{array}$ & POD 1 & POD 2 & POD 3 & POD 4 \\
\hline & $n=1490$ & $n=1398$ & $n=1300$ & $n=1295$ & $n=1275$ & $n=1247$ \\
\hline & $\mathrm{N} / \%$ & $N / \%$ & $N / \%$ & $N / \%$ & $N / \%$ & $N / \%$ \\
\hline \multicolumn{7}{|l|}{ Age } \\
\hline$<45$ years & $374 / 25$ & $151 / 42$ & $112 / 35$ & $66 / 21$ & $47 / 15$ & $49 / 16$ \\
\hline $45-59$ years & $449 / 30$ & $179 / 43$ & $134 / 33$ & $78 / 19$ & $80 / 20$ & $59 / 15$ \\
\hline $60+$ years & $667 / 45$ & $182 / 29$ & $147 / 25$ & $97 / 17$ & $75 / 13$ & $59 / 11$ \\
\hline mean $(y r)(s d)$ & $56(15.5)$ & & & & & \\
\hline \multicolumn{7}{|l|}{ Sex } \\
\hline Male & $702 / 47$ & $193 / 30$ & $145 / 24$ & $93 / 15$ & $78 / 13$ & $62 / 11$ \\
\hline Female & $788 / 53$ & $319 / 43$ & $248 / 36$ & $148 / 22$ & $124 / 19$ & $105 / 16$ \\
\hline \multicolumn{7}{|c|}{ Type of Anaesthesia } \\
\hline General & $1024 / 69$ & $402 / 42$ & $269 / 30$ & $175 / 20$ & $141 / 16$ & $113 / 13$ \\
\hline Loco- regional ${ }^{1}$ & $270 / 18$ & $50 / 28$ & $58 / 25$ & $33 / 14$ & $31 / 14$ & $21 / 10$ \\
\hline $\begin{array}{l}\text { General + } \\
\text { Locoregional }^{2}\end{array}$ & $196 / 13$ & $60 / 23$ & $66 / 38$ & $33 / 19$ & $30 / 17$ & $33 / 20$ \\
\hline \multicolumn{7}{|l|}{ Type of surgery } \\
\hline Minor & $486 / 33$ & $105 / 23$ & $71 / 16$ & $51 / 12$ & $39 / 9$ & $36 / 9$ \\
\hline Intermediate & $706 / 47$ & $295 / 44$ & $212 / 35$ & $122 / 20$ & $104 / 17$ & $75 / 13$ \\
\hline Major & $298 / 20$ & $112 / 42$ & $110 / 43$ & $68 / 27$ & $59 / 24$ & $56 / 23$ \\
\hline \multicolumn{7}{|l|}{ ASA -score } \\
\hline 1 & $538 / 36$ & $206 / 40$ & $145 / 31$ & $82 / 17$ & $72 / 15$ & $57 / 13$ \\
\hline 2 & $684 / 46$ & $221 / 35$ & $186 / 30$ & $117 / 19$ & $91 / 15$ & $76 / 13$ \\
\hline $3-4$ & $268 / 18$ & $85 / 36$ & $62 / 29$ & $42 / 19$ & $39 / 18$ & $34 / 17$ \\
\hline \multicolumn{7}{|c|}{ Duration of operation } \\
\hline$<3$ hours & $1045 / 70$ & $240 / 27$ & $243 / 26$ & $143 / 16$ & $122 / 13$ & $104 / 12$ \\
\hline 3 hours or more & $445 / 30$ & $150 / 39$ & $150 / 39$ & $98 / 25$ & $80 / 21$ & $63 / 16$ \\
\hline Total & $1490 / 100$ & $512 / 34$ & $393 / 30$ & $241 / 19$ & $202 / 16$ & $167 / 14$ \\
\hline
\end{tabular}

${ }^{1} 40$ patients in this group received 'peripheral blocks'; ${ }^{2}$ Five patients in this group received general anaesthesia + 'peripheral block' 
CMAPTER 3

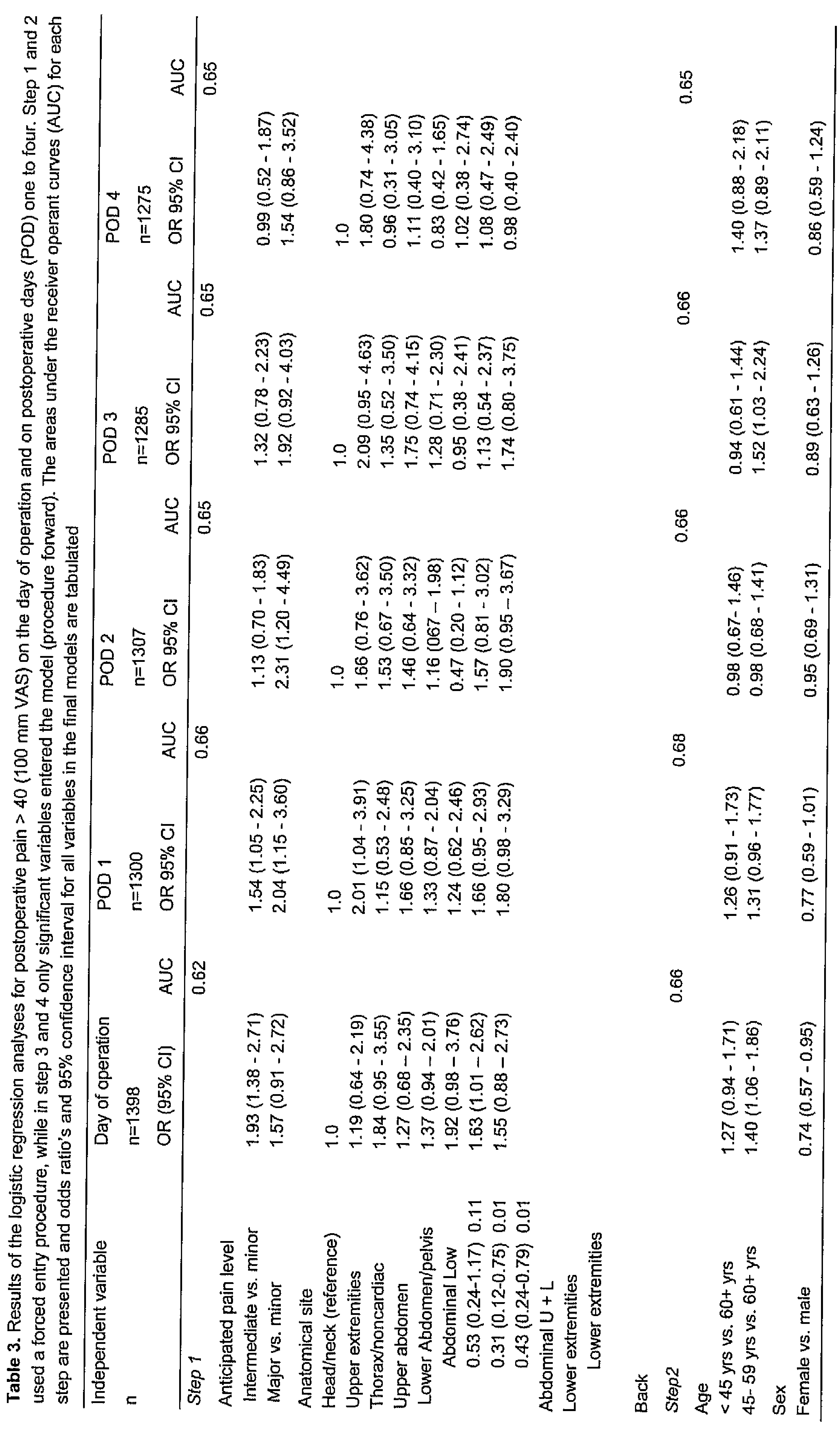


PREDICTORS OF POSTOPERATVE PAN

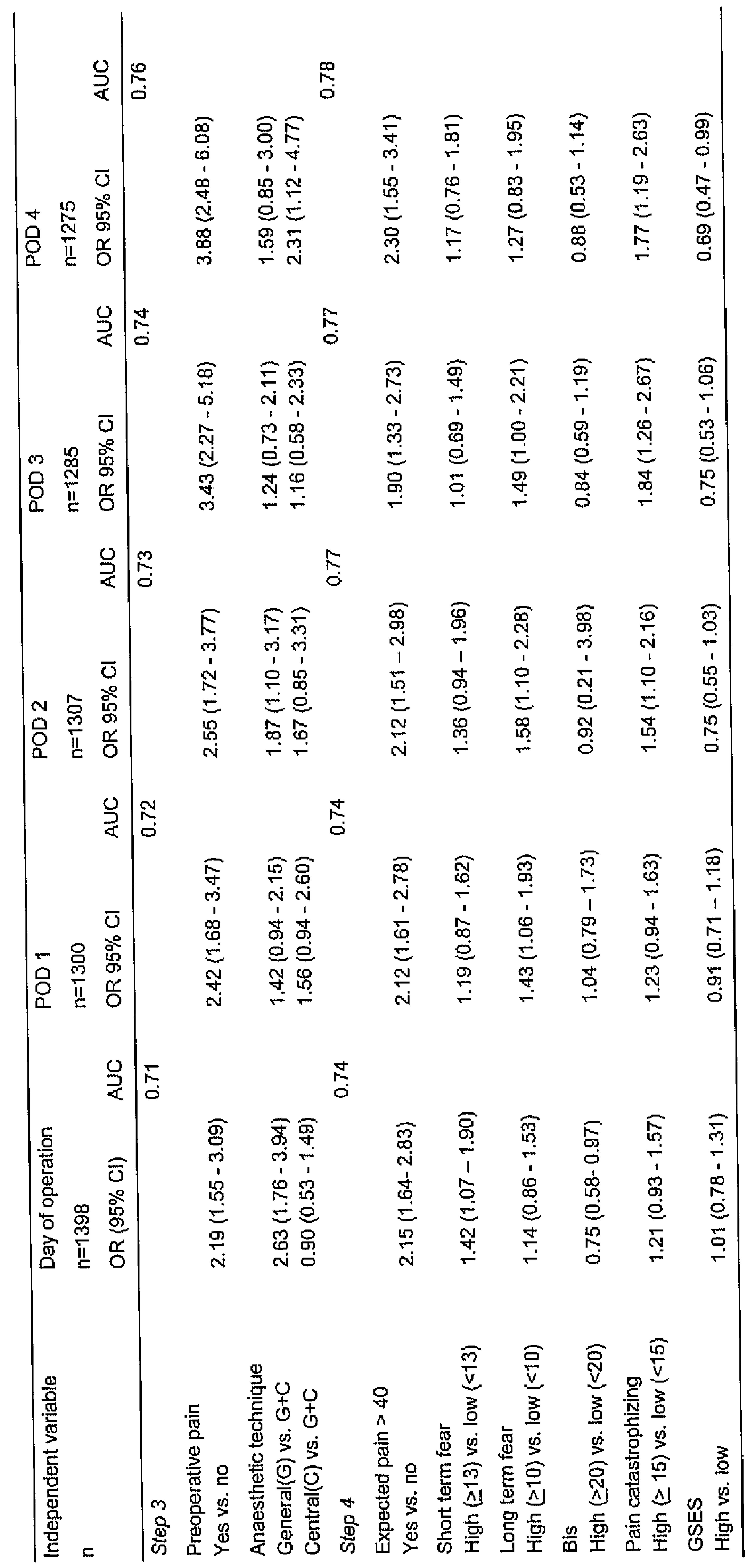




\section{DISCUSSION}

As far as we know no other study about acute pain predictors has investigated demographical, somatically and psychological items in so many patients. We followed a total of 1490 patients undergoing various types of surgical interventions one day prior to and four days after the operation to find predictors of immediate postoperative pain.

The strongest predictors of moderate or intense postoperative pain in our study were pre-existing pain and expected pain $>40$ on a $100 \mathrm{~mm}$ VAS. Both parameters were statistically significant on all 5 days. Other predictors were Pain Catastrophizing that was statistically significant from day 2 until the end of our study and Long Term Fear which was statistically significant on day 1 to 3 . All other items either showed statistical significance incidentally but not during a longer period of time or showed no significance at all.

The age group $45-60$ years had significantly more postoperative pain on the day of operation and on POD 3 compared to the group $65+$. In other studies younger age was found to be correlated with more postoperative pain. Macintyre and Jarvis analyzed retrospectively data of 1000 patients in the first 24 hours postoperatively after different kind of surgery (10). Patients $15-39$ years of age had more pain than patients $61-69$ years old. The authors argued that pharmacokinetic and pharmacodynamic factors play a role in a different analgesic demand of the different age groups. Decrease in volume distribution and clearance of morphine and decrease in plasma albumin would be responsible for diminished morphine requirements in older people. However, this study used retrospective analysis of the first 24 hours of the postoperative period. In our data it is the middle-aged group of patients who have higher pain scores. Patients older than 65 years and younger than 40 years have less pain than the group 41 to 64 years of age. Pharmacokinetics can hardly play a role in the middle-aged group when on the other hand the youngest patients and the $65+$ group had comparable VAS. One would expect the $40-59$ years old to have the same volume distribution, morphine clearance and plasma albumin levels as the group $18-39$ years old. A study measuring plasma levels of analgesics in different age groups could answer this pharmacokinetic question.

Another indicator was "pain before the operation". We found that the risk of moderate or severe pain after surgery increased significantly if patients suffered from pain before the operation compared to those who did not have pre-existing pain. This is conform the literature $(8,11)$. The basic principal of preoperative pain to influence postoperative pain is not clear. A possible mechanism could be neuroplastic changes in the spinal cord due to chronic noxious input (8). However, this is a hypothesis that is difficult to prove.

Together with "expected pain" these two items were strong predictors of increased postoperative pain on all 5 days. 


\section{Factors due to the operation}

After upper extremity operations there is an increased risk of postoperative pain on day one. Most of the upper extremity procedures got single shot regional anaesthesia with or without general anaesthesia. After 12 hours or less the local aesthetics are metabolized and pain arises often between 2 and 4 o'clock am. Most of these procedures in our hospital are performed in a 24 hours setting: the patient will leave the hospital the morning after the operation. A continuous technique is therefore not preferable as complete neurological function at discharge is required. Probably systemic analgesics were started too late to prevent pain.

After hip- or leg-surgery the risk of moderate or severe pain was increased on day 0 . Most of these procedures in our hospital are performed under spinal anaesthesia. Probably, patients went back to the ward before analgesic treatment was well adjusted and sensory motor function was restored completely.

\section{Psychological parameters}

Expected pain above 40 and pain catastrophizing added to the predictive power of the model. Pain catastrophizing turned up as risk factor for postoperative pain in other studies as well. Pavlin et al examined 48 patients with anterior cruciate ligament repair and found that when PCS was above 13 the postoperative VAS was 33 to $74 \%$ higher and duration of moderate to severe pain was more prolonged (32). In this study, a high score on the PCS was associated with an increased risk of pain postoperatively from the second day post-operation until the fourth (and last) day of observation.

Patients with a higher long-term fear score had an increased risk of more postoperative pain on POD 1 to 3 . In an earlier publication about long-term consequences of postoperative pain we found that a higher long-term fear score was associated with more pain on a 6 month follow up as well (6). This is in line with other studies on acute postoperative pain and recovery where fear of surgery proved to be the most consistent psychological predictor of unfavourable outcome $(8,14)$. Kalkman found more pain in the early postoperative period if the preoperative anxiety score was high. We found short term fear to be associated with more postoperative pain on the first day but on the other days it was long term fear.

In summary these are data of a large cohort with only few missing data, and a 4 day follow-up to predict which patients are at risk for acute severe postoperative pain. As strong predictors of postoperative pain after surgery we found: preoperative pain, expected pain above 40 on a $100 \mathrm{~mm}$ VAS, pain catastrophizing, and long term fear. 


\section{CHAPTER}

\section{REFERENCES}

1. Botti M, Bucknall T, Manias $E$. The problem of postoperative pain: issues for future research. Int $J$ Nurs Pract 2004;10:257-63.

2. Sauaia A, Min SJ, Leber $\mathrm{C}$ et al. Postoperative pain management in elderly patients: correlation between adherence to treatment guidelines and patient satisfaction. J Am Geriatr Soc 2005;53:274-82.

3. Warfield $\mathrm{CA}, \mathrm{Kahn} \mathrm{CH}$. Acute pain management. Programs in U.S. hospitals and experiences and attitudes among U.S. adults. Anesthesiology 1995;83:1090-4.

4. Joshi GP, Ogunnaike BO. Consequences of inadequate postoperative pain relief and chronic persistent postoperative pain. Anesthesiol Clin North America 2005;23:21-36.

5. Gagliese L, Katz J. Age differences in postoperative pain are scale dependent: a comparison of measures of pain intensity and quality in younger and older surgical patients. Pain 2003;103:11-20.

6. Peters ML, Sommer M, de Rijke JM et al. Somatic and psychologic predictors of long-term unfavorable outcome after surgical intervention. Ann Surg 2007;245:487-94.

7. Perkins FM, Kehlet $\mathrm{H}$. Chronic pain as an outcome of surgery. A review of predictive factors. Anesthesiology 2000;93:1123-33.

8. Kalkman $\mathrm{CJ}$, Visser $\mathrm{K}$, Moen $\mathrm{J}$ et al. Preoperative prediction of severe postoperative pain. Pain 2003;105:415-23

10. Macintyre PE, Jarvis DA. Age is the best predictor of postoperative morphine requirements. Pain 1996;64:357-64.

11. Thomas $T$, Robinson $C$, Champion $D$ et al. Prediction and assessment of the severity of postoperative pain and of satisfaction with management. Pain 1998;75:177-85.

12. Uchiyama $K$, Kawai $M$, Tani $M$ et al. Gender differences in postoperative pain after laparoscopic cholecystectomy. Surg Endosc 2006;20:448-51.

13. Croog SH, Baume RM, Nalbandian J. Pre-surgery psychological characteristics, pain response, and activities impairment in female patients with repeated periodontal surgery. J Psychosom Res 1995;39:3951.

14. Munafo MR, Stevenson J. Anxiety and surgical recovery. Reinterpreting the literature. J Psychosom Res $2001 ; 51: 589-96$.

15. Caumo W, Schmidt AP, Schneider $\mathrm{CN}$ et al. Preoperative predictors of moderate to intense acute postoperative pain in patients undergoing abdominal surgery. Acta Anaesthesiol Scand 2002;46:1265-71.

16. Sullivan M, Bishop S, Pivik J. The Pain Catastrophizing Scale: development and validation. 1995;7:52432.

17. Van Damme S, Crombez G, Bijttebier $P$ et al. A confirmatory factor analysis of the Pain Catastrophizing Scale: Invariant factor structure across clinical and non-clinical populations. Pain 2002;96;319-24.

18. Carver CS, White TL. Behavioral inhibition, behavioral activation, and affective responses to impending reward and punishment: The BIS/BAS Scales, Journal of Personality and Social Psychology 1994;67:319-33.

19. Carver CS, Pozo-Kaderman C, Harris SD et al. Optimism versus pessimism predicts the quality of women's adjustment to early stage breast cancer. Cancer 1994;73:1213-20.

20. Scheier MF, Carver CS, Bridges MW. Distinguishing optimism from neuroticism (and trait anxiety, selfmastery, and self-esteem): a reevaluation of the Life Orientation Test. J Pers Soc Psychol 1994;67:106378.

21. Torres A, Enk CD, Hochberg M, Srebnik M. Porphyra-334, a potential natural source for UVA protective sunscreens. Photochem Photobiol Sci 2006;5:432-5.

22. Jerusalem M S. Self-efficacy as a resource factor in stress apparaisal processes. In: Schwarzer R, ed. Self-effcacy: Thought control of action. Washington DC, USA: Hemisphere. 1994:195-213.

23. Koivula M, Tarkka MT, Tarkka $M$ et al. Fear and anxiety in patients at different time-points in the coronary artery bypass process. Int $J$ Nurs Stud 2002;39:811-22.

24. Rawal N. Postoperative pain and its management. In: Rawal N, ed. Management of acute and chronic pain London: BMJ Books, 1989.

25. Sommer $M$, de Rijke JM, van Kleef $M$ et al. The prevalence of postoperative pain in a sample of 1490 surgical inpatients. Eur $\mathrm{J}$ Anaesthesiol 2007:1-8.

26. Rawal N. Acute pain services revisited--good from far, far from good? Regional anesthesia and pain medicine 2002;27:117-21. 


\section{PWEDICTORS OF POSTOPERATVEPAN}

27. Collins SL, Moore RA, McQuay HJ. The visual analogue pain intensity scale: what is moderate pain in millimetres? Pain 1997;72:95-7.

28. Dolin SJ, Cashman JN, Bland JM. Effectiveness of acute postoperative pain management: I. Evidence from published data. British journal of anaesthesia 2002;89:409-23.

29. Jensen MP, Chen $C$, Brugger AM. Interpretation of visual analog scale ratings and change scores: a reanalysis of two clinical trials of postoperative pain. J Pain 2003;4:407-14.

30. van Buuren S, Boshuizen HC, Knook DL. Multiple imputation of missing blood pressure covariates in survival analysis. Stat Med 1999;18:681-94.

31. Zhou YB, Jiang QW, Zhao GM. [A method to manage of miss data--multiple imputation]. Zhonghua $Y u$ Fang Yi Xue Za Zhi 2004;38:424-6.

32. Pavlin DJ, Sullivan MJ, Freund PR, Roesen K. Catastrophizing: a risk factor for postsurgical pain. Clin J Pain 2005;21:83-90. 


\section{Chapter 4}

\section{Prevalence and predictors of postoperative pain after ear, nose and throat (ENT) surgery}

Michael Sommer MD, José W.J.M. Geurts MSc, Bjorn Stessel MD, Alfons G.H. Kessels MD MSc, Madelon L. Peters PhD, Jacob Patijn MD PhD, Maarten van Kleef MD PhD, Bernd Kremer MD PhD, Marco A. E. Marcus MD PhD

Funding Support: The support came from the Pain Management and Research Center subsidized by the Ministry of Health, The Netherlands Accepted Archives of Otolaryngology and Head \& Neck Surgery.

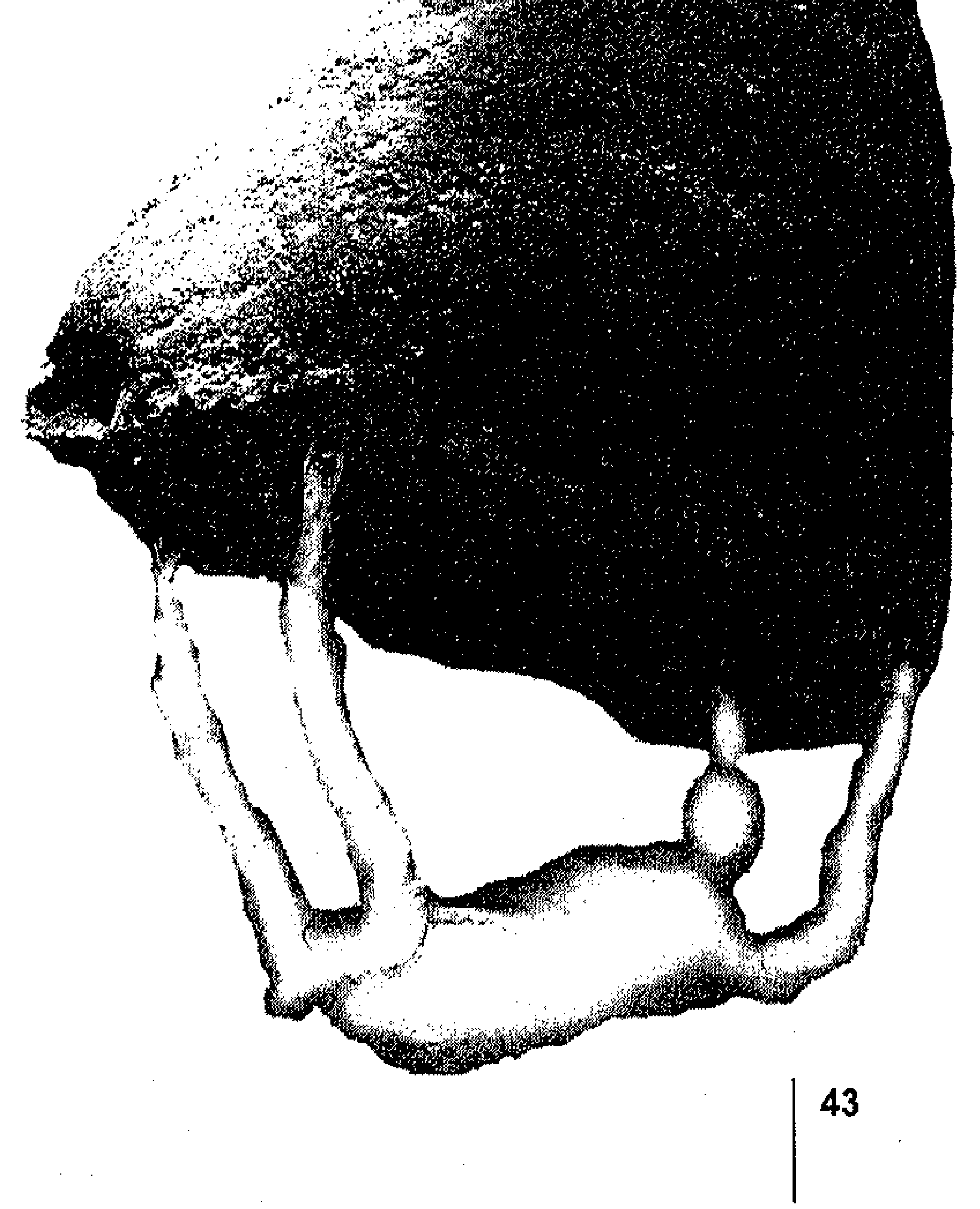




\section{CHAPTER A}

\section{ABSTRACT}

Introduction: There is little data in the literature on postoperative pain in different types of ear, nose and throat (ENT) surgery and their psychological preoperative predictors.

Materials and methods: 217 ENT-surgery patients between the age of 18 and 80 consented to participate in a study to investigate the presence of unacceptable postoperative pain (Visual Analogue Scale (VAS)>40). Five subgroups according to anatomical site of operation were made. Unacceptable pain was calculated (a mean of three scores per day, day 1-4). Fourteen candidate predictors for unacceptable postoperative pain were selected (age, gender, duration of surgery, American Society of Anesthesiology (ASA) score, preoperative pain, expected pain, fear, catastrophizing, life orientation). Bivariate analysis for candidate predictor and anatomical site was performed. Multivariate logistic regression analysis was used on all predictors with a p-value of $<0.15$ in the bivariate analysis to derive independent predictors.

Results: $50 \%$ of the patients undergoing surgery on the (oral, pharynx and larynx)region and on the (neck and salivary gland)-region had a VAS score $>40$ on day one. In the first group, VAS remained high on all 4 days. VAS $>40$ after endoscopic procedures $<30 \%$, and after ear- and nose surgery $<20 \%$.

After bivariate analysis, six variables: age, gender, preoperative pain, expected pain, short-term fear and pain catastrophizing had a predictive value. Multivariate analysis showed only preoperative pain, pain catastrophizing and anatomical site of operation as independent predictors.

Conclusions: First, differences exist in prevalence of unacceptable postoperative pain between ENT-operations on different anatomical sites.

Second, a limited set of variables can be used to predict the occurrence of unacceptable postoperative pain after ENT-surgery. 


\section{INTRODUCTION}

Adequate postoperative pain management is an essential part of peri-operative care, as postoperative pain results in patient discomfort and may decrease patient satisfaction (1). More importantly, it may increase the risk for pulmonary and cardiovascular complications and may even contribute to the development of chronic pain (2-4). Although in the last few decades a vast range of new therapeutic developments have occurred, for example in new formulations of pain medication, postoperative pain remains a persistent problem $(5,6)$. To improve postoperative pain control, it would be desirable to distinguish patients and patient groups preoperatively who are at risk of developing unacceptable high levels of postoperative pain. Type of surgery is a factor determining the level of postoperative pain (7). However, with the exception of pain after tonsillectomy, a common and very painful procedure, surprisingly little literature is available on the amount of postoperative pain after Ear, Nose and Throat surgery (ENT-surgery) $(8,9)$. Absence from work during more than two weeks, following routine ENT-surgery and more than 10 percent readmission or overstay after day case nasal surgery suggests that this patient group cannot be neglected, and pain in ENT patients is probably highly underestimated $(10,11)$.

Type of surgery is not the only determinant of postoperative pain since patients undergoing the same procedure may require plasma levels of opiates varying more than 5 -fold to provide satisfying analgesia. Other determinants (determined in groups that consisted of all types of surgery) that have been suggested to predict postoperative pain are female gender, younger age, the amount of preoperative pain and psychological factors including preoperative anxiety and catastrophizing (12-17).

The purpose of this study was to objectify postoperative pain in different types of ENT-surgery, using a visual analogue scale (VAS), and to identify somatic and psychological preoperative predictors for unacceptable high levels of pain in this patient group. A mean pain score of higher than $40 \mathrm{~mm}$ on a VAS is generally regarded as being unacceptable (18-20). Patients undergoing ENT-surgical procedures participated in this study that consisted of preoperative assessments of somatic and psychological variables and daily assessment of pain until day four postoperatively.

\section{METHODS}

\section{Design}

This study is a secondary analysis of data from two prevalence studies, with a longitudinal design, that were carried out to obtain short-term follow-up data on surgery related pain on days $0-4$ postoperatively (21). In the two surveys data was obtained from 2138 consecutive patients, 304 patients had been admitted for Ear Nose Throat (ENT) surgery. The final study sample comprised 217 ENT patients. From these 217 subjects 181 were admitted to a surgical ward and 36 to a day-case unit. The charac- 


\section{CWAPTER 4}

teristics of the patients who refused were no different in demographics and type of operation from those of the participants. Data on a total of 217 patients were studied.

\section{Subjects}

This study was done in a general university teaching hospital with 715 beds, 183 beds are located on surgical wards. The institutional Ethics Committee approved the survey.

All patients between the age of 18 and 80 scheduled for ENT surgery were approached for possible study participation from October 2002 until September 2003. From October 2002 until January 2003 the patients scheduled for ENT surgery in a same day admission unit were approached and from January 2003 until September 2003 ENT surgery inpatients were enrolled. Each subject was evaluated for eligibility to participate in the study (over the age of 18; no limitations of self-expression; no visual dysfunction or language problems). Patients admitted for acute surgery or requiring postoperative ventilatory support were excluded from the study. Excluded were 82 patients; 36 patients were not meeting inclusion criteria, 17 refused to participate (less than $1 \%$ ), 29 patients did not participate for other reasons. The characteristics of the patients who refused were no different in demographics and type of operation from those of the participants. Data on a total of 217 patients were studied.

\section{Outcome and candidate predictors}

The outcome of the present study was the presence of unacceptable postoperative pain, defined as a mean pain score of $40 \mathrm{~mm}$ or more on a visual analogue scale (VAS) (18-20). The pain score is a mean score of three scores during the day of surgery and day $1-4$ postoperatively.

Fourteen candidate predictors of unacceptable postoperative pain were selected. These included somatic variables; type of operation, age, gender, duration of surgery, ASA score, preoperative pain and type of admission and psychological variables; expected pain, surgical fear, catastrophizing and optimism. ASA score describes the patient from the perspectives of basic risk banding. Research into perioperative outcome use these scores widely as descriptors of the surgical population

\section{Instruments and procedures}

The data collection instruments consisted of preoperative questionnaires and a postoperative pain and medication diary.

\section{Preoperative assessment}

Eligible patients received a letter describing the purpose and methods of the study and a set of questionnaires one to three weeks preceding treatment. The following questionnaires were included. $\{1\}$ Pain catastrophizing scale (PCS;13 items), measuring an exaggerated negative attitude towards pain and $\{2\}$ Life Orientation Test 
(LOT; 8 items), which measures dispositional optimism $(14,16)$. Completion of questionnaires took 7-10 minuten. Patients were requested to bring the completed questionnaires to the hospital on the day of admission.

After admission to the surgical ward or day case unit, a trained research assistant explained the purpose and methods of the study to each eligible patient who was willing to participate. Socio-demographic variables were recorded such as date of birth, sex and education and a questionnaire on surgical fear was filled out. This $10-$ item questionnaire on surgical fear was adapted from Koivola et al (22). For the present study, only the subscale "fear of immediate consequences" ( 4 items, Cronbach $\alpha=0.83$; anaesthetics, 0.90 ; operation, 0.84 ; unpleasant side effects, 0.7 ; pain, 0.66 ) was used (16). Furthermore, pain intensities at rest and while coughing, and expected pain after the operation were scored, using a $100 \mathrm{~mm}$ visual analogue scale (VAS) anchored to "no pain" and "worst pain I can imagine".

\section{Postoperative pain assessment}

Pain intensity at rest and while coughing was scored one and three hours postoperatively. In case of day-case surgery scoring took place at one hour postoperatively and at the time of discharge. On days $0-4$ after the operation, pain was scored in a pain diary, three times a day. All the inpatients operated upon on the respective day were visited by trained research assistants at 9 P.M. Furthermore research assistants visited all the inpatients at least once a day to give help if necessary.

Day-care patients, and patients from the surgery ward who were discharged from hospital within four days postoperatively, took their pain and medication diary home and returned it to the research team in a special pre-paid envelope. Diaries that had not been returned within 14 days after surgery were followed up by contacting the patient by telephone.

\section{Pain management}

The perioperative pain protocol that has been used at this hospital since 1995 is based on the stepwise approach of acute pain treatment described by Rawal (23). All the operations are categorized into three groups (minor, intermediate and major surgery) based on the anticipated level of postoperative pain. Subsequently, all the surgical procedures were categorized according to the anatomical site. The ENT surgical patients were categorized in the minor and intermediate group. Oral cavity, pharynx, larynx, neck and salivary glands, were categorized in the intermediate group, the anatomical regions ear and nose and endoscopies were mainly categorized as minor operations (Table 1).

In agreement with the prevailing protocol, all patients received paracetamol orally or rectally one hour before induction of anesthesia. After minor operations the patients were treated with paracetamol $(1000 \mathrm{mg})$ four times a day combined with nonsteroidal anti-inflammatory drugs (NSAIDs) administered by the ward nurses. Rescue medicine for moderate or severe pain $(V A S>40)$ was piritramide given intra-
muscularly. 


\section{CHAPTER A}

Table 1. ENT operations divided into anatomical region and endoscopies.

\begin{tabular}{|c|c|c|}
\hline & Anatomical site & Types of operation \\
\hline \multirow[t]{2}{*}{$\begin{array}{l}1 \\
\text { Ear } \\
n=54\end{array}$} & $\begin{array}{l}\text { Outer ear } \\
n=8\end{array}$ & $\begin{array}{l}\text { Incision drainage ear canal, drainaige retroauriculair abcess, } \\
\text { partial excision auricle, } 1^{\text {st }} \text { phase BAHA, mastoid screw, recon- } \\
\text { struction with transtplantation }\end{array}$ \\
\hline & $\begin{array}{l}\text { Middle ear } \\
\mathrm{n}=46\end{array}$ & $\begin{array}{l}\text { Transmeatal drainage and ventilation tubes, inspection- } \\
\text { diagnostic, radical mastoidectomy, sanation middle ear-nos, } \\
\text { stapedectomy, stapedotomy, tympanoplasty type II, tympano- } \\
\text { plasty type I, tympanoplasty type I and ossic chain reconstruc- } \\
\text { tion, tympanoplast facial nerve decompression, cochlear implan- } \\
\text { tation, excision glomus tumor }\end{array}$ \\
\hline \multirow[t]{3}{*}{$\begin{array}{l}2 \\
\text { Nose } \\
n=51\end{array}$} & $\begin{array}{l}\text { Nasal cavity } \\
n=14\end{array}$ & $\begin{array}{l}\text { Extraction nasal polyps, infundib., Extraction nasal polyps, } \\
\text { infundib. 2-sited, polypectomy, conchotomy, conchaluxation } \\
\text { biopsy, conchacauterisaton }\end{array}$ \\
\hline & $\begin{array}{l}\text { Paral nasal sinuses } \\
n=15\end{array}$ & $\begin{array}{l}\text { Endonasal ethmoidectomy, external ethmoidectomy, ethmoid } \\
\text { operation, - nos, sphenoidal exploration, combined operations } \\
\text { moure, Caldwell-luc, endonasal exc tumor - lateral rhinotomy }\end{array}$ \\
\hline & $\begin{array}{l}\text { External nose \& nasal } \\
\text { skeleton } \\
n=22\end{array}$ & $\begin{array}{l}\text { Correction cartilaginous pyramid nos, septal perforation closure, } \\
\text { open rhinoplasty, nasal septum correction-cottle, total resection, } \\
\text { excision tumor/cyst/fistula }\end{array}$ \\
\hline \multirow{4}{*}{$\begin{array}{l}3 \\
\text { Throat } \\
n=22\end{array}$} & Oral cavity & Partial glossectomy, excision malignant tumor mouth floor \\
\hline & $n=4$ & \\
\hline & $\begin{array}{l}\text { Pharynx } \\
n=16\end{array}$ & $\begin{array}{l}\text { Uvulo-pharyngo-palatoplasty, adenotomy, adenoidectomy, } \\
\text { tonsillectomy, laser excision tumor hypopharynx, excision tumor }\end{array}$ \\
\hline & $\begin{array}{l}\text { Larynx } \\
\mathrm{n}=2\end{array}$ & Reduction stenosis or webb, insertion voice prothesis \\
\hline \multirow{2}{*}{$\begin{array}{l}4 \\
\text { Neck } \\
n=28\end{array}$} & $\begin{array}{l}\text { Neck } \\
n=15\end{array}$ & $\begin{array}{l}\text { Radical neckdissection, myotomy, cricopharyngeal muscle } \\
\text { excision, branchial cyst, total laryngectomy }\end{array}$ \\
\hline & $\begin{array}{l}\text { Salivary gland } \\
\mathrm{n}=13\end{array}$ & $\begin{array}{l}\text { Total paroditectomy, lateral paroditectomy, excision other sall- } \\
\text { vary glands, partial paroditectomy, extirpation submandibular } \\
\text { gland }\end{array}$ \\
\hline $\begin{array}{l}5 \\
n=62\end{array}$ & $\begin{array}{l}\text { Endoscopies } \\
n=62\end{array}$ & $\begin{array}{l}\text { Diagnostic pharyngoscopy, esofagoscopy, direct laryngosopy, } \\
\text { microlaryngoscopy, transoral nasopharyngoscopy, therapeutical } \\
\text { microlaryngoscopy, direct laryngoscopy, endoscopic treatment } \\
\text { zenkers diverticukum, esophagus dilatation }\end{array}$ \\
\hline
\end{tabular}

Pain after intermediate operations was treated using the same protocol combined with intravenous piritramide $(2-5 \mathrm{mg})$, which was repeated until the patient reported being pain-free. This was followed by intramuscular piritramide $10-15 \mathrm{mg} 6$ times a day.

All ENT operations were conducted under general anesthesia. 


\section{STATISTICAL ANALYSES}

Actual pain scores (VAS 0 - 100) were used on the day before the operation. Mean pain scores were calculated from each individual, measured on the day of the operation and on postoperative days $0-4$, using the average of the three scores obtained on each of the days. As the pain diaries were sometimes incomplete, totals could vary from day to day. A mean pain score of higher than $40 \mathrm{~mm}$ on a VAS was regarded as being unacceptable (18-20).

To determine which variables independently predict the risk of postoperative pain $\geq 40$, on days 0 to 4 after surgery, we first estimated the association between region of operation and endoscopies with each candidate predictor and the outcome (bivariate analysis). The operation regions and endoscopies, specified in Table 1, were considered very important for prediction of unacceptable pain and therefore remained in the model. All pre-selected candidate predictors with a p-value of $<0.15$ were considered in the multivariable analysis using logistic regression modeling.

Missing values in predictor variables were imputed. This was done because of a presumed non-response problem where the occurrence of missing data is related to the outcome value. Patients, who didn't fill in all data, may have had severe pain at that point in time or in contrast, no pain at all. Either way this could influence the outcome. Missing predictor values were imputed according to the multiple imputation (MI) method described by Rubin et al $(24,25)$. Assuming a Missing At Random mechanism (MAR) each missing value can be imputed using a regression model with the other covariates and outcome as predictor. The imputation is a stochastic process as the value is drawn from a density function generated by the regression model. In this way a complete data set is generated and this process is repeated at least twice. For the present study the imputation procedure was performed 5 times, obtaining 5 complete datasets, with imputed values for expected pain ( $n=4: 1,6 \%)$, surgical fear $(n=6: 2,8 \% \%)$, pain catastrophizing $(n=8: 3,7 \%)$ and optimism $(n=10: 4,6 \%)$ For each of the 5 datasets, that were obtained after Ml, multiple logistic regression analysis was performed to identify the factors that independently predicted the risk of having unacceptable postoperative pain $(\geq 40)$ on the day of surgery and day 1- 4 after operation. Missing values in outcome measures (pain scores) were not imputed. However, these missing data could be subject to selection processes as well. Therefore logistic regression analyses were used to investigate whether these missing outcomes could be predicted with available covariates (ASA grade, preoperative pain, sex etc.). The results revealed some significant relationships and were used to calculate a P-weight factor for each case.

For the multivariable logistic model the continuous quality of the psychological variables was retained. Using a forward entry procedure the criterion for adding a variable was a $p$-value of $>0.05$ based on the log-likelihood ratio test. A variable was included in the final analyses when the variable appeared at least 3 times in the 5 imputation sets. The model's ability to discriminate between patients with and without unacceptable postoperative pain was estimated by the area under the receiver operant curve (AUC). Analyses were performed with STATA (version 8) and SPSS (version 12). 


\section{RESULTS}

Baseline characteristics are presented in Table 2. More male (59\%) patients were included then female $(41 \%)$. Figure 1 indicates the distribution of mean pain scores at the day before surgery until day four postoperatively at rest and while coughing. The ENT operations performed in the region of mouth, throat, neck and salivary glands were painful (VAS $\geq 40$ ) on the day of operation. Approximately $48 \%$ of the patients in these groups have unacceptable mean pain at rest and $58 \%$ have VAS $>40 \mathrm{~mm}$ mean pain while coughing on the day of surgery (Figure 1). For mouth and throat surgery this condition persisted almost throughout the whole studied period.

Of the initial ten predictors other than operation region and endoscopies, only six remained after bivariate analysis i.e. age, gender, preoperative pain, expected pain, surgical fear and pain catastrophizing. The multivariable regression analysis with these six predictors and the anatomical site of the intervention yielded a ROC area of $0.76-0.72$ for the day of surgery until day four (Table 3). Predictors that seemed relevant for the model such as gender, age, and surgical fear were not independent predictors in multivariate analysis. Apparently, the retained predictors already provided for their predictive information. Anatomical site i.e. oral cavity, pharynx, larynx, neck salivary glands and on day 2 also endoscope procedures, preoperative pain on day 1 and pain catastrophizing on day 2-4 remained in the model as predictors of significant higher risk of unacceptable pain. Multivariate analysis indicates that the risk of pain is 4 to 10 times higher in oral cavity, pharynx, larynx, and neck salivary glands surgery groups as compared with the ear surgery category (Table 3 ). Pain catastrophizing is measured on a scale going from 0 to 52 . When comparing two groups, the interpretation of the $O R$ of the pain catastrophizing score is dependent on the difference of this score between these groups; for example an increase of 10 points in the PCS scale would mean an OR or risk increase on POD4 of 1.57 (equals the coefficient 1.046 in table 3 to the power 100). 
PREVALENCE AND PREDICTORS

Table 2. Baseline characteristics

\begin{tabular}{|c|c|c|}
\hline & Number & Perdentage of subjects \\
\hline Total & 217 & \\
\hline \multicolumn{3}{|l|}{ Age $\left(50^{+}\left(16^{\prime}\right)\right.$} \\
\hline$>40$ & 152 & $70 \%$ \\
\hline \multicolumn{3}{|l|}{ Gender } \\
\hline Male & 129 & $59.4 \%$ \\
\hline Female & 88 & $40.6 \%$ \\
\hline \multicolumn{3}{|l|}{ Age 0} \\
\hline $18-39$ & 65 & $30 \%$ \\
\hline $40-59$ & 86 & $39.6 \%$ \\
\hline$>60$ & 66 & $30.4 \%$ \\
\hline Education level & 179 & $83 \%$ \\
\hline Lower & 58 & $26.7 \%$ \\
\hline Middle & 59 & $27.2 \%$ \\
\hline Higher & 62 & $28.6 \%$ \\
\hline Preoperative pain $>40$ & 14 & $6.5 \%$ \\
\hline \multicolumn{3}{|l|}{ Type of admission } \\
\hline Inpatients & 181 & $83 \%$ \\
\hline Outpatients & 36 & $17 \%$ \\
\hline \multicolumn{3}{|l|}{ ASA score } \\
\hline 1 & 105 & $48 \%$ \\
\hline 2 & 80 & $37 \%$ \\
\hline 3 & 30 & $14 \%$ \\
\hline 4 & 2 & $1 \%$ \\
\hline \multicolumn{3}{|l|}{ Surgery time } \\
\hline$<60$ minutes & 126 & $58 \%$ \\
\hline $60-180$ minutes & 77 & $35.5 \%$ \\
\hline$>180$ minutes & 14 & $6.5 \%$ \\
\hline Expected pain $>40$ & 85 & $39 \%$ \\
\hline
\end{tabular}




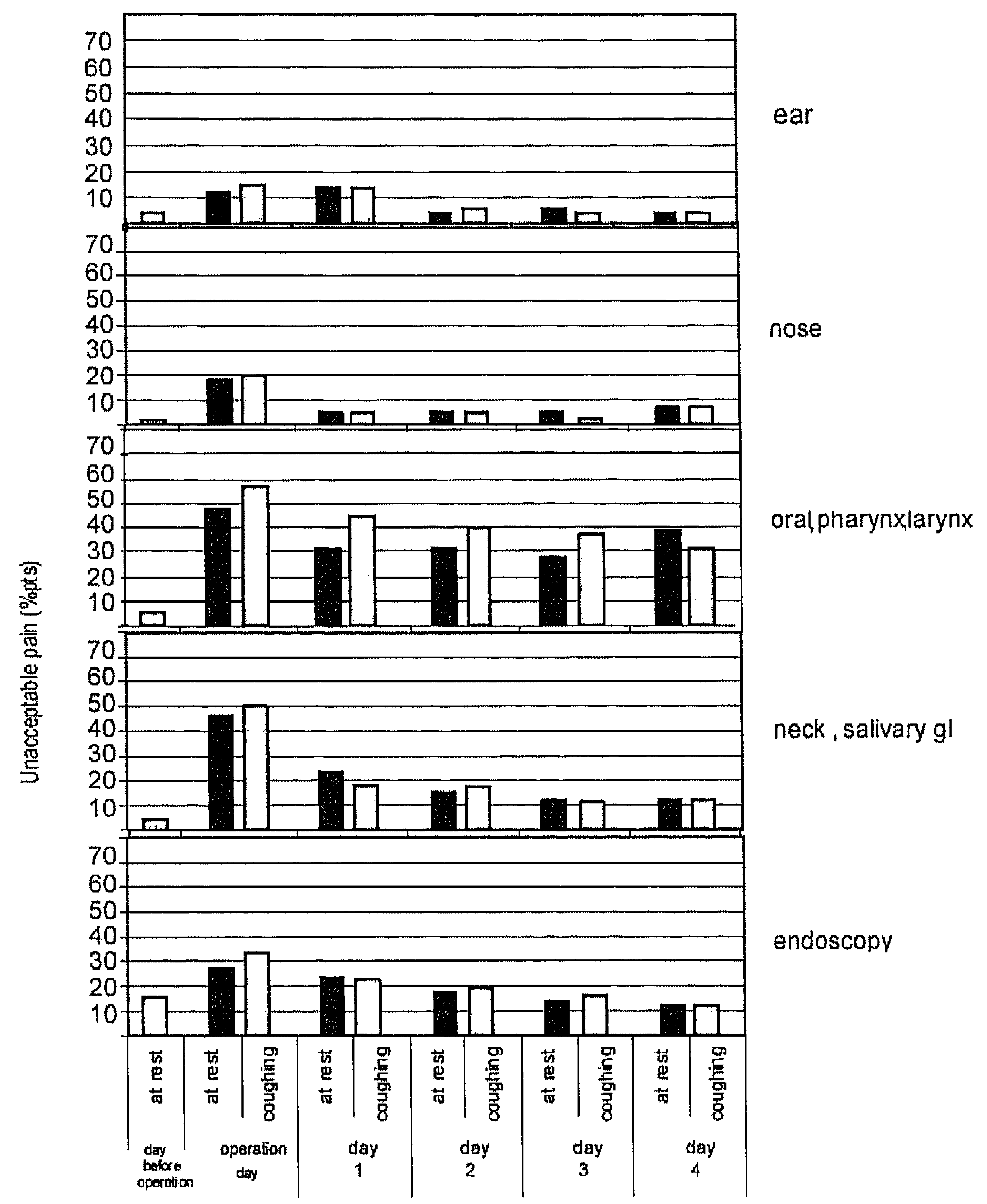

Figure 1. Percentage of patients with pain of $\geq 40 \mathrm{~mm}$ (VAS) at rest on the day before operation and mean pain of $\geq 40 \mathrm{~mm}$ VAS at rest and while coughing on postoperative day $0-4$ after various types of ENT surgery grouped by anatomical site and endoscopies. 
PREVALENCE AND PREDICTORS

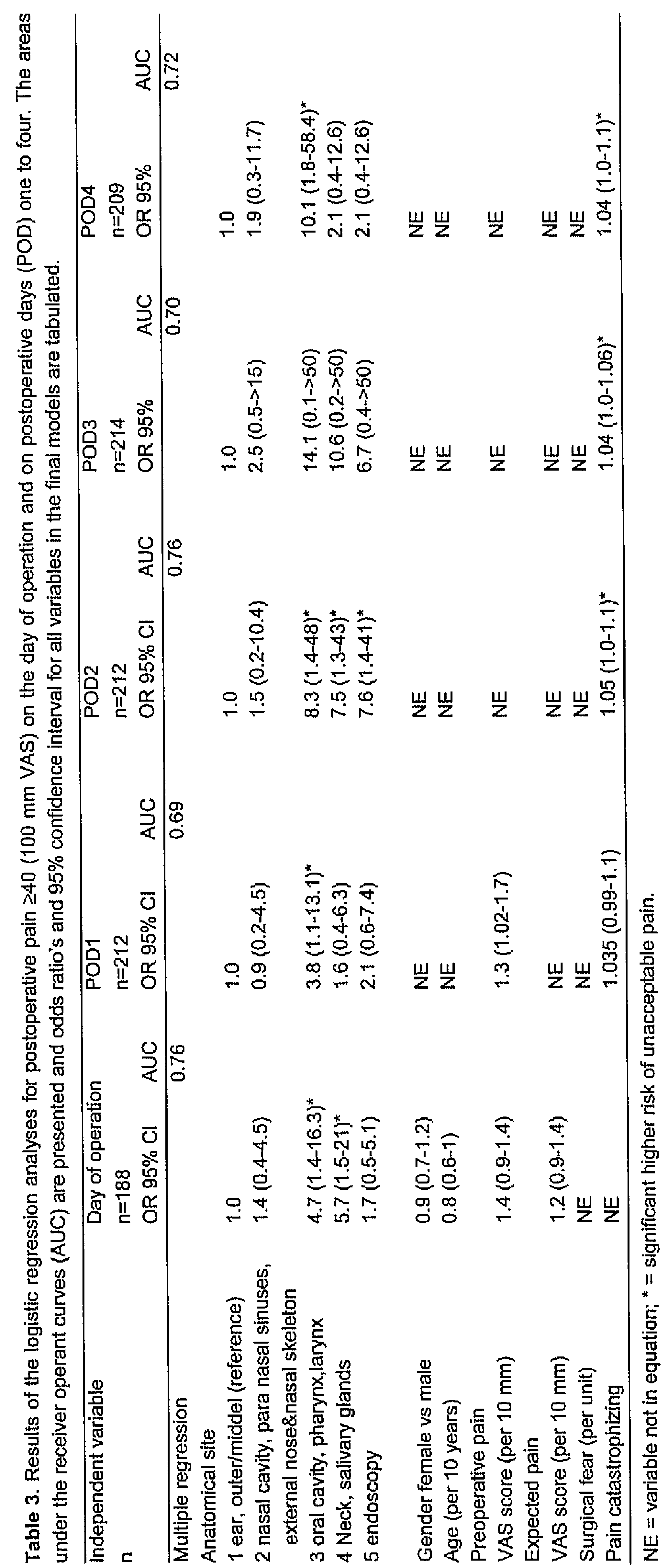




\section{CHAPTER A}

\section{DISCUSSION}

The data from this study demonstrate that there are remarkable differences in postoperative pain sensation at the different anatomical sites of ENT-surgery. A large group of patients has an unacceptable level of pain after surgery on the oral region, pharynx, larynx, neck and salivary glands. Multivariate analysis indicates that the risk of pain is 4 to 10 times higher in these groups as compared with the ear surgery category. The anatomical site seems to play an important role as a predictor of postoperative pain after ENT surgery.

A problem in this study was the presence of missing data on predictor variables $(4 \%)$ and the outcome variable pain (day before surgery $1,6 \%$; postoperative days 1 4 mean 9,6\%). However, for the missing predictors a Multiple Imputation method was used as suggested by Rubin and van Buuren and the missing outcome was adjusted for using $p$ weights as suggested by Rubin $(24,25)$. As the percentage of missing data is low and the imputation method only marginally attributed to the total variance, it was assumed that the missing data hardly affected the results.

Preoperative experimental pain stimuli, such as cold pressure test, electrical skin stimulation or heat stimuli in an attempt to predict postoperative pain were not assessed. Recent data indicate that the response to these stimuli could predict the level of postoperative pain $(12,26-30)$. It would be interesting to measure the predicting power of these tests in relation to the other possible predictors. On the other hand, it is felt that the value of these tests is very limited in a clinical setting. In contrast, use of the PCS and the VAS-scale (to measure preoperative pain) in an attempt to predict postoperative pain is very feasible in this hospital, since every patient has to be screened preoperatively at the pre-assessment policlinic.

In the (oral, pharynx, larynx)-group, pain remains at a high level during the four days of follow-up of this study (OR $3.8-10$ ). A possible explanation, for these high scores, is that 9 out of 22 patients underwent adult tonsillectomy and had pain levels of $>40 \mathrm{~mm}$. This is similar with the finding in the literature, where it was shown that pain after adult tonsillectomy stays high until the seventh day after which it slowly declines $(19,31,32)$.

Conforming to the literature, a low level of pain in the ear-group was found in this study (33).

Multivariate analysis shows preoperative pain as an independent predictor on day of surgery and on POD1. Other studies confirm the value of preoperative pain as an independent predictor $(13,34)$.

In previous studies, expectation of postoperative pain and state anxiety turned out to be independent predictors $(17,27,35)$. In this study, high expectation of pain and state anxiety are not independent predictors. A possible explanation for these conflicting data is that the previous studies did not assess pain catastrophizing. Pain catastrophizing has been defined as an exaggerated negative orientation to aversive stimuli that involves rumination about painful sensations, magnification of the threat value of the pain stimulus, and perceived inability to control pain. It is very likely that, if these studies also had corrected for catastrophizing, expectation of pain and state 
anxiety would no longer have been independent factors or their predictive value would have been substantially reduced. Catastrophizing has been shown to be associated with emotional distress states such as anxiety and depression and expectation of high levels of postoperative pain seems to be the result of catastrophizing $(14,36-$ 39).

Catastrophizing is a good predictor of postoperative pain $(14,28,38)$. Patients with high catastrophizing scores are at risk to suffer from pain longer after surgery. It is well possible that these patients experience pain longer because of their heightened attention to the painful stimulus. The limitations of former studies, investigating the predictive properties of catastrophizing, are the rather small sample sizes $(38,47$ and 48 patients) and the lack of evaluation of the whole range of other determinants that have been imputed predictive values in the literature. In contrast, this study has been built on a relatively large sample size and proves the superiority of catastrophizing in regard to other psychological determinants.

The data of the present study demonstrate that there are large differences in levels of postoperative pain after ENT surgery if the anatomical site is considered. Although some large studies measured the amount of pain in different surgical categories, like abdominal or orthopedic surgery, this study shows that this categorization is probably not sufficient since major differences in pain sensation manifest itself within ENT surgery $(13,40,41)$.

Many endoscopic procedures are considered to be relatively painless and, at worst, associated with mild discomfort. This study seems to prove otherwise. Compared with nose surgery on POD3 there is an OR or risk increase, of unacceptable pain to occur, of 7.6. As yet there is no explanation for this phenomenon.

The presence or absence of unacceptable levels of postoperative pain after general anesthesia in patients undergoing ENT-surgery can be predicted with a limited amount of variables i.e. operation region, preoperative pain and pain catastrophizing. As preoperative use of PCS and the VAS pain scale in an attempt to predict postoperative pain is considered feasible in current clinical practice, given that every patient should be screened preoperatively, these findings can be helpful in improving postoperative pain treatment.

How the catastrophizing postoperative patient should be treated best, with pain medication or otherwise, remains an unanswered question and could be a topic for future research.

The results of this study will assist in improving postoperative pain treatment and in tailoring individual pain management. 


\section{CHAPTER 4}

\section{REFERENCES}

1. Myles PS, Williams DL, Hendrata M, Anderson H., Weeks AM. Patient satisfaction after anaesthesia and surgery: results of a prospective survey of 10,811 patients. Br.J.Anaesth., 2000;84:6-10. .

2. Ballantyne JC, Carr DB, deFerranti S, Suarez T, Lau J, Chalmers TC, Angelillo IF, Mosteller F. The comparative effects of postoperative analgesic therapies on pulmonary outcome: cumulative metaanalyses of randomized, controlled trials. Anesth.Analg. 1998;86:598-612

3. Beattie WS, Buckley DN, Forrest JB. Epidural Morphine Reduces the Risk of Postoperative MyocardialIschemia in Patients with Cardiac Risk-Factors. Canadian Journal of Anaesthesia-Journal Canadien D Anesthesie. 1993;40:532-541.

4. Peters ML, Sommer M, de Rijke JM, Kessels F, Heineman E, Patijn J, Marcus MA, Vlaeyen JW, Van Kleef $M$. Somatic and psychologic predictors of long-term unfavorable outcome after surgical intervention. Ann.Surg. 2007;245:487-494.

5. Marcus MA, Van Kleef $M$, Joosten EA. The quest for new devices to improve postoperative pain control. Anesth.Analg. 2004;99:623-624.

6. Svensson 1, Sjostrom B, Haljamae $H_{1}$ Assessment of pain experiences after elective surgery. J.Pain Symptom Manage. 2000;20:193-201.

7. Raj ed. Management of Postoperative Pain In: Medicine-a comprehensive review. St Louis, MO; Mosby Year Book; Inc. 1993.

8. Husband AD, Davis A. Pain after tonsillectomy. Clin.Otolaryngol.Allied Sci. 1996; 21:99-101.

9. Molony NC, Santana-Hernandez D, Wardrop PJ, Armstrong M, Moralee SJ. On which day is pain worst following adult tonsillectomy. int.J.Clin.Pract. 1998;52:372-373.

10. Chidambaram A, Nigam A, Cardozo AA. Anticipated absence from work ('sick leave') following routine ENT surgery: are we giving the correct advice? A postal questionnaire survey. Clin.Otolaryngol.Allied Sci. 2001;26:104-108.

11. Singh G, McCormack D, Roberts DR. Readmission and overstay after day case nasal surgery. BMC. Ear Nose Throat Disord. 2004;4:2.

12. Bisgaard $T$, Klarskov $B$, Rosenberg J, Kehlet $H$. Characteristics and prediction of early pain after laparoscopic cholecystectomy. Pain. 2001;90:261-269.

13. Kalkman CJ, Visser K, Moen J, Bonsel GJ, Grobbee DE, Moons KG. Preoperative prediction of severe postoperative pain. Pain. 2003;105:415-423.

14. Granot M, Ferber SG. The roles of pain catastrophizing and anxiety in the prediction of postoperative pain intensity: a prospective study. Clin.J.Pain. 2005;21:439-445.

15. Morin C, Lund JP, Villarroel T, Clokie CM. Feine JS. Differences between the sexes in post-surgical pain. Pain. 2000;85:79-85.

16. Edwards RR, Haythornthwaite JA, Sullivan MJ, Fillingim RB, Catastrophizing as a mediator of sex differences in pain: differential effects for daily pain versus laboratory-induced pain. Pain. 2004:111:335-341.

17. Thomas $T$, Robinson $C$, Champion D, McKell M, Pell M. Prediction and assessment of the severity of postoperative pain and of satisfaction with management. Pain. $1998 ; 75: 177-185$.

18. Collins SL, Moore RA, McQuay HJ. The visual analogue pain intensity scale: what is moderate pain in millimetres? Pain. 1997;72:95-97.

19. Dolin SJ, Cashman JN, Bland JM. Effectiveness of acute postoperative pain management: I. Evidence from published data. Br.J.Anaesth. 2002;89:409-423.

20. Jensen MP, Chen $C$, Brugger AM. Interpretation of visual analog scale ratings and change scores: a reanalysis of two clinical trials of postoperative pain. J.Pain. 2003; 4:407-414.

21. Gramke HF, Petry JJ, Durieux ME, Mustaki JP, Vercauteren $M$, Verheecke G, Marcus MA. Sublingual piroxicam for postoperative analgesia: preoperative versus postoperative administration: $a$ randomized, double-blind study. Anesth.Analg. 2006; 102:755-758.

22. Koivula M, Tarkka MT, Tarkka M, Laippala P, Paunonen-llmonen M. Fear and anxiety in patients at different time-points in the coronary artery bypass process. International Journal of Nursing Studies. 2002;39:811-822.

23. Rawal N. Postoperative pain and its management, Ann.Acad.Med.Singapore, 1994;23: 56-64.

24. Rubin DB and Schenker N. Multiple imputation in health-care databases: an overview and some applications. Stat.Med. 1991;10:585-598.

25. van Buuren S, Boshuizen HC, Knook DL. Multiple imputation of missing blood pressure covariates in survival analysis. Stat.Med. 1999;18:681-694. 
26. Hsu YW, Somma J, Hung YC, Tsai PS, Yang CH Chen CC. Predicting postoperative pain by preoperative pressure pain assessment. Anesthesiology. 103;2005:613-618.

27. Pan PH, Coghill R, Houle TT, Seid MH, Lindel WM, Parker RL, Washburn SA, Harris L, Eisenach JC. Multifactorial preoperative predictors for postcesarean section pain and analgesic requirement. Anesthesiology. 2006;104:417-425.

28. Strulov L, Zimmer EZ, Granot M, Tamir A, Jakobi P, Lowenstein L. Pain catastrophizing, response to experimental heat stimuli, and post-cesarean section pain. J.Pain. 2007;8:273-279.

29. Werner MU, Duun $P$ Kehlet $H$. Prediction of postoperative pain by preoperative nociceptive responses to heat stimulation. Anesthesiology. 2004;100:115-119.

30. Wilder-Smith OH, Tassonyi E, Crul BJ, Arendt-Nielsen L. Quantitative sensory testing and human surgery: effects of analgesic management on postoperative neuroplasticity. Anesthesiology. 2003;98:1214-
1222 .

31. Toma AG, Blanshard J, Eynon-Lewis N, Bridger MW. Post-tonsillectomy pain: the first ten days, J Laryngol.Otol. 1995;109:963-964

32. Zagolski OM, Kulisiewicz JE. Pain in patients undergoing day-case ENT surgery. Wlad.Lek. 2005;58:522527.

33. Qureshi AA, Padgham ND, Jiang D. Day-case major ear surgery: is it viable? J.Laryngol.Otol. 2006;120:5-9

34. Gramke HF, de Rijke JM, van Kleef M, Raps F, Kessels AG, Peters ML, Sommer M, Marcus MA, The prevalence of postoperative pain in a cross-sectional group of patients after day-case surgery in a university hospital. Clin.J.Pain. 2007;23:543-548.

35. Mamie C, Bernstein M, Morabia A, Klopfenstein CE, Sloutskis D, Forster A. Are there reliable predictors of postoperative pain? Acta Anaesthesiol.Scand. 2004;48:234-242.

36. Jensen MP, Chen $C$, Brugger AM. Interpretation of visual analog scale ratings and change scores: a reanalysis of two clinical trials of postoperative pain. J.Pain. 2003; 4:407-414.

37. Martin MY, Bradley LA, Alexander RW, Alarcon GS, Triana-Alexander M, Aaron LA, Alberts KR. Coping strategies predict disability in patients with primary fibromyalgia. Pain. 68;1996:45-53.

38. Pavlin DJ, Sullivan MJ, Freund PR, Roesen K. Catastrophizing: a risk factor for postsurgical pain. Clin.J.Pain. 2005;21:83-90.

39. Turner JA, Jensen MP, Romano JM. Do beliefs, coping, and catastrophizing independently predict functioning in patients with chronic pain? Pain. 2000;85:115-125.

40. Miaskowski C, Crews J, Ready LB, Paul SM, Ginsberg B. Anesthesia-based pain services improve the quality of postoperative pain management. Pain. 1999;80:23-29.

41. Sommer M, de Rijke JM, Marcus MA, Kessels F, Peters ML, Geurts JWJM, Gramke HF, Kleef van M. The prevalence of postoperative pain in a sample of 1490 surgical in patients. European Journal of Anaesthesiology. 2008; 25: 267-274. 


\section{Chapter 5}

\section{Somatic and psychological predictors of long-term unfavourable outcome after surgical intervention}

Madelon L. Peters PhD, Micha Sommer MD, Janneke M. de Rijke PhD, Fons Kessels MD MSc, Erik Heineman MD PhD, Jacob Patijn MD PhD, Marco A. E. Marcus MD $P h D$, Johan W.S. Vlaeyen PhD and Maarten van Kleef MD PhD

This study was supported by a grant from the Dutch Foundation of Scientific Research (Zon-MW), grant \# 110000007

Published Annals of Surgery 245, 3:487-494)

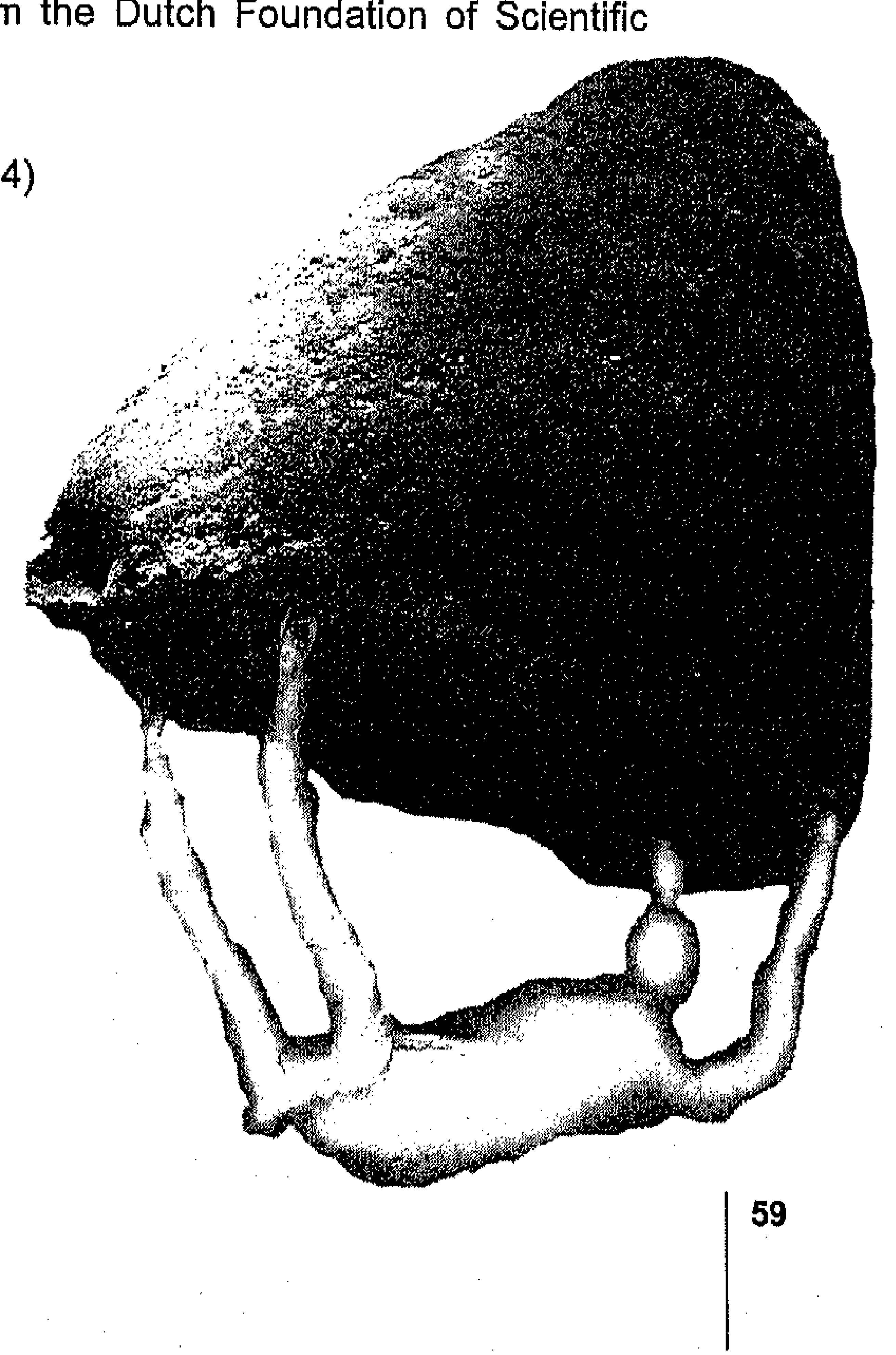




\section{ABSTRACT}

Objective: To identify somatic and psychological predictors of pain, functional limitations, global perceived recovery and quality of life six months after surgical intervention.

Summary Background Data: Recent studies have indicated that chronic pain after surgical intervention is more common than previously assumed. Several demographic and somatic predictors of long-term unfavourable outcome have been identified, but little is known about the contribution of psychological risk factors.

Methods: A prospective cohort study, including 625 patients undergoing elective surgery at the University Hospital Maastricht, The Netherlands, was conducted between February and August 2003. Psychologic questionnaires were completed preoperatively and acute postoperative pain was recorded until 4 days after the operation. Six months later, all patients received follow-up questionnaires to assess pain, functional limitations, global perceived recovery, and quality of life. Multivariable logistic regression analyses were used to estimate relative risk of poor outcome in terms of pain, functional limitations, and global recovery. Multivariable linear regression analysis was used to assess associations with quality of life at 6 months.

Results: The most important somatic predictors of unfavourable outcome were duration of the operation and high levels of acute postoperative pain. Patients reporting high levels of pain 4 days after the operation and patients undergoing an operation of longer than 3 hours were at risk of increased pain, increased functional limitations, poor global recovery, and reported lower levels of quality of life 6 months after the operation. Psychologic variables that influenced long-term outcome were preoperative fear of surgery and optimism. Fear of the long-term consequences of the operation was associated with more pain, poor global recovery, and worse quality of life 6 months later, whereas optimism was associated with better recovery and higher quality of life.

Conclusions: This study was the first to identify the joint contribution of somatic and psychological factors to chronic pain, functional limitations, and quality of life 6 month after surgical interventions. It replicates previous findings that intense acute postoperative pain is a risk factor for long-term adverse outcome and also identified additional risk factors, namely long duration of the operation, ASA status and preoperative fear of surgery. 


\section{INTRODUCTION}

Recent studies have indicated that chronic pain after surgical intervention is more common than previously assumed. Prevalence rates of chronic postoperative pain vary across studies and type of procedures, ranging from approximately $10 \%$ for inguinal hernia operations up to $30-50 \%$ for cardiac surgery ${ }^{1-12}$. A substantial percentage of patients report that this pain interferes with daily activities and leads to physical impairments. ${ }^{1,5,8-10}$

Nerve injury may be one of the causes of chronic postoperative pain, but not all postoperative pain is of neuropathic origin. ${ }^{4,6}$ Another potential cause of sustained pain is neuroplastic change in the central nervou system induced by high intensity pain in the first days following surgery. ${ }^{13}$ Several studies found severe acute postoperative pain to be a risk factor for chronic pain. ${ }^{2,9,11,14}$ Other identified risk factors for sustained postoperative pain are younger age ${ }^{4,6,10}$, female gender ${ }^{15,16}$ and pain before the operation. ${ }^{2,410}$ Psychological factors have also been proposed to influence postoperative pain. Important determinants of acute postoperative pain intensity appear to be preoperative anxiety and catastrophizing (i.e. exaggerated negative beliefs) about pain. ${ }^{17-22}$ However, whether anxiety and catastrophizing also increase the risk of developing chronic postoperative pain remains to be determined. In a review of the literature on predictive factors for chronic postoperative pain, Perkins and Kehlet ${ }^{2}$ conclude that the only reproducible psychological factor contributing to chronicity is neuroticism.

Psychological variables may also affect functional recovery after surgery. Catastrophizing about pain was negatively and optimism and self-efficacy were positively associated with the speed of functional recovery after various types of surgical interventions. ${ }^{23-27}$ Long-term effects of psychological variables on physical disability and well-being have also been reported. ${ }^{16,25,28,29}$

For the present study, preoperative somatic and psychological variables and acute postoperative pain were tested as predictors of pain, functional limitations, global perceived recovery, and health-related quality of life at 6 months follow-up $(\mathrm{FU})$ in patients undergoing various surgical interventions. By combining somatic and psychological predictors in a single study the relative contribution of a certain variable after controlling for other variables can be established. Moreover, multiple long-term outcome variables are included allowing assessment of the possible differential predictive power of somatic and psychological factors for different outcomes.

\section{METHODS}

\section{Patients}

The study was approved by the Ethical Committee of the University Hospital Maastricht, The Netherlands. From February to August 2003, 1975 patients scheduled for elective surgery at the University Hospital Maastricht were approached for participa- 


\section{CHAPTER 5}

tion. Inclusion criteria were: age $>18$, understanding of Dutch, surgery at the departments of general surgery, plastic surgery, orthopaedics, ophthalmology, gynaecology, ear-nose-throat, maxillo-facial surgery, urology, neurosurgery, or thoracic surgery. Exclusion criteria were: limitations of self-expression, visual dysfunction, emergency surgery, cardiac surgery or caesarean section. Informed consent for preand acute postoperative assessment was obtained from 1663 patients (85\%). Patients $(n=173)$ were excluded from further participation for the following reasons: complications during surgery $(n=14)$, the need for extended postoperative ventilatory support at the intensive care unit $(n=50)$, research too demanding $(n=32)$, missing data $(n=33)$, or logistic reasons (e.g. no research assistant available; $n=44$ ). Of the 1490 remaining patients, 1003 gave informed consent to participate in the follow-up study. Follow-up questionnaires were completed by 838 patients. A further 213 patients were excluded because of events occurring in the follow-up period that had a potentially detrimental effect on health but were unrelated to surgical intervention (e.g. a car accident; $n=89$ ) or because they had repeated operations $(n=124)$. This left 625 patients for follow-up analyses. Mean age of patients was 54.8 year and $53 \%$ was female.

\section{Preoperative assessment}

Eligible patients received a letter describing the purpose and methods of the study and a set of questionnaires 1 to 3 weeks preceding the operation. The following questionnaires were included: (1) Pain catastrophizing scale (PCS): 13 items measuring exaggerated negative interpretation of the meaning of pain; ${ }^{30}$ (2) Behavioural inhibition scale (BIS): 7 items measuring anxious temperament; ${ }^{31}$ (3) Life Orientation Test (LOT): 8 items measuring the personality trait optimism; ${ }^{32}$ (4) General SelfEfficacy Scale (GSES), which measures the generalized belief that one has the skills to reach personal goals (10 items); ${ }^{33}$ and (5) Medical Outcomes Study Short-Form General Health Survey (SF-36), measuring 8 domains of health-related quality of life (pain interference, physical functioning, social functioning, role limitations functional, role limitations social, vitality, mental health, and general health $)^{34}$. Completion of questionnaires took 15-20 minutes. Patients were requested to bring the completed questionnaires to the hospital on the day of admission.

One day before the operation patients were visited on the ward by a research assistant who obtained informed consent and scored patients' preoperative pain on a $100 \mathrm{~mm}$ visual analogue scale (VAS). Patients also filled out one additional questionnaire on surgical anxiety. This 10 -item questionnaire was adapted from Koivula et al. $^{35}$ who studied fear of coronary bypass surgery. For the present study, cardiac specific items and one item referring to death were removed.

\section{Postoperative assessment}

Operations were independently catagorized by 5 experienced anesthetists into three groups (minor, intermediate and major) based on the anticipated level of postopera- 
tive pain and in accordance with Rawal's stepwise approach of acute pain treatment ${ }^{36}$ (table 1). Subsequently, surgical procedures were categorized according to the anatomic region of the body involved (head/neck, upper extremities, thoraxnoncardiac, abdominal high, abdominal low, abdominal high and low, lower extremities, back, multiple sites). Type of anaesthesia was coded as general, locoregional or general plus locoregional. Duration of the operation was dichotomized as $<3$ or $\geq 3$ hours. In addition, acute postoperative pain during the first 4 days after the operation was assessed. Pain intensity at rest, during deep breathing and with coughing was scored 3 times a day on a $100 \mathrm{~mm}$ VAS.

Six months after surgery participants were sent the long-term recovery questionnaire and the SF-36 by mail. We devised the long-term recovery questionnaire for the purpose of this study. It included the 1-item Global Surgical Recovery (GSR) index ("If $100 \%$ recovery is back to the usual health you had before you got sick and had surgery, what percent of recovery you are at now?"). The GSR has been used previously and was found to correlate 0.72 with a more elaborate instrument of surgical recovery ${ }^{37}$. Next were 4 questions to measure changes due to the operation in pain, mobility (walking, sitting and lying down), self-care (washing and dressing) and activity level (sports and physical exertion). Each of these questions started by asking whether the patient experienced difficulties in the specified domain before the operation and whether he/she experienced difficulties at FU. When either of these questions was answered affirmatively the respondent was asked how the operation influenced the problem (much less, somewhat less, the same, somewhat more, much more). Presence of preoperative pain (no/yes) was also established by this questionnaire.

\section{Statistical analyses}

The 4 outcome variables in the study were increased pain due to the operation (no/yes), increased functional limitations due to the operation (no/yes), poor global recovery (no/yes) and quality of life (continuous). Blockwise logistic regression analysis was used to identify the factors that were associated with the dichotomous outcome variables. In the first step 2 variables describing the surgical procedure were entered: anticipated postoperative pain level and body region were the operation took place. In step 2 the demographic variables age, sex, and education were entered in the model. The contribution of the somatic variables preoperative pain, duration of the operation, anesthetic technique, acute postoperative pain, and ASA grade was examined in step 3 . The psychologic variables were entered to the model in the final step. Psychologic variables were dichotomized by median split. Variables in step 1 and 2 were entered using a forced entrance procedure and variables in step 3 and 4 were entered with a forward procedure (likelihood ratio), using a criterium of $p<0.10$ for entering variables in the model. The significance of each step was tested using the $x^{2}$ statistic. The models' ability to discriminate between patients with a favourable and a nonfavourable outcome was estimated by the area under the receiver operant curve (AUC) for the succesive steps. 


\section{CHAPTER 5}

Table 1. Operations subdivided in anticipated postoperative pain level and anatomical region

\begin{tabular}{|c|c|c|}
\hline $\begin{array}{l}\text { Anticipated post- } \\
\text { operative pain } \\
\text { level }\end{array}$ & Anatomical region & Type of operation \\
\hline \multirow[t]{6}{*}{ Minor } & Head and neck & $\begin{array}{l}\text { Thyroidectomy, stapedectomy, tympanoplasty, petrosal } \\
\text { bone and middle ear surgery, cochleair implantation, } \\
\text { middle ear inspection, middle ear reconstruction, auricle } \\
\text { operation, nasal sinus operation, endoscopy of pharynx, } \\
\text { larynx, bronchus, craniotomy, eye surgery }\end{array}$ \\
\hline & Upper extremities & Peripheral vascular operations, woundtollet, sutures \\
\hline & Thorax- noncardiac & Vascular operations, woundtoilet \\
\hline & Lower abdomen/pelvis & $\begin{array}{l}\text { Vaginal urological procedures, cervix operation, abortion, } \\
\text { hysteroscopy, operations of male genital, endoscopic } \\
\text { urological interventions like TUR, cystic biopsy, urethra, } \\
\text { cystoscopy, plastic skin operations }\end{array}$ \\
\hline & Lower extremities & $\begin{array}{l}\text { Plastic skin operations, peripheral vascular operations, } \\
\text { woundtoilet }\end{array}$ \\
\hline & Back/Spinal & Urological neuromodulation, small plastic skin operations \\
\hline \multirow[t]{8}{*}{ intermediate } & Head and neck & $\begin{array}{l}\text { Neck dissection, mouth and throat surgery, laryngec- } \\
\text { tomy, pharynx and larynx surgery, maxilla and mandibu- } \\
\text { lar surgery }\end{array}$ \\
\hline & Upper extremities & Orthopaedic hand and arm surgery, \\
\hline & Thorax- noncardiac & Plastic breast surgery \\
\hline & Upper abdomen & $\begin{array}{l}\text { Fundoplication, duodenum surgery, cholecystectomy, } \\
\text { nephrectomy }\end{array}$ \\
\hline & lower abdomen/pelvis & $\begin{array}{l}\text { Surgery of vulva, ovary, adnexen, vaginal and abdomi- } \\
\text { nal uterusextirpation, abdominal endometriosis, closure } \\
\text { of anus praeter, colorectal surgery, anal surgery, ab- } \\
\text { dominal vascular surgery, abd. lipectomy, cystic resec- } \\
\text { tion, Ryntshak and radical prostatectomy }\end{array}$ \\
\hline & Lower extremities & $\begin{array}{l}\text { Plastic skin operations, orthopaedic surgery } \\
\text { foot/knee/leg/hip, amputation, peripheral vascular } \\
\text { surgery }\end{array}$ \\
\hline & Back/spinal & $\begin{array}{l}\text { Plastic skin operations, spinal cord decompression with } \\
\text { hemilaminectomy, discectomy }\end{array}$ \\
\hline & $>1$ region & $\begin{array}{l}\text { Plastic breast + abdominal surgery, arm/hand and hip } \\
\text { (fractures) }\end{array}$ \\
\hline \multirow[t]{5}{*}{ Major } & Upper extremities & Shoulder surgery (endoprothesis), clavicle surgery \\
\hline & Thorax- noncardiac & Lobectomy \\
\hline & Upper+ Lower abdomen & $\begin{array}{l}\text { Hepatectomy, pancreatectomy, combination of different } \\
\text { bowel surgery, aortic surgery, extensive gynaecological } \\
\text { surgery, combination of bowel and gynaecological } \\
\text { surgery }\end{array}$ \\
\hline & Lower extremities & Total knee replacement \\
\hline & Back/spinal & Spondylodeses, untethering \\
\hline
\end{tabular}


Blockwise linear regression analysis was used to analyze the continuous variable quality of life (QOL). In step 1 the variables describing the surgical procedure were entered, together with preoperative level of $\mathrm{QOL}$, to control for baseline differences. Step 2 entered the demographic variables, step 3 the somatic variables and step 4 the psychological variables. For the linear regression analysis, the continuous quality of the psychological variables was retained. Step 3 and 4 again used a forward entry procedure with $p<0.10$ as the criterium for adding a variable. The significance of each step was tested using $F$ change. The total amount of explained variance in QOL at follow-up is indicated by $\mathrm{R}^{2}$. Analyses were performed with the Statistical Package for Social Sciences (SPSS), version 11.5.

\section{Ethical considerations}

Before the operation patients were asked for consent to participate in a study including preoperative assessment and data collection of relevant variables until 4 days postoperatively. Separate informed consent was obtained for the follow-up part of the study 4 days after the operation.

\section{RESULTS}

\section{Dropout analyses}

Compared to the initial sample of 1490 patients, study participants had somewhat higher education $(29.7 \%$ low education, $40.4 \%$ intermediate and $29.9 \%$ high; in the initial sample these numbers were: $33.2,37.9$ and 28.9). Moreover, they less often had a major operation ( $17.8 \%$ vs. $20 \%$ ), remained fewer days in hospital (6.0 days versus 6.8 days) and were in better physical health before the operation (ASA grade l: $41.9 \%$ versus $36.1 \%$ in initial sample).

\section{Data reduction and validity of predictor variables}

The surgical anxiety questionnaire was subjected to principal component analysis with oblimin rotation to identify its factor structure. Two components were found that explained $60 \%$ of total variance (eigenvalues 4.8 and 1.2 ). The first component consisted of six items concerning the following fears (item loadings in parenthesis): incomplete recovery $(0.93)$, long duration of rehabilitation $(0.83)$, non-succesful operation (0.78), adverse health effect $(0.77)$, worries about family members $(0.47)$ and stay in hospital (0.43). Together these items constituted a subscale that was termed "fear of long-term consequences of the operation" (Cronbach $\alpha=0.82$ ). The second component contained 4 items with fears concerning: anaesthesia $(0.90)$, the operation itself (0.84), unpleasant side effects $(0.70)$ and pain (0.66). This subscale was termed "fear of immediate consequences of the operation" (Cronbach $\alpha=0.83$ ). The intercorrelation between the subscales was 0.56 . 


\section{CHAtras 5}

To check the validity of our retrospective measure of preoperative pain, we compared the preoperative pain intensity VAS and the SF-36 pain score of patients who did or did not report preoperative pain on the long-term follow-up questionnaire. The frequency distribution of the preoperative VAS and SF-36 pain scores showed clearly different patterns for the two groups in line with expectations. Patients reporting no preoperative pain retrospectively had an average preoperative pain score of 4 on the VAS and 84 on the SF-36 pain subscale, while patients with preoperative pain scored 22 on the VAS and 45 on the SF-36 pain subscale.

To quantify acute postoperative pain we calculated an average pain intensity score for each of the four postoperative days on the basis of the VAS pain at rest. The three different pain scores (rest, coughing, breathing) were highly correlated (average Pearson $\mathrm{R}=.87$, range $0.75-0.98$ for the various time points) and only pain at rest had no missing values. For the logistic regression analyses average pain intensity was transformed into a dichotomous variable according to VAS $<40$ or $\geq 40$.

\section{Increased pain}

The outcome variable "increased pain due to the operation" was derived from the long-term recovery questionnaire and operationalized as "somewhat" or "much more" pain at FU than before the operation. More than twelve percent of patients $(n=78$; $12.5 \%$ ) reported increased pain 6 months after the operation, i.e. their preoperative pain had become worse $(n=37)$ or they had developed a new pain problem $(n=41)$.

Blockwise multiple logistic regression analysis was used to test for significant predictors of increased pain. The step 1 variables anticipated postoperative pain level and body region yielded an AUC of $0.66\left(X^{2}=23.8, p=0.008\right)$. The inclusion of demographic variables in step 2 did not significantly improve the model $\left(X^{2}=1.2, p=0.88\right.$, $A \cup C=0.67)$. The somatic variables entered in the third step did significantly contribute to the model $\left(X^{2}=29.4, p=0.001, A \cup C=0.74\right)$. In the final step fear of long-term consequences of the operation entered the model, increasing discriminative ability of the model to $A \cup C=0.75\left(X^{2}=5.1, p=0.024\right)$. Table 2 shows the results for the variables in the last two steps. In the final model, acute postoperative pain (4 days after the operation, $\mathrm{OR}, 3.21 ; 95 \% \mathrm{Cl}, 1.6-6.3)$ and preoperative pain (OR, $0.32 ; 95 \% \mathrm{Cl}, 0.17$ $0.60)$ were most strongly associated with increased pain at $\mathrm{FU}$, followed by anticipated postoperative pain level (OR, 2.6;95\% Cl, 1.2-5.8 for intermediate vs minor procedures) and duration of the operation (OR, 2.0;95\% Cl, 1.0-4.0) ${ }^{\#}$. High acute post operative pain, intermediate surgical procedures and longer operations increased

\footnotetext{
\& Validity of this measure was demonstrated by comparing the mean change in the SF-36 pain subscale from pre-operation to follow-up. Patients reporting more pain at FU indeed had decreased scores on the SF-36 pain subscale ( 43 at FU vs. 56 preoperatively), patients reporting no change in pain obtained equal SF-36 pain scores ( 81 vs. 80 ) and patients reporting less pain showed improvement on the SF-36 pain subscale (75 at follow-up vs. 48 preoperatively).

\# Acute postoperative pain scores on day 1 to 4 were used as independent variables in successive analyses. Each of these variables was significantly related to increased pain at FU, but pain on day 4 most strongly $(\mathrm{OR}=2.4$ for day $1,2.8$ for day $2,2.5$ for day 3 and 3.2 for day 4 ). In all further analyses, pain intensity on day
} 
Table 2. The association of step 3 and 4 predictor variables with outcome variables increased pain at 6 months FU, increased functional limitations at 6 months FU and poor global recovery. Odds ratios (ORs) and the $95 \%$ confidence interval for the variables in the final model are given.

\begin{tabular}{|c|c|c|c|c|c|c|c|}
\hline \multirow[t]{2}{*}{ Independent variable } & \multirow[b]{2}{*}{$N$} & \multicolumn{2}{|c|}{ Increased Pain at FU } & \multicolumn{2}{|c|}{$\begin{array}{l}\text { Increased Functional } \\
\text { Limitations at FU }\end{array}$} & \multicolumn{2}{|c|}{ Poor Global Recovery } \\
\hline & & OR $(95 \% \mathrm{Cl})$ & $P$ & OR $(95 \% \mathrm{Cl})$ & $\mathrm{P}$ & OR $(95 \% \mathrm{Cl})$ & $\mathrm{P}$ \\
\hline \multicolumn{8}{|l|}{ Preoperative pain } \\
\hline No & 274 & 1.00 & & 1.00 & & 1.00 & \\
\hline Yes & 351 & $0.32(0.17-0.60)$ & 0.001 & NE & & $1.90(1.21-2.97)$ & 0.01 \\
\hline \multicolumn{8}{|l|}{ Duration of Surgery } \\
\hline$<3$ hours & 545 & 1.00 & & 1.00 & & 1.00 & \\
\hline$\geq 3$ hours & 80 & $2.00(1.01-3.97)$ & 0.05 & $4.24(2.33-7.71)$ & 0.001 & $2.45(1.37-4.39)$ & 0.01 \\
\hline \multicolumn{8}{|l|}{ Pain on day 4} \\
\hline Pain $<40$ & 551 & 1.00 & & 1.00 & & 1.00 & \\
\hline Pain $\geq 40$ & 74 & $3.21(1.64-6.30)$ & 0.001 & $1.87(1.02-3.41)$ & 0.04 & $2.61(1.47-4.62)$ & 0.001 \\
\hline \multicolumn{8}{|l|}{ ASA status } \\
\hline ASA grade I & 262 & 1.00 & & 1.00 & & 1.00 & \\
\hline ASA grade II & 274 & NE & & $1.38(0.80-2.38)$ & 0.24 & NE & \\
\hline ASA grade III & 89 & NE & & $2.40(1.17-4.90)$ & 0.02 & NE & \\
\hline \multicolumn{8}{|l|}{ Long-term Fear } \\
\hline Low $(<10)$ & 304 & 1.00 & & 1.00 & & 1.00 & \\
\hline High $(\geq 10)$ & 321 & $1.90(1.08-3.33)$ & 0.03 & NE & & $1.98(1.34-2.94)$ & 0.001 \\
\hline \multicolumn{8}{|l|}{ Optimism } \\
\hline Low $(<28)$ & 269 & 1.00 & & 1.00 & & 1.00 & \\
\hline High $(\geq 28)$ & 283 & $\mathrm{NE}$ & & NE & & $0.60(0.41-0.87)$ & 0.01 \\
\hline
\end{tabular}

$\mathrm{NE}=$ variable not entered in the equation

the risk of increased pain at FU, while pain before the operation decreased the risk. Fear of the long-term consequences of surgery further elevated the risk of increased pain at $\mathrm{FU}(\mathrm{OR}, 1.9 ; 95 \% \mathrm{Cl}, 1.1-3.3)$.

\section{Increased functional limitations}

Increased functional limitations due to the operation was scored from the longterm recovery questionnaire. Seventeen percent of patients $(n=105)$ reported that due to the operation they had "somewhat" or "much more" problems with mobility, self-care or physical activity at FU than before the operation. $\phi$

\footnotetext{
Validity of this retrospective report was checked by comparing the preoperative and follow-up SF-36 physical functioning score of patients with more, same and less perceived functional limitations. As expected, patients with more limitations at FU scored lower on the SF-36 physical functioning scale ( 49 at FU vs. 55 preoperatively), patients with same limitations had an identical score (79 at both assessments) and patients with less limitations scored higher on the SF-36 physical functioning scale (66 at FU vs, 49 preoperatively).
} 


\section{CHAPTER 5}

Blockwise logistic regression analyses indicated that the surgical procedure variables entered in step 1 yielded a sigificant predictive model with an AUC of $0.68\left(X^{2}=39.9\right.$ $p=0.001)$. The step 2 demographic variables did not improve the model $\left(X^{2}=2.94\right.$ $p=0.57, A \cup C=0.69)$ but the step 3 somatic variables did $\left(X^{2}=32.91, p=0.001\right.$ $A \cup C=0.75$ ). None of the psychological variables entered the model in step 4 . The variables associated with increased functional limitations at FU were anticipated postoperative pain level $(\mathrm{OR}, 2.7 ; 95 \% \mathrm{Cl}, 1.3-5.8$ for intermediate vs minor procedures; OR, $3.3 ; 95 \% \mathrm{Cl}, 1.2-9.2$ for major vs minor procedures), ASA status (OR, $2.4 ; 95 \% \mathrm{Cl}, 1.2-4.9$ for ASA grade III vs grade I), duration of the operation (OR, 4.2; $95 \% \mathrm{Cl}, 2.3-7.7$ ), and pain 4 days after the operation (OR, $1.9 ; 95 \% \mathrm{Cl}, 1.0-3.4$ ). Results for step 3 and 4 predictor variables can be found in table 2 .

\section{Poor global recovery}

On average, patients reported to have recovered $78 \%$ (SD: 25 ; range: $0-100$ ) on the global surgical recovery index. Poor global recovery was defined as a score of $80 \%$ or less on this index. According to this criterion, 215 patients $(34.4 \%)$ reported poor recovery at 6 months post-operation.

The logistic regression analysis demonstrated that poor global recovery was significantly predicted by the variables coding the surgical procedure $\left(X^{2}=63.5\right.$ $p=0.001, A \cup C=0.68)$. Demographic variables improved the model $\left(X^{2}=12.8, p=0.01\right.$ $A \cup C=0.71)$. The somatic variables in step 3 increased the $A \cup C$ to $0.76\left(X^{2}=41.9\right.$ $p=0.001)$ and the psychological variables in step 4 to $0.77\left(X^{2}=20.0, p=0.001\right)$. The variables coding surgical procedure and the somatic variables in the full regression model associated with poor recovery were body region (upper extremities/shoulder operations associated with poor outcome, OR, 2.9; 95\% Cl, 1.2-6.8; lower abdominal operations associated with good outcome, OR, 0.42;95\% Cl, 0.25-0.73), duration of the operation (OR, 2.4;95\% Cl, 1.4-4.4), pain 4 days after the operation (OR, 2.6 $95 \% \mathrm{Cl}, 1.5-4.6)$ and preoperative pain (OR, $1.9 ; 95 \% \mathrm{Cl}, 1.2-3.0)$. Female sex was associated with a somewhat better global recovery (OR, $0.67 ; 95 \% \mathrm{Cl}, 0.44-0.98$ ). Psychological variables further contributed to the model with long-term fear of the consequences of surgery being associated with poor global recovery $(\mathrm{OR}, 2.0 ; 95 \%$ $\mathrm{Cl}, 1.3-2.9$ ) and optimism with good recovery (OR, 0.60;95\% Cl, 0.41-0.87). See table 2 for an overview of the step 3 and 4 variables.

\section{Quality of life}

In order to reduce the number of outcome variables related to quality of life, principal component analysis was conducted on the eight subscales of the SF-36. All subscales loaded on a single factor (eigenvalue 5.0 ), explaining $62.4 \%$ of variance (individual factor loadings ranging between 0.73 and 0.88 ). The aggregated $\mathrm{QOL}$ score had high internal consistency (Cronbach $\alpha=0.89$ ) and was used in further analyses. 
PREOHCTOS OF LOMG

Table 3. Results of the linear regression analysis for the aggregated SF-36 quality of life score at 6-months follow-up. Significance and $\mathrm{R}^{2}$ for each of the steps in the model is shown together with the standardized betas for all variables in the final model.

\begin{tabular}{|c|c|c|c|}
\hline & $\mathrm{R}^{2}$ & F change & $P$ \\
\hline Step1 & 0.368 & 31.7 & 0.001 \\
\hline Step 2 & 0.384 & 3.81 & 0.05 \\
\hline Step 3 & 0.434 & 13.1 & 0.001 \\
\hline \multirow[t]{2}{*}{ Step 4} & 0.448 & 7.31 & 0.001 \\
\hline & Stand. B & $P$ & \\
\hline Preoperative QOL & 0.456 & 0.001 & \\
\hline $\begin{array}{l}\text { Anticipated postop pain } \\
\text { level }^{1}\end{array}$ & $\cdot$ & & \\
\hline Intermediate & 0.063 & 0.15 & \\
\hline Major & 0.050 & 0.36 & \\
\hline \multicolumn{4}{|l|}{ Body region ${ }^{2}$} \\
\hline Head/neck & 0.243 & 0.09 & \\
\hline Upper extremities & 0.090 & 0.20 & \\
\hline Thorax-noncardiac & 0.067 & 0.32 & \\
\hline Abdominal High & 0.082 & 0.22 & \\
\hline Abdominal Low & 0.283 & 0.04 & \\
\hline Abdominal $\mathrm{H}+\mathrm{L}$ & 0.180 & 0.07 & \\
\hline Lower extremities & 0.229 & 0.08 & \\
\hline Back & 0.134 & 0.17 & \\
\hline Age & -0.014 & 0.69 & \\
\hline Sex & -0.005 & 0.88 & \\
\hline \multicolumn{4}{|l|}{ Education $^{3}$} \\
\hline Low & -0.092 & 0.02 & \\
\hline Middle & -0.077 & 0.04 & \\
\hline Duration of Surgery & -0.094 & 0.01 & \\
\hline Pain intensity day 4 & -0.169 & 0.001 & \\
\hline \multicolumn{4}{|l|}{ ASA status $^{4}$} \\
\hline ASA grade II & -0.062 & 0.09 & \\
\hline ASA grade III & -0.127 & 0.001 & \\
\hline Long-term fear & -0.080 & 0.02 & \\
\hline Optimism & 0.090 & 0.01 & \\
\hline
\end{tabular}

${ }^{1}$ Reference category: minor

${ }^{2}$ Reference category : multiple sites

${ }^{3}$ Reference category: high education

${ }^{4}$ Reference category: ASA grade I 


\section{CHAPTER:}

On average, patients showed improved quality of life at 6 months $F U$, with an aggregated QOL score of 62 (sd: 22.2) pre-operation and 67.9 (SD: 21.7) at FU. Linear regression analysis indicated that most of the variance in QOL at FU (37\%) was explained by the variables in step 1 and especially preoperative QOL. The demographic variables in step 2 significantly contribute to the model,explaining an additional $2 \%$ variance, while the somatic variables in step 3 explained $5 \%$ of unique variance. Lower education, higher pain intensity at day 4 postoperatively, longer duration of the operation and ASA grade III were associated with decreased QOL, and lower abdominal operations were associated with increased QOL at FU. Step 4 added $1 \%$ of unique explained variance. Higher fear of the long-term consequences of the operation was assocatied with lower QOL and higher optimism with higher QOL. The final model could explain $45 \%$ of the variance in QOL at FU. Results are shown in table 3.

\section{DISCUSSION}

A total of 625 patients undergoing various types of surgical intervention were followed for 6 months to establish predictors of adverse outcome. Several somatic and psychological predictors of increased pain, functional limitations, global recovery and quality of life at FU were identified by means of blockwise regression analysis in which clusters of variables were added to the prediction model.

The first cluster consisted of 2 variables coding the surgical procedure: anticipated (acute) postoperative pain level and body region of the operation. Operations classified as "intermediate" in terms of anticipated painfulness were significantly more often associated with increased pain and increased functional limitations at 6 months FU compared to minor operations. "Major" operations were associated with increased functional limitations but not with increased pain at FU. It should be noted that according to the prevailing protocol of postoperative pain management the classification into minor, intermediate and major has implications for acute postoperative pain treatment. Procedures with higher anticipated postoperative pain levels were followed by stronger analgesic interventions, i.e. procedures classified as major were treated with continuous epidural infusion of bupivacaine $0.125 \%$ with sufentanil $1 \mu \mathrm{g} / \mathrm{ml}$ or with patient controlled intravenous administration of piritramide. Therefore actual postoperative pain level after major operations was in many instances not higher than after procedures classified as minor or intermediate.

Anatomic region of the body involved was not a major predictor of outcome. Neither increased pain nor increased functional recovery were associated with operations in a specific body region. Global recovery and quality of life at FU did however show an association with body region: patients with upper extremity/shoulder operations had an elevated risk of poor global recovery and patients with lower abdominal operations had a lower risk of poor global recovery and reported increased levels of QOL at FU. It should be admitted that our classification into body regions was fairly 
crude, clustering sometimes heterogeneous procedures into the same category, but group sizes did not allow further differentiation.

The contribution of demographic variables was tested in the second step of the regression models. Younger age and female gender have been associated with an increased risk of chronic postoperative pain in previous studies but these variables appeared unrelated to most of the outcome variables in the present study. Female gender even appeared to be protective against poor global recovery.

Various of the somatic variables that were added to the model in the third step showed a relatively strong assocation with outcome. Operations with a duration longer than 3 hours had a significantly higher chance of increased pain, increased functional limitations and poor global recovery 6 months after surgery. Duration of the operation also influenced the change in quality of life from pre-operation to 6 months FU. Another prominent predictor of long-term unfavourable outcome was a high level of acute postoperative pain. Patients with a VAS $\geq 40$ on postoperative day 4 had a higher chance of experiencing increased pain, increased functional limitations and poor global recovery 6 months after the operation. High acute postoperative pain also negatively affected quality of life 6 months after surgery. These results coincide with previous findings demonstrating that more intense acute postoperative pain may be a risk factor for the development of chronic pain ${ }^{2,3,9,11,14}$ and delays functional recovery 6 months later. ${ }^{7}$ The strong assocations found between both the duration of the operation and acute postoperative pain and unfavourable outcome may point to the role of central sensitization. Longer operations are associated with more enduring nociceptive barrage during surgery while high acute postoperative pain levels lead to intense and enduring nociceptive input in the postoperative period. Both may increase the chance of central sensitization, and subsequently persistent pain. ${ }^{13,38}$

An inconsistent finding arose for the influence of preoperative pain. Having pain before the operation was associated with a lower chance of increased pain at FU, but it increased the chance of poor global recovery. Previous studies have found preoperative pain to increase the chance of chronic pain. ${ }^{2,3,10}$ ASA status was significantly associated with increased functional limitations and the change in QOL 6 months after the operation. Patients with ASA grade III experienced more functional limitations and showed less improvement in QOL at FU compared to patients with ASA grade $\mathrm{I}$. Thus, being in a poor physical condition before the operation may constitute a risk factor for adverse functional outcome.

In the last cluster, the contribution of several psychological variables was tested. For all outcome variables except increased functional limitations, psychological variables added to the predictive power of the model. In line with studies on acute postoperative pain and recovery ${ }^{17-20}$, fear of surgery proved to be the most consistent psychological predictor of unfavourable outcome. Patients who where fearful of the long-term consequences of the operation had an increased chance of more pain and poor recovery at FU. In addition, fear had a negative influence on the improvement of QOL 6 months after the operation. Pain catastrophizing was recently identified as an important predictor of acute postoperative pain ${ }^{21-22}$, but it did not turn up as a risk factor for long-term increases in pain or funtional limitations in the present study. 


\section{CHAPTER 5}

Probably the surgical fear questionnaire more closely taps the various concerns of patients undergoing an operation than the pain catastrophizing scale which focusses uniquely on the pain experience.

Various studies have reported that optimism promotes recovery after surgical intervention. ${ }^{25}$ We found that an optimistic attitude did not protect against increased pain or increased limitations in physical functioning 6 months after surgery, but that it did influence patients' overall estimates of their recovery and QOL at follow-up. Optimistic people may be more inclined to rate their recovery as satisfactory independent of the level of pain and limitations they experience. Moreover, they may be more resistent to the negative influence of pain and limitations on their QOL.

A limitation of the present study concerns the exclusion of patients who experienced complications during surgery and patients who were transferred to the intensive care unit because they needed extended ventilatory support. Due to practical constraints, these patients could not be approached for immediate postoperative assessment. In addition, patients declining participation were more often in bad physical condition preoperatively (i.e. ASA grade III). This may limit the generalizability of the findings since there may have been a selective drop-out of patients most at risk for long-term unfavourable outcome. Prevalence of unfavourable outcome may thus be higher than we report in the present study. However, in spite of this potential underestimation, predictors of unfavourable outcome could be indentified.

In conclusion, we identified several somatic and psychological predictors of longterm unfavourable outcome. The most important somatic predictors were operations with a duration longer than 3 hours and high acute postoperative pain. Although we should be cautious with a causal interpretation of the results, the implication of this could be that adequate treatment of acute postoperative pain may reduce the risk of long-term adverse outcome. Furthermore, patients who are in a bad physical condition preoperatively (ASA grade III) may require extra attention postoperatively as they are at risk for further deterioration of their condition. The most important psychological predictor appeared to be fear of surgery. Identification of patients with high levels of fear and subsequently reducing these fears may promote recovery and reduce the risk of adverse outcome. Interventions to better prepare people for surgery have been found to be able to reduce patients' fear, reduce postoperative pain and to aid recovery ${ }^{39-40}$. Clearly, more research into somatic and psychological interventions to prevent long-term unfavourable outcomes of surgery is warranted.

\section{Acknowledgements}

This study was supported by a grant from the Dutch Foundation of Scientific Research (Zon-MW), grant \# 110000007. 


\section{REFERENCES}

1. Macrae WA. Chronic pain after surgery. Br J Anaesth 2001; 87:88--98.

2. Perkins FM, Kehlet $\mathrm{H}$. Chronic pain as an outcome of surgery. A review of predictive factors. Anesthesiology 2000; 93:1123-1133.

3. Kehlet, $H$, Jensen, TS, Woolf, CJ. Persistent postsurgical pain: risk factors and prevention. Lancet 2006; $367: 1618-1625$

4. Bruce J, Drury N, Poobalan AS, et al. The prevalence of chronic chest and leg pain following cardiac surgery: a historical cohort study. Pain 2003; 104:265--273.

5. Eisenberg $E$, Pultorak $Y$, Pud D, et al. Prevalence and characteristics of post coronary artery bypass graft surgery pain (PCP). Pain 2001; 92:11-17.

6. Smith WC, Bourne D, Squair J, et al. A retrospective cohort study of post mastectomy pain syndrome. Pain 1999; 83:91--95.

7. Wallace MS, Wallace AM, Lee J, et al. Pain after breast surgery: a survey of 282 women. Pain 1996 66:195--205.

8. Kumar S, Wilson RG, Nixon SJ, et al. Chronic pain after laparoscopic and open mesh repair of groin hernia. Br J Surg 2002; 89:1476-1479.

9. Callesen T. Bech K, Kehlet H. Prospective study of chronic pain after groin hernia repair. $\mathrm{Br} \downarrow \mathrm{J}$ Surg 1999; 86:1528-1531.

10. Poobalan AS, Bruce J, King $P M$, et al. Chronic pain and quality of life following open inguinal hernia repair. Br J Surg 2001; 88:1122-1126.

11. Poobalan AS, Bruce J, Smith WC, et al. A review of chronic pain after inguinal herniorrhaphy. Clin J Pain 2003:19:48-54.

12. Cunningham J, Temple WJ, Mitchell $P$, et al. Cooperative hernia study. Pain in the postrepair patient. Ann Surg 1996; 224:598--602.

13. Coderre $T J$, Katz J, Vaccarino $A L$, et al. Contribution of central neuroplasticity to pathological pain: review of clinical and experimental evidence. Pain 1993; 52:259--285.

14. Katz $J$, Jackson $M$, Kavanagh $B P$, et al. Acute pain after thoracic surgery predicts long-term postthoracotomy pain. Clin J Pain 1996; 12:50--55.

15. Hannan EL, Magaziner J, Wang JJ, et al. Mortality and locomotion 6 months after hospitalization for hip fracture: risk factors and risk-adjusted hospital outcomes. JAMA 2001; 285:2736-2742.

16. Orbell $S$, Johnston $M$, Rowley $D$, et al. Self-efficacy and goal importance in the prediction of physical disability in people following hospitalization: A prospective study. Br J Health Psychol 2001; 6:25--40.

17. Taenzer $P$, Melzack $R$, Jeans ME. Influence of psychological factors on postoperative pain, mood and analgesic requirements. Pain $1986 ; 24: 331-342$.

18. Munafo MR, Stevenson J. Anxiety and surgical recovery. Reinterpreting the literature. J Psychosom Res 2001; 51:589--596.

19. Caumo W, Schmidt AP, Schneider $C N$, et al. Preoperative predictors of moderate to intense acute postoperative pain in patients undergoing abdominal surgery. Acta Anaesthesiol Scand 2002; 46:1265--1271.

20. Kalkman CJ, Visser K, Moen J, et al. Preoperative prediction of severe postoperative pain. Pain 2003; 105:415-423.

21. Pavlin DJ, Sullivan MJ, Freund $P R_{1}$ et al. Catastrophizing: a risk factor for postsurgical pain. Clin $J$ Pain 2005; $21: 83-\cdots 90$

22. Granot $M$, Ferber $S G$. The roles of catastrophizing and anxiety in the prediction of postoperative pain intensity. A prospective study. Clin J Pain 2005; 21:439 -445

23. Kendell $K$, Saxby $B$, Farrow $M$, et al. Psychological factors associated with short-term recovery from total knee replacement. Br J Health Psychol 2001; 6:41--52

24. Scheier MF, Matthews KA, Owens JF, et al. Optimism and rehospitalization after coronary artery bypass graft surgery. Arch Intern Med 1999; 159:829--835.

25. Scheier MF, Matthews KA, Owens JF, et al. Dispositional optimism and recovery from coronary artery bypass surgery: the beneficial effects on physical and psychological well-being. J Pers Soc Psychol $1989 ; 57: 1024-1040$.

26. Waldrop D, Lightsey $O$, Ethington $C$. Self-efficacy, optimism, health competence, and recovery from orthopedic surgery. J Counsel Psychol 2001; 48:233-238.

27. Bowley DM, Butler $M$, Shaw $S$, et al. Dispositional pessimism predicts delayed return to normal activities after inguinal hernia operation. Surgery $2003 ; 133: 141-146$. 


\section{CHAPTES}

28. King KB, Rowe MA, Kimble LP, et al. Optimism, coping and long-term recovery from coronary artery surgery in women. Res Nurs Health 1998; 21:15--26.

29. Orbell $S$, Johnston $M$, Rowley $D$, et al. Cognitive representations of illness and functional and affective adjustment following surgery for osteoarthritis. Soc Sci Med 1998; 47:93--102.

30. Sullivan MJL, Bishop SR, Pivik J. The Pain Catastrophizing Scale: Development and validation. Psychol Assessment 1995; 7:524--532.

31. Carver CS, White TL. Behavioral inhibition, behavioral activation, and affective responses to impending reward and punishment: The BIS/BAS Scales. J Pers Soc Psychol 1994; 67:319--333.

32. Scheier MF, Carver CS, Bridges MW. Distinguishing optimism from neuroticism (and trait anxiety, selfmastery, and self-esteem): A reevaluation of the Life Orientation Test. J Pers Soc Psychol 1994;
67:1063-1078.

33. Jerusalem M, Schwarzer R. Self-efficacy as a resource factor in stress apparaisal processes. In: Schwarzer R, ed. Self-effcacy: Thought control of action. Washington DC, USA:Hemisphere; 1992: 195213.

34. Rand Health Science Program. Rand 36-item Health Survey Manual. In: Rand, ed. Santa Monica, 1992.

35. Koivula M, Tarkka MT, Tarkka M, et al. Fear and anxiety in patients at different time-points in the coronary artery bypass process. Int $J$ Nurs Stud 2002; 39:811--822.

36. Rawal N. Organization, function, and implementation of acute pain service. Anesthesiol Clin North America 2005; 23:211--255.

37. Kleinbeck SV. Self-reported at-home postoperative recovery. Res Nurs Healt $2000 ; 23: 461-472$

38. Stubhaug A. Can opioids prevent postoperative chronic pain? Eur J Pain 2005; 9:153156.

39. Gammon J, Mulholland $\mathrm{CW}$. Effect of preparatory information prior to elective total hip replacement on psychological coping outcomes. J Adv Nurs 1996; 24:303--308.

40 Lin LY, Wang RH. Abdominal surgery, pain and anxiety: preoperative nursing intervention. J Adv Nurs 2005; 51:252--260. 


\section{Chapter 6}

\section{Predictors of unfavourable outcome after surgical intervention: A 12-month follow-up study}

Madelon L. Peters PhD, Micha Sommer MD, Maarten van Kleef MD PhD \& Marco A.E. Marcus MD PhD

This study was supported by a grant from the Dutch Foundation of Scientific Research (Zon-MW), grant \# 110000007

Submitted to New England Journal of Medicine

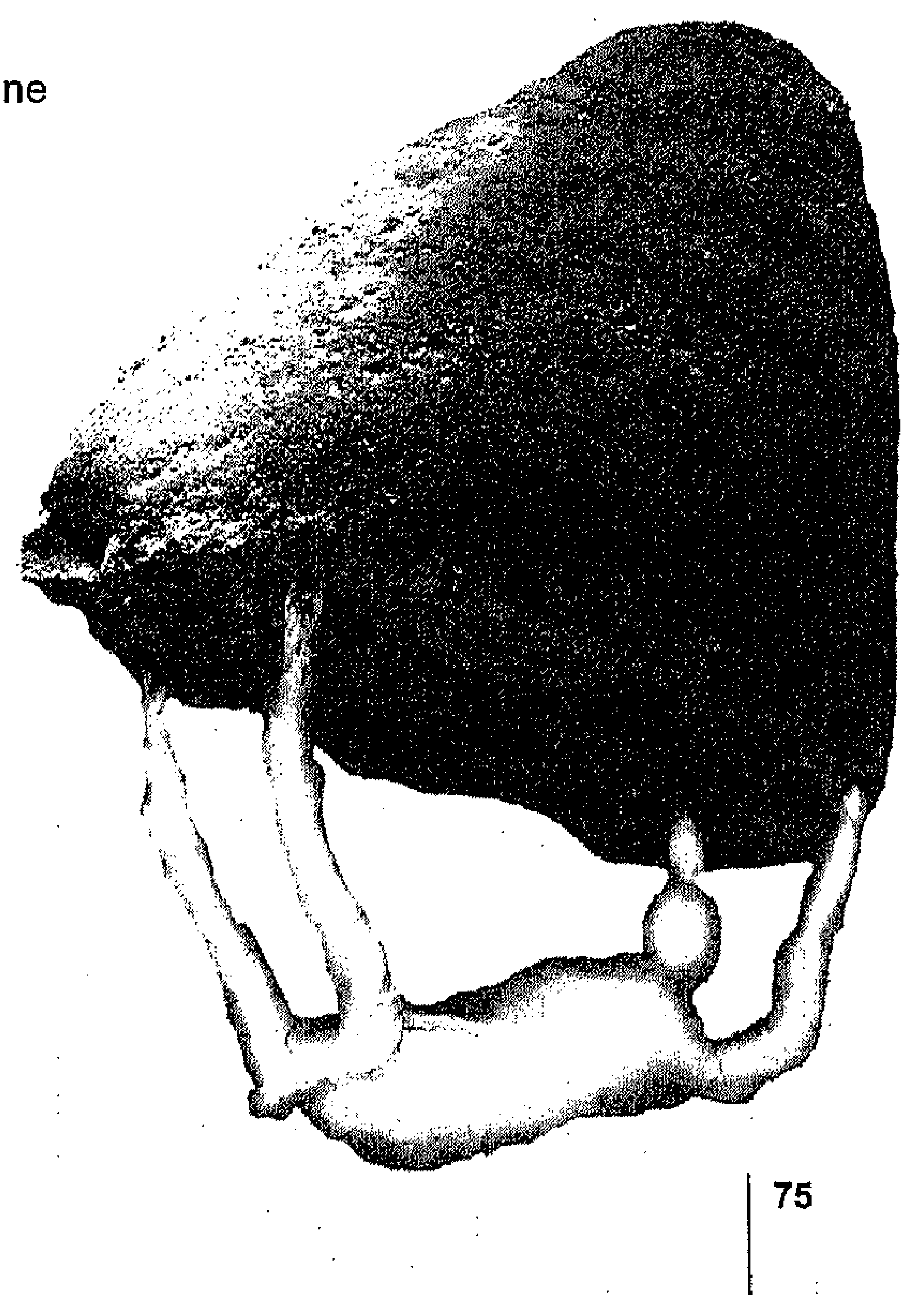




\section{CHAPTER 6}

\section{ABSTRACT}

Objective: To examine whether the somatic and psychological predictors that we previously identified for increased pain, functional limitations and global perceived recovery six months after surgery are robust and still predict adverse outcome at 12 months follow-up.

Methods: Surgical patients that had participated in our previous prospective cohort study on adverse outcome 6 months after their operation were approached again at 12 months. All patients had completed psychological questionnaires preoperatively and acute postoperative pain scores were obtained until 4 days after the operation. The follow-up questionnaires at 6 and 12 months assessed pain, functional limitations and global perceived recovery. 12-Months follow-up data were available for 401 patients. Multivariable logistic and linear regression analyses were used to identify predictors of longterm adverse outcomes.

Results: Confirming our previous results, the most important predictors of unfavourable outcome were duration of the operation and high levels of acute postoperative pain. Patients reporting high levels of pain 4 days after the operation and patients undergoing an operation of longer than 3 hours were at risk of increased pain, increased functional limitations and lower levels of global perceived recovery 12 months after the operation. We also confirmed the role of preoperative fear of surgery and pain catastrophizing, especially in relation to functional outcomes (i.e., limitations and global recovery). Patients reporting adverse outcome at 12 months only (new cases) appeared to be different form patients with persistent complaints: their complaints were not predicted by the same somatic or psychological variables.

Conclusions: The 12 months follow-up data confirm the results of the analyses of adverse outcome at 6 months post-operation. By and large the same predictors emerged, attesting to the validity and robustness of these findings. 


\section{INTRODUCTION}

Chronic pain is an undesirable but not uncommon consequence of surgical intervention $^{1-2}$. In a previous paper we presented the 6 months follow-up data of 625 patients undergoing various elective surgical procedures ${ }^{3}$. We found that $12 \%$ of patients reported that their pain had become worse or that they had developed a new pain complaint due to the operation, and $17 \%$ reported a detrimental effect on their functional abilities. Several predictors of adverse outcome were identified. Patients reporting high levels of acute postoperative pain and patients undergoing an operation of longer than 3 hours were at risk of increased pain, increased functional limitations and poor global recovery. Moreover we also found psychological variables to influence outcome at 6 months. Preoperative surgical fear was associated with more pain and poor global recovery 6 months later. A global optimistic attitude about life was associated with better recovery.

The present paper reports on the 12 months follow-up. Patients were approached again 12 months following their operation and filled in an identical set of questionnaires. We studied the persistence of complaints and verified whether the predictors of adverse outcome that we found at 6 months are still associated with outcome at 12 months. In addition, several new cases of adverse outcome occurred at the 12 months follow-up assessment and we examined whether these delayed complaints can be predicted from the same pre- and peri-operative factors, or whether they originate from other causes. Thus, the aim of this study is to establish the robustness of the previously identified predictors of outcome and the generalizability of these predictors in the explanation of future complaints.

\section{METHODS}

\section{Patients}

The follow-up study was approved by the Ethical Committee of the University Hospital Maastricht, The Netherlands. All patients had undergone elective surgery at the University Hospital Maastricht between February 2003 and August 2003 and participated in our previous outcome study. At the time of the first follow-up, patients were asked for consent to be approached again 12 months after surgery. A total of 619 of the 625 patients in the first follow-up $(99 \%)$ agreed to participate in the second followup. Patients with malignancies $(n=72)$ were excluded form further participation and a total of 547 questionnaires were send out, 452 of which were returned (response rate: $83 \%$ ). A further 51 patients were excluded because they had a new operation during the previous 6 months, leaving 401 participants for the analyses. Mean age of the patients included in the analyses was 55 year (range 19-88) and $54 \%$ was female. 


\section{CHA}

\section{Assessment}

Preoperative assessment included the Pain catastrophizing scale (PCS) ${ }^{4}$, the Life Orientation Test (LOT; dispositional optimism) ${ }^{5}$ and a questionnaire on surgical anxiety (with two subscales: fear of the immediate consequences of the operation and fear of the longterm consequences) ${ }^{3}$. Health-related quality of life was measured using the Medical Outcomes Study Short-Form General Health Survey (SF-36) ${ }^{6}$. More details on these questionnaires can be found in our previous paper ${ }^{3}$.

Operations were catagorized into three groups based on the anticipated level of postoperative pain (minor, intermediate and major) ${ }^{3}$ and according to the anatomical region of the body involved (head/neck, upper extremities, thorax-noncardiac, abdominal high, abdominal low, abdominal high and low, lower extremities, back, multiple sites). Type of anaesthesia was coded as general, locoregional or general plus locoregional. Duration of the operation was dichotomized as $<3$ or $\geq 3$ hours. Postoperative pain intensity at rest was scored three times a day during the first 4 days on a $100 \mathrm{~mm}$ VAS. Following our previous study we use the average postoperative pain intensity on day 4 in all analyses.

At 12 months, participants were sent the long-term recovery questionnaire and the SF-36 by mail. These questionnaires were identical to the ones used at 6 months follow-up. Following our previous study, the main outcome variables were (1) Increased pain due to the operation (dichotomous; derived from the long-term recovery questionnaire) (2) Increased functional limitations due to the operation (dichotomous; derived from the long-term recovery questionnaire) and (3) the Global Surgical Recovery (GSR) index ${ }^{7}$ ("If $100 \%$ recovery is back to the usual health you had before you got sick and had surgery, what percent of recovery you are at now?"). Additionally we looked at the pain subscale of the SF-36.

\section{Statistical analyses}

Logistic regression analysis was used to identify the factors that were associated with the dichotomous outcome variables increased pain due to the operation and increased functional limitations. As potential predictors we examined demographic variables (age, sex), surgery related variables (type of intervention, type of anesthesia), somatic variables (preoperative pain, ASA status, acute postoperative pain) and psychological variables (surgical fear, pain catastrophizing and optimism). Acute postoperative pain was dichotomized according to VAS $\geq 40 \mathrm{~mm}$ and psychological variables were dichotomized using median split. In order to reduce the number of predictors we first conducted univariate analyses, using t-tetst for continuous and $x^{2}$ analyses for categorical variables. Variables that differentiated between patients with or without persistent adverse outcome in the univariate analyses were subsequently included in the logistic regression analyses. All variables were entered with a forward procedure (likelihood ratio), using a criterium of $p<0.05$ for entering variables in the model. 
Linear regression analysis with blockwise entry of independent variables was used to analyze the continuous variables global perceived recovery and the pain subscale of the SF36. In the first block we entered the surgery related and demographic variables (type of operation, location, sex and age) and for the analyses of SF-36 pain, also the preoperative SF-36 pain score. In the second block we entered the somatic predictors ASA status, duration of the operation, type of anesthesia and acute postoperative pain. In the final block the psychological predictors were entered. Block 2 and 3 used a forward entry procedure with $p<0.0 .5$ as the criterium for adding a variable. For the linear regression analysis, the continuous quality of the acute postoperative pain score and the psychological variables was retained. Analyses were performed with the Statistical Package for Social Sciences (SPSS)-version 11.5.

\section{RESULTS}

\section{Persistent pain at follow-up}

35 patients $(9 \%)$ reported that due to the operation they experienced more or new pain at 12 months follow-up. $16(4 \%)$ of these already had pain at 6 months (persistent cases) while $19(5 \%)$ were new cases. In 20 patients that had reported pain at 6 months the pain had resolved at 12 months. The 16 patients with persistent postoperative pain were compared to patients who were painfree at both assessment times $(n=346)$. The two groups differed significantly on the variables type of operation, duration of operation, anesthetic technique, acute postoperative pain and on the two subscales of the preoperative surgical fear questionnaire. Table 1 shows the results of the univariate analyses. The significant predictors from the univariate analyses were used in the subsequent multivariate logistic regression analyses. Two predictors emerged: patients with an operation longer than 3 hours and patients with high levels of postoperative pain had a higher chance of developing post-surgical pain that persisted at 12 months follow-up (see table 2 for the odds ratios and confidence intervals).

Since these analyses were based on a small sample of positive cases, we also analyzed the data in another way, using the continuous pain measure from the SF36. The score on the SF36 pain subscale increased from 62.4 before the operation to 73.7 at 6 months, and 74.8 at 12 months post-operation, where higher scores denote better quality of life and thus less pain. The most prominent predictor of the SF-36 pain score at 12 months was the preoperative SF-36 pain score. Demographic and surgery related variables did not contribute to the 12 months pain score. Confirming the logistic regression analysis, the duration of surgery and pain intensity 4 days after the operation were significantly related to higher pain scores at 12 months. In addition, ASA status was predictive of outcome, with status $I I$ and III being associated with more pain at 12 months than ASA status I. Table 3 shows the final model. 
CMATESE

Table 1: Univariate tests of differences in independent variables for patients pain free during both follow-up periods and patients with persistent pain.

\begin{tabular}{|c|c|c|c|}
\hline & $\begin{array}{l}\text { Pain free } \\
(n=346)\end{array}$ & $\begin{array}{l}\text { Persistent Pain } \\
(n=16)\end{array}$ & \\
\hline Mean age & 54.8 & 50.5 & $\mathrm{t}=0.9, \mathrm{p}=0.37$ \\
\hline $\begin{array}{l}\text { Sex } \\
\text { Male } \\
\text { Female }\end{array}$ & $\begin{array}{l}160(46 \%) \\
186(54 \%)\end{array}$ & $\begin{array}{l}7(44 \%) \\
9(56 \%)\end{array}$ & $x^{2}=0.4 ; p=0.85$ \\
\hline $\begin{array}{l}\text { Type of operation } \\
\text { minor } \\
\text { intermidiate \& major }\end{array}$ & $\begin{array}{l}123(35 \%) \\
223(65 \%)\end{array}$ & $\begin{array}{r}2(12 \%) \\
14(88 \%)\end{array}$ & $X^{2}=4.2 ; p=0.04$ \\
\hline $\begin{array}{l}\text { Duration of operation } \\
<3 \mathrm{hrs} \\
\geq 3 \mathrm{hrs}\end{array}$ & $\begin{array}{c}317(92 \%) \\
29(8 \%)\end{array}$ & $\begin{array}{r}11(69 \%) \\
5(31 \%)\end{array}$ & $x^{2}=6.4 ; p=0.01$ \\
\hline $\begin{array}{l}\text { Preoperative pain } \\
\text { No } \\
\text { Yes }\end{array}$ & $\begin{array}{l}139(40 \%) \\
207(60 \%)\end{array}$ & $\begin{array}{r}5(31 \%) \\
11(69 \%)\end{array}$ & $X^{2}=0.5 ; p=0.47$ \\
\hline $\begin{array}{l}\text { Anesthetic technique } \\
\text { General } \\
\text { General + locoregional } \\
\text { Locoregional }\end{array}$ & $\begin{array}{l}243(70 \%) \\
27(8 \%) \\
76(22 \%)\end{array}$ & $\begin{array}{c}15(94 \%) \\
1(6 \%) \\
0(0 \%)\end{array}$ & $x^{2}=8.0 ; p=0.02$ \\
\hline $\begin{array}{l}\text { ASA status } \\
1 \\
\text { II } \\
\text { III }\end{array}$ & $\begin{array}{c}169(49 \%) \\
148(43 \%) \\
29(8 \%)\end{array}$ & $\begin{array}{l}5(31 \%) \\
8(50 \%) \\
3(19 \%)\end{array}$ & $X^{2}=2.8 ; p=0.26$ \\
\hline $\begin{array}{c}\text { Pain on day } 4 \\
\text { Pain }<40 \\
\text { Pain } \geq 40\end{array}$ & $\begin{array}{c}322(93 \%) \\
24(7 \%)\end{array}$ & $\begin{array}{r}11(69 \%) \\
5(31 \%)\end{array}$ & $X^{2}=7.8 ; p<0.01$ \\
\hline $\begin{array}{l}\text { Surgical fear (immediate) } \\
\text { Low } \\
\text { High }\end{array}$ & $\begin{array}{l}172(50 \%) \\
174(50 \%)\end{array}$ & $\begin{array}{c}3(19 \%) \\
13(81 \%)\end{array}$ & $x^{2}=6.4 ; p=0.01$ \\
\hline $\begin{array}{l}\text { Surgical fear (longterm) } \\
\text { Low } \\
\text { High }\end{array}$ & $\begin{array}{l}181(52 \%) \\
165(48 \%)\end{array}$ & $\begin{array}{r}4(25 \%) \\
12(75 \%)\end{array}$ & $x^{2}=4.8 ; p=0.03$ \\
\hline $\begin{array}{l}\text { Pain catastrophizing } \\
\text { Low } \\
\text { High }\end{array}$ & $\begin{array}{l}187(54 \%) \\
159(46 \%)\end{array}$ & $\begin{array}{l}7(44 \%) \\
9(56 \%)\end{array}$ & $x^{2}=0.7 ; p=0.42$ \\
\hline $\begin{array}{l}\text { Optimism } \\
\text { Low } \\
\text { High }\end{array}$ & $\begin{array}{l}148(44 \%) \\
192(56 \%)\end{array}$ & $\begin{array}{l}7(47 \%) \\
8(53 \%)\end{array}$ & $X^{2}=0.6 ; p=0.81$ \\
\hline
\end{tabular}


Table 2. Results of the logistic regression analysis for increased pain and increased functional limitations at 12-months follow-up. Only variables significant in the univariate analyses were included as predictors.

\begin{tabular}{|c|c|c|c|c|c|c|}
\hline \multirow[t]{2}{*}{ Independent variable } & \multirow[b]{2}{*}{$N$} & \multicolumn{2}{|c|}{ Increased Pain at 12 months } & \multicolumn{2}{|c|}{$\begin{array}{l}\text { Increased Functional } \\
\text { Limitations at } 12 \text { months }\end{array}$} & \multirow[b]{2}{*}{$P$} \\
\hline & & OR $(95 \% \mathrm{Cl})$ & $P$ & $N$ & OR $(95 \% \mathrm{Cl})$ & \\
\hline \multicolumn{7}{|l|}{ Duration of Surgery } \\
\hline$<3$ hours & 328 & 1.00 & & 323 & 1.00 & \\
\hline$\geq 3$ hours & 34 & $4.77(1.49-15.25)$ & 0.01 & 26 & $4.67(1.64-13.29)$ & 0.001 \\
\hline \multicolumn{7}{|l|}{ Pain on day 4} \\
\hline Pain $<40$ & 333 & 1.00 & & 319 & 1.00 & \\
\hline Pain $\geq 40$ & 29 & $5.87(1.82-18.90)$ & 0.01 & 30 & $3.21(1.16-8.84)$ & 0.02 \\
\hline \multicolumn{7}{|l|}{ ASA status } \\
\hline 1 & & & & 163 & 1.00 & \\
\hline$\|$ & & & & 151 & $3.68(1.14-10.59)$ & 0.01 \\
\hline III & & & & 35 & $6.83(1.98-23.59)$ & 0.01 \\
\hline \multicolumn{7}{|l|}{ Long-term fear } \\
\hline Low $(<10)$ & & & & 179 & 1.00 & \\
\hline High $(\geq 10)$ & & & & 170 & $3.14(1.30-7.62)$ & 0.01 \\
\hline
\end{tabular}

Table 3. Results of the linear regression analysis for the SF-36 pain subscale score at 12-months follow-up. Only the final model is shown.

\begin{tabular}{|c|c|c|}
\hline & Standardized B & $\mathrm{P}$ \\
\hline Preoperative SF-36 pain score & 0.411 & 0.001 \\
\hline \multicolumn{3}{|l|}{ Type of operation ${ }^{1}$} \\
\hline Intermediate & 0.036 & 0.57 \\
\hline Major & -0.054 & 0.47 \\
\hline \multicolumn{3}{|l|}{ Body region ${ }^{2}$} \\
\hline Upper extremities & -0.038 & 0.45 \\
\hline Thorax-noncardiac & -0.073 & 0.12 \\
\hline Abdominal High & 0.000 & 0.99 \\
\hline Abdominal Low & 0.016 & 0.78 \\
\hline Abdominal $H+L$ & 0.100 & 0.13 \\
\hline Lower extremities & -0.027 & 0.70 \\
\hline Back & -0.070 & 0.24 \\
\hline Multiple sites & -0.090 & 0.05 \\
\hline Age & 0.003 & 0.95 \\
\hline Sex & -0.008 & 0.87 \\
\hline Duration of Surgery & -0.117 & 0.02 \\
\hline Pain intensity day 4 & -0.117 & 0.02 \\
\hline \multicolumn{3}{|l|}{ ASA status $^{3}$} \\
\hline ASA grade $\|$ & -0.123 & 0.01 \\
\hline ASA grade II! & -0.179 & 0.001 \\
\hline
\end{tabular}

$R^{2}=0.34, F(367.17)=10.4 p<0.001$

${ }^{1}$ Reference category: minor

${ }^{2}$ Reference category : head/neck

${ }^{3}$ Reference category: ASA grade I 


\section{Increased functional limitations at follow-up}

63 patients $(16 \%)$ reported that due to the operation they felt more limited in their functioning at 12 months follow-up than before the operation. In 30 patients $(8 \%)$ these functional limitations were already present at 6 months while $33(8 \%)$ were new cases. Nineteen patients that had reported to experience increased functional limitations as 6 months, did not report this anymore at 12 months. The 30 patients with persistent functional limitations were compared with patients that reported no limitations at either assesment time $(n=319)$. Univariate analyses showed that patients with persistent functional limitations significantly differed from those without limitations on the variables type of operation (intermediate and major vs. minor), duration of operation, ASA status, acute postoperative pain, preoperative surgical fear (longterm consequences) and preoperative pain catastrophizing (table 4). The subsequent multivariate logistic regression analyses yielded four significant predictors: a higher preoperative ASA status, operations longer than 3 hours, a high level of acute postoperative pain and fear of the longterm consequences of the operation all increased the chance of persistent functional limitations (table 2). Preoperative pain catastrophizing was also significantly related to persistent functional limitations when it was included in the model instead of surgical fear (OR, 2.99;95\% Cl, 1.25-7.16).

\section{Global perceived surgical recovery}

Global perceived recovery (GSR) increased form 79.3 at 6 months to 82.2 at 12 months $(t=-3.11, p=.002)$. The GSR score at 12 months was analyzed with blockwise linear regression analyses. The final model is shown in table 5 . The variables duration of the operation and acute postoperative pain on day 4 were significantly and negatively associated with global perceived recovery. When entered simultaneously, the only psychological predictor remaining in the final model was pain catastrophizing. Higher pain catastrophizing before the operation was related to worse perceived recovery. When pain catastrophizing in the final model was substituted by longterm surgical fear, this proved to be a significant predictor as well $(\beta=-.10, p=-.045)$, and so did optimism (in a positive direction; $\beta=.11, p=-.029$ ).

\section{Predictors for new cases}

To examined whether the same predictors of adverse outcome emerged for patients with new complaints of increased pain or functional limitations at 12 months, the same multivariate logistic regression analysis as reported above were performed, but now with new cases instead of persistent cases compared against pain-free patients. The two predictors of increased pain in patients with persistent pain, duration of the operation and acute postoperative pain, did not significantly predict new pain cases. Of the four predictors of increased functional limitation in patients with increased complaints, only duration of the operation also predicted new cases of increased functional limitations $(\mathrm{OR}, 6.02 ; 95 \% \mathrm{Cl}, 2.38-15.2)$. It seems that patients with new 
comlaints of pain or functional limitations form a distinct group for which the complaints are not predicted by the same pre- and postoperative variables predicting persistent postoperative pain. Therefore, we also explored via univariate analyses whether any of the other variables included in the study were predictive of new complaints. Neither for new pain nor for new functional limitations at 12 months other predictors were identified.

Table 4: Univariate tests of differences in independent variables for patients without fuctional limiations during both follow-up periods and patients with persistent functional limitations.

\begin{tabular}{|c|c|c|c|}
\hline & $\begin{array}{l}\text { No limitations } \\
(n=319)\end{array}$ & $\begin{array}{l}\text { Persistent limitations } \\
(n=30)\end{array}$ & \\
\hline Mean age & 53.6 & 57.1 & $t=-1.3, p=0.20$ \\
\hline \multicolumn{4}{|l|}{ Sex } \\
\hline Male & $149(47 \%)$ & $14(47 \%)$ & $x^{2}=0.0 ; p=1.00$ \\
\hline Female & $170(53 \%)$ & $16(53 \%)$ & \\
\hline \multicolumn{4}{|l|}{ Type of operation } \\
\hline Minor & $113(35 \%)$ & $5(17 \%)$ & $X^{2}=4.8 ; p=0.03$ \\
\hline Intermediate \& major & $206(65 \%)$ & $25(83 \%)$ & \\
\hline \multicolumn{4}{|l|}{ Duration of operation } \\
\hline$<3 \mathrm{hrs}$ & $300(94 \%)$ & $23(77 \%)$ & $x^{2}=8.4 ; p<0.01$ \\
\hline$\geq 3 \mathrm{hrs}$ & $19(6 \%)$ & $7(23 \%)$ & \\
\hline \multicolumn{4}{|l|}{ Preoperative pain } \\
\hline No & $184(58 \%)$ & $20(67 \%)$ & $x^{2}=0.9 ; p=0.33$ \\
\hline Yes & $135(42 \%)$ & $10(33 \%)$ & \\
\hline \multicolumn{4}{|l|}{ Anesthetic technique } \\
\hline General & $231(72 \%)$ & $21(70 \%)$ & $x^{2}=0.2 ; p=0.89$ \\
\hline General + locoregional & $24(8 \%)$ & $3(10 \%)$ & \\
\hline Locoregional & $64(20 \%)$ & $6(20 \%)$ & \\
\hline \multicolumn{4}{|l|}{ ASA status } \\
\hline 1 & $157(49 \%)$ & $6(20 \%)$ & $X^{2}=11.9 ; p=<0.01$ \\
\hline$\|$ & $134(42 \%)$ & $17(57 \%)$ & \\
\hline III & $28(9 \%)$ & $7(23 \%)$ & \\
\hline \multicolumn{4}{|l|}{ Pain on day 4} \\
\hline Pain < 40 & $296(93 \%)$ & $23(77 \%)$ & $X^{2}=6.7 ; p=0.01$ \\
\hline Pain $\geq 40$ & $23(7 \%)$ & $7(23 \%)$ & \\
\hline \multicolumn{4}{|l|}{ Surgical fear (immediate) } \\
\hline Low & $162(51 \%)$ & $10(33 \%)$ & $X^{2}=3.4 ; p=0.07$ \\
\hline High & $157(49 \%)$ & $20(67 \%)$ & \\
\hline \multicolumn{4}{|l|}{ Surgical fear (longterm) } \\
\hline Low & $171(54 \%)$ & $8(27 \%)$ & $X^{2}=8.2 ; p<0.01$ \\
\hline High & $148(46 \%)$ & $22(73 \%)$ & \\
\hline \multicolumn{4}{|l|}{ Pain catastrophizing } \\
\hline Low & $175(55 \%)$ & $9(30 \%)$ & $x^{2}=6.9 ; p<0.01$ \\
\hline High & $144(45 \%)$ & $21(70 \%)$ & \\
\hline \multicolumn{4}{|l|}{ Optimism } \\
\hline Low & $135(42 \%)$ & $15(50 \%)$ & $X^{2}=0.7 ; p=0.42$ \\
\hline High & $184(58 \%)$ & $15(50 \%)$ & \\
\hline
\end{tabular}




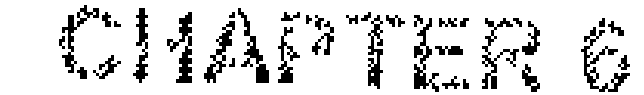

Table 5. Results of the linear regression analysis for Global Surgical Recovery at 12-months follow-up. Only the final model is shown.

\begin{tabular}{|c|c|c|}
\hline & Standardized B & $\mathrm{P}$ \\
\hline \multicolumn{3}{|l|}{ Type of operation ${ }^{1}$} \\
\hline Intermediate & -0.053 & 0.92 \\
\hline Major & -0.007 & 0.42 \\
\hline \multicolumn{3}{|l|}{ Body region ${ }^{2}$} \\
\hline Upper extremities & -0.122 & 0.02 \\
\hline Thorax-noncardiac & 0.007 & 0.89 \\
\hline Abdominal High & 0.065 & 0.23 \\
\hline Abdominal Low & 0.064 & 0.28 \\
\hline Abdominal $H+L$ & 0.044 & 0.51 \\
\hline Lower extremities & -0.091 & 0.19 \\
\hline Back & -0.031 & 0.61 \\
\hline Multiple sites & -0.104 & 0.04 \\
\hline Age & -0.057 & 0.25 \\
\hline Sex & -0.096 & 0.06 \\
\hline Duration of Surgery & -0.159 & 0.01 \\
\hline Pain intensity day 4 & -0.197 & 0.001 \\
\hline Pain catastrophizing & -0.236 & 0.001 \\
\hline
\end{tabular}

\section{DISCUSSION}

In our previous paper, we reported that $12 \%$ of patients undergoing various types of surgery indicated that due to surgery they had more pain 6 months after the operation than they had before the operation and $17 \%$ indicated that they experienced more functional limitations. The present paper looked at the 12 months follow-up data and found that the percentage of patients still reporting increased pain at 12 months follow-up had decreased to $4 \%$ and the percentage still experiencing more limitations to $8 \%$. It should be noted that there seems to have been selective drop out. When looking at the 6 months reports of adverse outcome in the present sample, $9 \%$ of the patients reported to have more pain and $12 \%$ to have more limitations than before the operation. The decrease in these percentages may be due to our exclusion of patients with malignancy and patients that had had a second operation.

It is also apparent from these numbers that a substantial part of patients reporting an adverse outcome of surgery at 6 months follow-up, had recovered by 12 months post-operation. Some new cases also occurred, but it may be questioned whether the complaints of these patients can actually be attributed to the operation. 
The predictors that were previously identified for adverse outcome at 6 months and were now confirmed for persistent complaints did not bear out in patients with increased pain and limitations at 12 months only, suggesting that this is in fact a distinct group. This urges for caution in interpreting findings from studies using a single assessment of adverse surgical outcome after a prolonged period of time. Even though patients themselves may attribute their present complaints to the operation, our data suggests that this may not actually be the case.

The present results are consistent with the results reported in our previous paper on the outcomes at 6 months follow-up. Again acute postoperative pain and duration of the operation were identified as major predictors of persistent pain, persistent functional limitations and global perceived recovery. For the SF-36 pain score and increased functional limitations, ASA status was identified as an additional predictor. In our analyses of the 6 months follow-up, we also found ASA to be related to functional recovery at follow-up. It should be noted that the pain subscale of the SF-36 consists of 2 items, one asking about pain intensity the other about interference by pain and therefore also includes this element of functional limitations.

The role of psychological variables was also confirmed. Fear of the longterm consequences of the operation was significantly related to persistent functional limitations and global perceived recovery in the multivariate analyses. For increased pain, only the univariate association with fear of the longterm consequences of the operation reached significance. It should also be noted that on simultaneous entry of surgical fear and pain catastrophizing in the prediction of perceived recovery, only pain catastrophizing reached significance. Previously we found that for the 6 months outcomes also pain catastrophizing and fear of the longterm consequences were both predictive when entered separately. With simultaneous entry only one predictor remains in the model, probably because there is conceptual overlap between the constructs. In the present sample, fear of the longterm consequences and pain catastrophizing were indeed significantly, although moderately $(r=0.38)$, correlated with each other. The previously found contribution of optimism was sustained for perceived recovery, but again only when entered separately from pain catastrophizing. Optimism may indeed protect against adverse outcomes, but its contribution seems to be modest.

One other predictor that appeared associated with persistent pain in the univariate analyses should be mentioned. Only one of the 16 patients with persistent pain had had locoregional anaesthesia. In patients with no pain at follow-up $30 \%$ had received locoregional anaesthesia, either alone or in combination with general anaesthesia. The effect of not having received locoregional anaesthesia did not reach significance in the multivariate analyses, although a trend was apparent $(p=0.089)$. Because of the small numbers and variation of the types of operations included, no real conclusions can be drawn from this finding, but future studies should more rigorously examine the possibility that locoregional anaesthesia may be protective against longterm pain.

In conclusion, the present analyses of the 12 months follow-up data corroborate our previous results. The finding that operations with a long duration (i.e., $>3$ hours) 


\section{CHATTER}

and high levels of acute postoperative pain increase the chance of adverse longterm outcome appears to be robust. This was found across different outcome variables and was consistent for the analyses on the data collected at 6 and 12 months post surgery. We previously speculated that the reason for the higher risk of adverse outcome as a result of longer operations and more intense acute postoperative pain could be due to prolonged and intense noxious peri-operative input and consequently increased central sensitization. The finding that poor preoperative physical condition as reflected by the ASA status may also increase the risk of adverse outcome was substantiated by the present findings. Finally, the role of psychological variables, and most notably surgical fear, was also confirmed although the effects appear to be less robust and more modest than for the other predictors identified. In sum, the consistency of the present findings with those of our first follow-up study attests to the validity and robustness of the predictors previously identified. 


\section{REFERENCES}

1. Macrae WA. Chronic pain after surgery. Br J Anaesth 2001; 87:88--98.

2. Kehlet $H$, Jensen TS, Woolf CJ. Persistent postsurgical pain: risk factors and prevention. Lancet 2006; 367:1618--1625.

3. Peters ML., Sommer M, de Rijke, JM, Kessels A, Heineman E, Patijn J, Marcus MAE, Vlaeyen JWS, van Kleef M. Somatic and psychological predictors of long-term unfavourable outcome after surgical intervention. Ann Surgery 2007; 245: 487--494.

4. Sullivan MJL, Bishop SR, Pivik J. The Pain Catastrophizing Scale: Development and validation. Psychol Assessment $1995 ; 7: 524--532$.

5. Carver CS, White TL. Behavioral inhibition, behavioral activation, and affective responses to impending reward and punishment: The BIS/BAS Scales. J Pers Soc Psychol 1994; 67:319--333.

6. Rand Health Science Program. Rand 36-item Health Survey Manual. In: Rand, ed. Santa Monica, 1992.

7. Kleinbeck SV. Self-reported at-home postoperative recovery. Res Nurs Healt $2000 ; 23: 461--472$. 


\section{Chapter 7}

\section{Acute pain treatment: Do we need a shift in direction?}

Michael Sommer MD, Anne Françoise Gabriel, Jacob Patijn MD PhD,

Maarten van Kleef MD PhD, Hans Fritz Gramke MD, Marco A. E. Marcus MD PhD

Submitted to European Journal of Anesthesiology

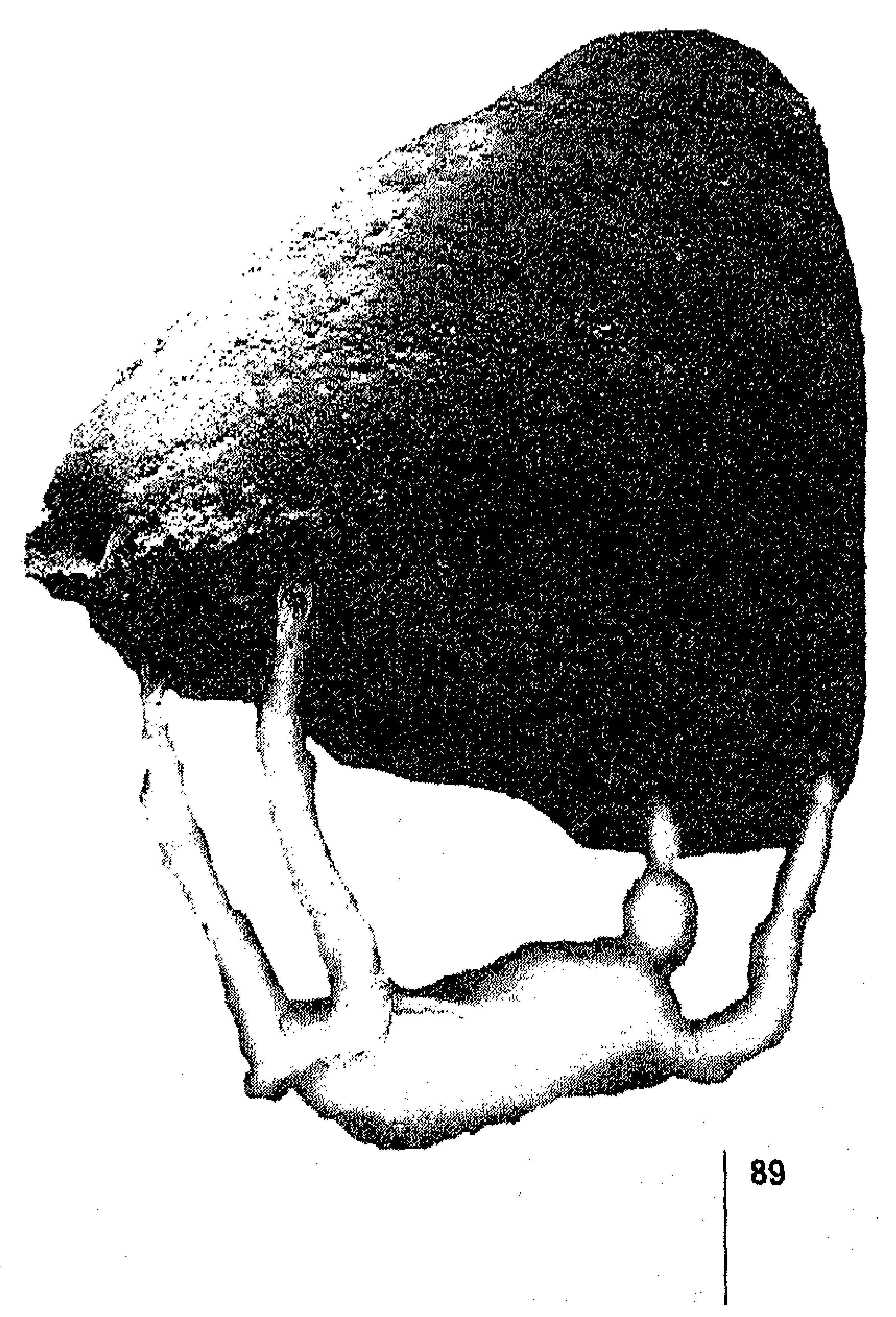




\section{CMAPTER}

\section{INTRODUCTION}

"Effective postoperative pain management remains a significant clinical issue despite major efforts of regulatory agencies and professional societies to establish clinical practice guidelines" (1). This is a common introductory sentence in articles about postoperative pain. A number of studies have demonstrated the extent of the problem: one in three patients experiences pain of more than 3 on a 10 point Visual Analogue Scale (VAS) (2-6). More severe acute pain is not only associated with increased morbidity (7-9) and longer hospital stay, the risk of developing chronic pain after surgical intervention is increased if the postoperative pain is treated insufficiently (10).

\section{Strategies so far: Acute Pain Service}

To improve postoperative pain therapy different attempts were made in terms of logistic adaptation, treatment guidelines or pharmacological innovations. An Acute Pain Service (APS) was established. Dedicated health workers should focus exclusively on that topic of care followed by studies evaluating the effect of an APS. A regular assessment and documentation of pain, dedicated personnel, an algorithm for postoperative pain treatment 24 hours a day 7 days a week are the basic quality criteria of an APS. In 1995 two studies involving the number of hospitals in the USA running an APS were published. Warfield et al. found that $42 \%$ of the interviewed hospitals had an APS whereas Ready et al. found that to be $73 \%(11,12)$. This discrepancy is due to different interpretation of what defines an APS. In a nationwide German survey in $200236 \%$ of all responding hospitals were said to run an APS (13). A more detailed examination of the basic quality criteria revealed that only $50 \%$ of the hospitals met these requirements.

It is even more difficult to estimate the effect on comfort and morbidity because of the pain service. Werner et al. in a meta-analysis of 84,097 patients found lower VAS' in hospitals with APS compared to those without (14). The studies could not be compared with respect to overall outcome in this meta-analysis because of different methodologies. Miaskowski and co-workers found significant differences in postoperative pain, side effects of pain treatment and patient satisfaction in patients who were treated by APS compared to those without APS (15). Pain and side effects were less, with an increased satisfaction score, in the APS treated group.

If a pain service is anaesthesiologist-based, patients are treated with more advanced analgesic techniques such as Patient Controlled Analgesia or epidural catheters. An APS run by anaesthesiologists is expensive. Therefore often only patients requiring more sophisticated techniques are treated. On the other hand, in order to increase the quality of postoperative pain all postoperative patients should be treated by APS regardless of the applied analgesic techniques. An alternative would be a nurse-based APS. Stadler et al. could demonstrate the effect of implementation of a nurse-based APS on treatment quality and costs (16). The advantages of running the APS by nurses is more manpower and lower costs. The authors calculated the hospi- 
tal's costs per day per patient to average 19 Euro with the most expensive day being the first postoperative day.

It is unclear whether an APS has an impact with respect to the length of hospital stay. Lee and co-workers in a meta-analysis included 9 studies involving 14,774 patients to calculate the cost benefit ratio of an APS (17). They concluded that there is a lack of high-quality economic studies to support any cost-effectiveness and costbenefit of an APS.

In conclusion, APS can help to reduce pain in postoperative patients. It should be nurse-based and anaesthesiologist-supervised to reduce costs and increase the number of monitored patients. Its impact on patient outcome is unclear.

\section{Strategies: Treatment guidelines}

Organisational adjustments are just one facet of pain management. Treatment guidelines could contribute to improvement as well. In 2004 an updated report of the ASA Task Force on Acute Pain Management was published to provide the caregiver with guidelines (18). Unfortunately, the recommendations were quite general. The type of surgery, expected postoperative pain level and underlying medical condition should be taken into consideration. This should be incorporated into an analgesic plan. Continuous application of analgesics would be preferable to on demand analgesia. But there are no recommendations with respect to each specific procedure and there is no information as to whether a specific operation was of minor, intermediate, or major surgery. The European Task Force developed more specific recommendations (www.postoppain.org 2007) covering 8 surgical procedures (herniorraphy, laparoscopic cholecystectomy, abdominal hysterectomy, thoracotomy, total knee arthroplasty, total hip arthroplasty, open colonic resection). To arrive at best practice recommendations the recent literature is scanned following a well described methodology. There is an update every 2 years. However, a very limited amount of operations is discussed. Other operations should be treated likewise. In our own study we could demonstrate that similar procedures do not necessarily behave in the same way (predictors, submitted). Factors such as anatomical region and pre-existing pain play an important role in postoperative pain experience. Furthermore, in the preoperative assessment of the PROSPECT recommendations psychological items like catastrophizing, fear for surgery or the expected postoperative pain level were not taken into account.

Thus to improve pain experience after surgical intervention organisational (APS) and structural (guidelines) innovations were made. Until now they have not been able to even approximate the goal of the Audit Commission (UK) of 1997. This commission proposed a standard whereby less than $5 \%$ of patients should experience severe pain by the year 2002 (Audit Commission Anaesthesia under Examination, Audit Commission, London 1997). 


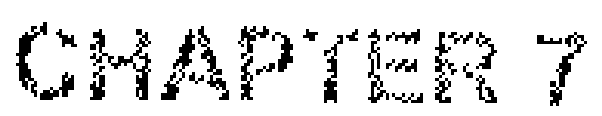

\section{Pharmacological intervention}

An investigation into the pharmacological aspect of the situation is warranted. Can we change pain experience by applying new drugs or different ways of application? Some studies indeed showed a slightly better outcome using new or other drugs in combination or alone (19-22), others couldn't detect any difference (23). The route of application is under investigation as well (24-28). Many studies like our own demonstrated a significant improvement of postoperative pain treatment by continuous regional anaesthesia techniques in comparison with general anaesthesia alone $(24,25,29)$. Nevertheless, in a huge prevalence study in our hospital with 1490 postoperative patients we found $30 \%$ of all patients experienced moderate (VAS above $30)$ or severe pain (VAS above 70) postoperatively (30). This was despite an Acute Pain Service and an acute pain protocol.

\section{Polymorphism and drug-drug-interaction}

Another interesting aspect is alteration of drug effects. Genetic influences in pharmacokinetic pathways seem to play an important role. In the last 10 years a lot of publications have focused on the problem of polymorphism (45-47). About 60 percent of all drug metabolisms take place in the liver. Cytochrome $P_{450}$ system $\left(C_{450}\right.$ ) is responsible for metabolizing around $60 \%$ of these drugs by oxidative processes. $\mathrm{CYP}_{450}$ consists of many iso-enzymes, of which the most important are 2D6, 2C19 and $3 \mathrm{~A} 4$. Codeine was one of the first analgesics recognized as producing adverse drug effects. Codeine as a pro drug is metabolized by the isoenzyme 2D6 into its active form. 2D6-iso-enzyme is responsible for almost $25 \%$ of all drugs metabolized by the CYP 450 system. When the activity of this iso-enzyme is high or very high the concentration of metabolites in the serum increases rapidly. If the activity is low or very low the drug is metabolized slowly or not at all. This can lead to very high or very low blood-drug concentrations. Other important central analgesics that are metabolized via 2D6-iso-enzyme are oxycodone and hydromorphone (48). The 2D6-isoenzyme is not the only pathway that can vary. $\mathrm{M \mu}$-Opioid-receptor-polymorphism also plays a role (49) albeit unclear in what way (50). This is not only important for acute but for chronic pain patients as well.

In addition to polymorphism, drugmdrug interaction is also of interest. Kennedy and co-workers found that $50 \%$ of the 1025 patients admitted for surgery were taking medicines unrelated to surgery (51). On average these patients received 9 different drugs. Taking a drug unrelated to surgery increased the risk of postoperative complications. Drugs can induce or inhibit iso-enzyme of the CYP 450 system, they can function as substrates, or they can be both, an inducer and an inhibitor of the same CYP $_{450}$ iso-enzyme. The beta-blocker pindolol, for example, inhibits 2D6. Administration of codeine to a patient who is on pindolol therapy results in no analgetic effect at all because no analgetic active metabolite can be formed from codeine. On the other hand dexamethasone is an inducer of 2D6. The combination of codeine with dexamethasone leads to a much higher blood concentration of the codeine metabolites 
morphine and norcodeine with possible side effects. Drug-drug interaction can probably be responsible for insufficient pain therapy. It is imaginable that in the future it will be significant to first check drugs for possible interaction before applying them. Nevertheless more research with respect to interaction and polymorphism of different drugs is necessary in the future.

\section{Predictors of patients at risk}

In view of the disappointing results regarding improvement of acute pain therapy possible predictors were investigated. What makes one patient at risk for experiencing more pain than another? Kalkman et al. evaluated in a cohort of 1460 patients the appearance of moderate and severe postoperative pain and found an incidence of $26 \%$ (31). Age, gender, pre-existing pain, incision size, and the kind of procedure itself were correlated with more intense pain experience afterwards. Female sex turned out to be a strong predictor of postoperative pain. Concerning gender, Sullivan and co workers in 2000 demonstrated an important statistical effect: if you correct your data for catastrophizing sex no longer plays a role. In our own study we found no sex differences either after correcting the data for catastrophizing ('Predictoren' submitted). Therefore it is the degree of catastrophizing, registered with the Pain Catastrophizing Scale (PCS) that is an important predictor. The PCS is a validated instrument and its effect on pain nowadays is obvious (32). On the other hand no study has demonstrated an increased use of analgesics if the PCS score was high. Thus a higher PCS is correlated with an increased VAS but not necessarily with an
increased demand for analgesics.

Another possible predictor of postoperative pain is age. The data is conflicting. In one of our studies the middle age group $(40-60)$ had a higher risk of postoperative pain than the younger or older clusters (31). Beauregard et al. did not find any difference with age in day-case surgery (33). Problems with their study were the small sample size and a maximum age of 59 years. This is an important limitation because the age difference is significant in some studies from 60 years on. Macintyre and Jarvis found age to be the best predictor during the first 24 hours postoperatively (34). In their retrospective analysis they found the younger patients suffered from more pain than the older ones. A decrease in volume of distribution and in clearance of morphine, as well as a lower plasma albumin in the elderly, could be the cause of this phenomenon. Likewise Gramke and co-workers in their analysis of pain after day-case surgery found patients less than 45 years of age to be prone to more pain (submitted).

If patients suffer from pain preoperatively the risk of more postoperative pain increases on all postoperative days. Kalkman and co workers demonstrated this for the first eight hours in the Post Anaesthesia Care Unit (31). Thomas et al could detect preoperative pain as a strong predictor for postoperative days (POD) 1 to 5 in patients after hip- or knee-arthroplasty and after neuronal decompression (35). We could verify preoperative pain as predictor for POD 1 to 5 in all patients independent of the kind of operation (30). The same is true for the item expected pain, which 


\section{Chang}

means that the patient expects high pain scores after the operation (article accepted Clin J Pain).

In our study we detected some more predictors. However these items were never statistically significant on all POD's. Thus, careful analysis of the data from predictor studies revealed some hard risk factors: preoperative pain, expected pain and pain catastrophizing. The difficulty lies in how to incorporate this knowledge into clinical practice. These patients obviously need a more tailor-made therapy and a closer monitoring, but is it useful to provide a kind of Intensive Pain Therapy Service? The reason for the insufficient pain therapy is not clear yet. What makes the patients susceptible for more pain? And what is the causality between psychological factors such as increased Pain Catastrophizing Scores or higher Expected Pain and more pain postoperatively? And thirdly, if there are connections between expectations and pain could we influence the expectations positively in one way or another?

\section{Healing environment}

In paediatric medicine it is quite common to create a home-like atmosphere in order to positively influence the hospital experience and pain. It is logical to give the child, in an uncertain situation like a hospital, the security and feeling of well-being that parents provide. To make the child more at ease the surroundings resemble a child's room with toys, computers and movies etc. Given this it is astonishing that it took so long to investigate this effect on adults as well. Being sick or in pain is scary. One could assume that reducing fear could probably improve the healing effect and eventually reduce pain. Interestingly, the use of an 'enriched' or 'healing' environment has shown compelling results in the management and treatment of postoperative pain, in the clinic and also in experimental pain models. When housed in an enriched environment (i.e. physical and social enrichment), rats recover significantly faster from knee inflammatory pain than when housed in a standard individual cage (JM Tall 2005; unpublished results from our lab). Likewise, in the clinic, the importance of the psychosocial environment of pain patients has been demonstrated several times (36). Patients assigned a hospital room with a window view on nature had shorter hospital stays, took fewer analgesics and reported less negative comments in nurses' notes than those assigned in a room with a window facing a brick building wall. According to the idea that the patient's whole environment (from physical to social aspects) is important, several attempts have been undertaken lately in developing multidimensional treatment for pain patients (37-39).

In line with this, a few hospitals in the world have already developed a so-called healing environment for their patients. Such an environment aims at stimulating patients in order to ease and speed up one's recovery. First, from a physical point of view, stimulation of the patient's five senses has been shown to significantly decrease the intensity and/or duration of pain, e.g. using natural lighting and garden views $(40,41)$, via musical therapies $(42)$, through the use of natural components for walls, seats or blankets, etc. 
De Wied and Verbaten evaluated whether vision is capable of influencing the emotion and consequently pain experience. They exposed male subjects to pleasant, neutral, or unpleasant pictures. Simultaneously cold pressure tests were carried out to measure pain tolerance. Pain tolerance was higher when probands were viewing pleasant pictures. In a second experiment they confronted the test persons with pictures that either did or did not include pain-related material and exposed them to cold water at the same time. If the picture included pain cues the cold water was tolerated for a shorter time than if it did not include pain cues (40). This observation is not new. In the early 1990's pain influence experiments had already been carried out. Pain relevant anxiety increased pain, pain irrelevant anxiety decreased pain (43). Similarly reading positive, neutral or negative statements could influence pain tolerance measured by the cold pressure test as well (44). It is likely that it is more than just distraction from pain exposure that influences pain experience. The kind of distraction seems to play a role as well and determines the direction of change. One can argue that lying in a hospital room with other freshly operated patients around you, patients in pain and fear, is not a comforting and reassuring environment.

Based on these findings, the use of a healing environment in Acute Pain Services is strongly advocated, even though the underlying biological mechanism is still unclear.

\section{Summary}

Many efforts have been made thus far to improve acute pain treatment. Organisational aspects were taken into account and APS' were established and monitored. Guidelines were published to help the caregiver. Predictors were found which could identify patients at risk and healing environments were investigated. Pharmacological advances were studied together with genetic disturbances as a reason for therapy failure or unwanted side effects. No single step could turn the tide. The problem is obviously complex which suggests that the answer is complex as well. Generally, five steps must be taken to improve pain release.

1. An APS is useful and it should not be allowed to disappear because of cost reduction. The tasks of the APS should be revised: it is not only the group of patients with access to advanced techniques, which should profit from the service. All postoperative patients should be monitored and the treatment eventually adjusted.

2. The guidelines must be extended.

3. Predictors must be determined in every patient. Most of them are easy to obtain. The anaesthesiologist knows the anatomical region of surgical intervention. Preexisting pain and high pain expectation can be queried. Pain catastrophizing can be estimated using a questionnaire, which is completed by the patient preoperatively. Possible patients at risk should be monitored very closely.

4. The healing environment is quite new. First clinical data are compelling. Some hospitals are already equipped with such an environment. It should be part of the therapy concept to create a cosy and pleasant environment for each patient and 


\section{CHARTER?}

his relatives. Nevertheless more data are necessary and research must be done with respect to the underlying mechanism.

5. Pharmacological research is ongoing. New drugs as well as data about drugdrug-interaction and the meaning of genetic variation are continuously being published. But it is important to incorporate new pharmacological and biological knowledge into medical practice.

For each individual patient the following approach should be applied:

- During preassessment ask the patient to fill in a questionnaire about PCS. Ask him about pre-existing and postoperative expected pain and ask him about the amount of anxiety experienced. If PCS, pre-existing or expected pain is high pay extra attention. Stress the positive effects of regional anaesthesia on postoperative pain therapy. Check his medications for possible interaction with narcotics and analgesics.

- After admission create a safe and pleasant environment for the patient. Introduce the pain nurse who will care for him afterwards.

- If the patient keeps experiencing pain more than 4 on an NRS or VAS despite the positive environment, with his relatives near him, no possible interaction, and all efforts carried out so far, consider changing drugs because of possible polymorphism. 


\section{REFERENCES}

1. Miaskowski C. Patient-controlled modalities for acute postoperative pain management. J Perianesth Nurs 2005;20:255-67.

2. Dolin SJ, Cashman JN, Bland JM. Effectiveness of acute postoperative pain management: I. Evidence from published data. Br J Anaesth 2002;89:409-23.

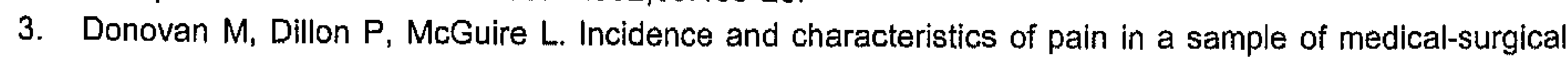
inpatients. Pain 1987;30:69-78.

4. Oates JD, Snowdon SL, Jayson DW. Failure of pain relief after surgery. Attitudes of ward staff and patients to postoperative analgesia. Anaesthesia 1994;49:755-8.

5. Svensson I, Sjostrom B, Haljamae H. Assessment of pain experiences after elective surgery. J Pain Symptom Manage 2000;20:193-201.

6. Cohen FL. Postsurgical pain relief: patients' status and nurses' medication choices. Pain 1980;9:265-74.

7. Ergina PL, Gold SL, Meakins JL. Perioperative care of the elderly patient. World J Surg 1993;17:192-8.

8. Duggleby $W$, Lander J. Cognitive status and postoperative pain: older adults. J Pain Symptom Manage 1994;9:19-27.

9. Joshi GP, Ogunnaike BO. Consequences of inadequate postoperative pain relief and chronic persistent postoperative pain. Anesthesiol Clin North America 2005;23:21-36.

10. Katz J. Pain begets pain: predictors of long term phantom limb pain and postthoracotomy pain. Pain Forum 1997;6:140-4.

11. Warfield $\mathrm{CA}$, Kahn $\mathrm{CH}$. Acute pain management. Programs in U.S. hospitals and experiences and attitudes among U.S. adults. Anesthesiology 1995;83:1090-4.

12. Ready LB. How many acute pain services are there in the United States, and who is managing patientcontrolled analgesia? Anesthesiology 1995;82:322.

13. Stamer UM, Mpasios N, Stuber F, Maier C. A survey of acute pain services in Germany and a discussion of international survey data. Reg Anesth Pain Med 2002;27:125-31

14. Werner MU, Soholm L, Rotboll-Nielsen $P$, Kehlet $H$. Does an acute pain service improve postoperative outcome? Anesth Analg 2002;95:1361-72, table of contents.

15. Miaskowski $C$, Crews J, Ready LB et al. Anesthesia-based pain services improve the quality of postoperative pain management. Pain 1999;80:23-9.

16. Stadler $M$, Schlander $M, B$ raeckman $M$ et al. A cost-utility and cost-effectiveness analysis of an acute pain service. J Clin Anesth 2004;16:159-67.

17. Lee A, Chan S, Chen PP et al. Economic evaluations of acute pain service programs: a systematic review. Clin J Pain 2007;23:726-33.

18. Practice guidelines for acute pain management in the perioperative setting: an updated report by the American Society of Anesthesiologists Task Force on Acute Pain Management. Anesthesiology 2004;100:1573-81.

19. Sun T, Sacan $O$, White PF et al. Perioperative versus postoperative celecoxib on patient outcomes after major plastic surgery procedures. Anesth Analg 2008;106:950-8, table of contents.

20. Ryu JH, Kang MH, Park KS, Do SH. Effects of magnesium sulphate on intraoperative anaesthetic requirements and postoperative analgesia in gynaecology patients receiving total intravenous anaesthesia. Br J Anaesth 2008;100:397-403.

21. Meunier A, Lisander B, Good L. Effects of celecoxib on blood loss, pain, and recovery of function after total knee replacement: a randomized placebo-controlled trial. Acta Orthop 2007;78:661-7.

22. Flood P, Daniel D. Intranasal nicotine for postoperative pain treatment. Anesthesiology 2004;101:141721.

23. Aubrun F, Gaillat $C$, Rosenthal $D$ et al. Effect of a low-dose ketamine regimen on pain, mood, cognitive function and memory after major gynaecological surgery: a randomized, double-blind, placebo-controlled trial. Eur J Anaesthesiol 2008;25:97-105.

24. Richman JM, Wu CL. Epidural analgesia for postoperative pain. Anesthesiol Clin North America 2005;23:125-40.

25. Wu CL, Cohen SR, Richman JM et al. Efficacy of postoperative patient-controlled and continuous infusion epidural analgesia versus intravenous patient-controlled analgesia with opioids: a meta-analysis. Anesthesiology 2005;103:1079-88; quiz 109-10.

26. Power I. Fentanyl $\mathrm{HCl}$ iontophoretic transdermal system (ITS): clinical application of iontophoretic technology in the management of acute postoperative pain. $\mathrm{Br} J$ Anaesth 2007;98:4-11. 


\section{CHABting}

27. Intranasal delivery of morphine may offer better effects. Clin J Oncol Nurs 2003;7:377.

28. Illum L, Watts P, Fisher AN et al. Intranasal delivery of morphine. J Pharmacol Exp Ther 2002;301:391400.

29. Kuo CP, Jao SW, Chen KM et al. Comparison of the effects of thoracic epidurat analgesia and i.v. infusion with lidocaine on cytokine response, postoperative pain and bowel function in patients undergoing colonic surgery. Br J Anaesth 2006;97:640-6.

30. Sommer $M$, de Rijke JM, van Kleef $M$ et al. The prevalence of postoperative pain in a sample of 1490 surgical inpatients. Eur J Anaesthesiol 2008;25:267-74.

31. Kalkman CJ, Visser $\mathrm{K}$, Moen $\mathrm{J}$ et al. Preoperative prediction of severe postoperative pain. Pain 2003;105:415-23.

32. Pavlin DJ, Sullivan MJ, Freund PR, Roesen K. Catastrophizing: a risk factor for postsurgical pain. Clin J Pain 2005;21:83-90.

33. Beauregard $L$, Pomp A, Choiniere $M$. Severity and impact of pain after day-surgery. Can $J$ Anaesth 1998;45:304-11.

34. Macintyre PE, Jarvis DA. Age is the best predictor of postoperative morphine requirements. Pain 1996;64:357-64.

35. Thomas $T$, Robinson $C$, Champion $D$ et al. Prediction and assessment of the severity of postoperative pain and of satisfaction with management. Pain 1998;75:177-85.

36. Ulrich RS. View through a window may influence recovery from surgery. Science 1984;224:420-1.

37. Ettrich U, Seifert J, Scharnagel R, Gunther KP. [A multimodal and multidisciplinary postoperative pain management concept]. Orthopade 2007;36:544, 6-51.

38. Eller U, Dannappel T. [Concept of interdisciplinary pain therapy]. Ther Umsch 1999;56:446-9.

39. Bonifazi $M$, Suman $A L$, Cambiaggi $C$ et al. Changes in salivary cortisol and corticosteroid receptor-alpha mRNA expression following a 3-week multidisciplinary treatment program in patients with fibromyalgia. Psychoneuroendocrinology 2006;31:1076-86.

40. de Wied M, Verbaten MN. Affective pictures processing, attention, and pain tolerance. Pain 2001;90:16372 .

41. Meagher MW, Arnau RC, Rhudy JL. Pain and emotion; effects of affective picture modulation. Psychosom Med 2001;63:79-90.

42. Nilsson U. The anxiety- and pain-reducing effects of music interventions: a systematic review. Aorn J 2008;87:780-807.

43. al Absi M, Rokke PD. Can anxiety help us tolerate pain? Pain 1991:46:43-51.

44. Zelman DC, Howland EW, Nichols SN, Cleeland CS. The effects of induced mood on laboratory pain. Pain 1991;46:105-11.

45. Shanahan EC, Marshall AG, Garrett CP. Adverse reactions to intravenous codeine phosphate in children. A report of three cases. Anaesthesia 1983;38:40-3.

46. Voronov $\mathrm{P}$, Przybylo $\mathrm{HJ}$, Jagannathan N. Apnea in a child after oral codeine: a genetic variant - an ultrarapid metabolizer. Paediatr Anaesth 2007;17:684-7.

47. Gasche $Y$, Daali $Y$, Fathi $M$ et al. Codeine intoxication associated with ultrarapid CYP2D6 metabolism. N Engl J Med 2004;351:2827-31.

48. Thompson $\mathrm{CM}$, Wojno $\mathrm{H}$, Greiner $\mathrm{E}$ et al. Activation of $\mathrm{G}$-proteins by morphine and codeine congeners: insights to the relevance of $\mathrm{O}$ - and $\mathrm{N}$-demethylated metabolites at mu- and deltamopioid receptors. $J$ Pharmacol Exp Ther 2004;308:547-54.

49. Stamer UM, Stuber $F$. The pharmacogenetics of analgesia. Expert Opin Pharmacother 2007;8:2235-45.

50. Janicki $P K$, Schuler $G$, Francis $D$ et al. A genetic association study of the functional $A 118 G$ polymorphism of the human mu-opioid receptor gene in patients with acute and chronic pain. Anesth Analg 2006;103:1011-7.

51. Kennedy JM, van Rij AM, Spears GF et al. Polypharmacy in a general surgical unit and consequences of drug withdrawal. $\mathrm{Br} \mathrm{J}$ Clin Pharmacol 2000;49:353-62. 


\section{Chapter 8}

General discussion and future perspectives

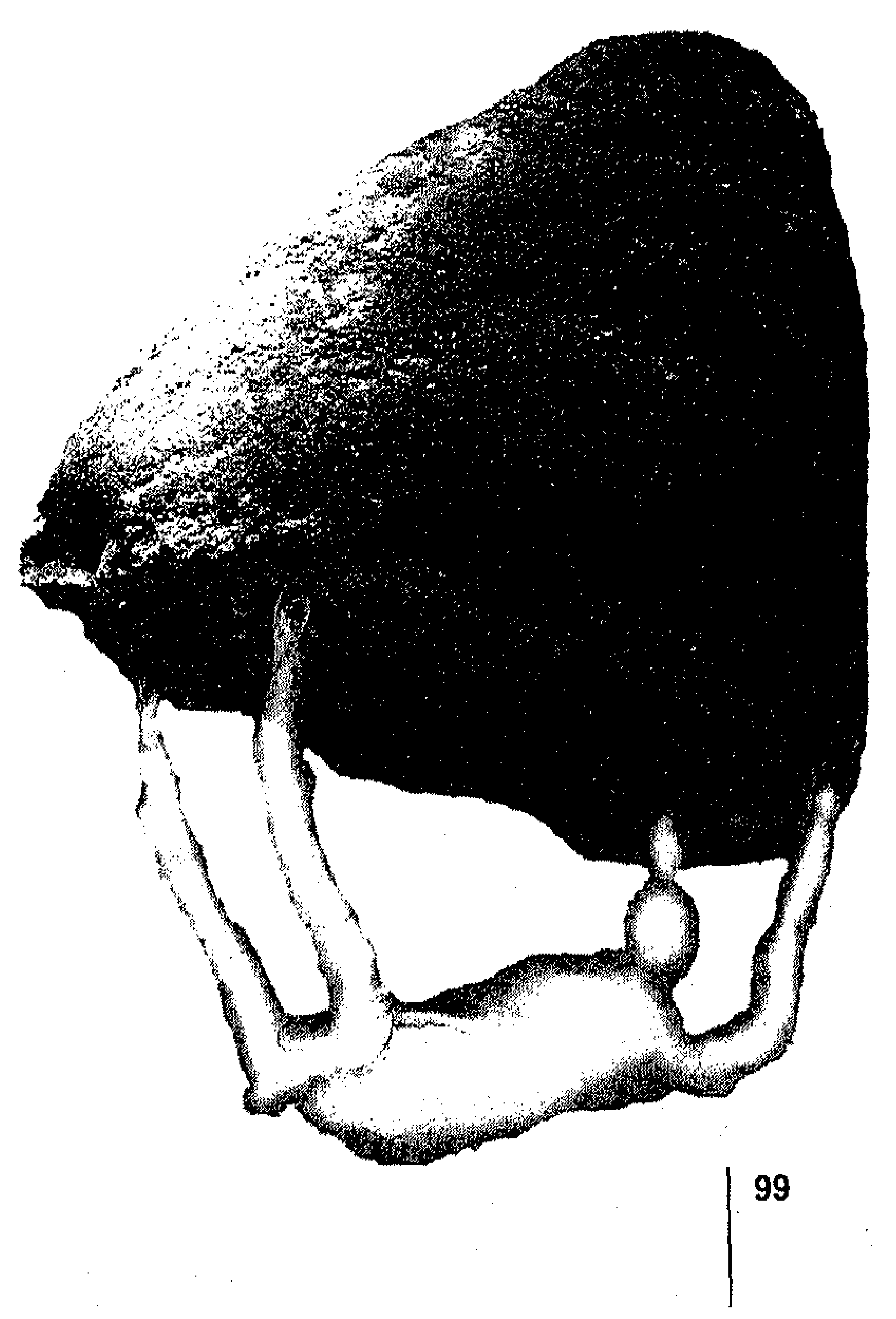




\section{CHAPTEP 8}

If medical knowledge is the product of pathophysiologic research therapeutic conclusions are much easier to draw. For example, from cardiac physiology we know that induction of anaesthesia is stressful for the myocardium because sympathetic stimulation leads to tachycardia, which in turn leads to increased oxygen consumption, which in turn can produce myocardial ischemia in patients with pre-existent coronary artery stenosis. Using a $\beta$-blocker prevents tachycardia and in the end infarction. In this model the underlying mechanism is clear. With this knowledge in mind we are able to formulate practice guidelines which are intended to improve outcome (1). Unfortunately it is not that easy in pain research, the relationship between stimulus and signal is more complex. We know that a painful stimulus is transmitted (via neurons and substances) to the central nervous system and recognized (integrated) as being pain. But why a person experiences more pain than another after exactly the same stimulus in exactly the same place is not known. We now know from clinical research that we can predict the patient population at risk of moderate or severe pain. But what makes the individual patient at risk, and what the causality is, is not known. The risk for developing post surgical persisting pain after 6 months, and chronic pain after 12 months, increases when you have more pain. Does a certain threshold of pain trigger plasticity in the nervous system or are some people more susceptible to a process of plasticity?

\section{QUESTIONS}

What exactly is the relationship between the psychological parameters, we found as predictors, and pain? Is it that patient's who catastrophize experience more pain, because they have a lower pain threshold? Is the fear of pain due to experience of pain in the past because of genetic variation that makes him feel more pain than other subjects? Can pain experience positively be influenced by environmental impulses?

From experimental studies we know that negative expectation can be turned into more acute pain. Benedetti and co-workers, after informed consent, induced pain by applying an inert substance ( $\mathrm{NaCl} 0.9 \%$ ) for 30 minutes duration after having told the patients that it would hurt. Pain increased immediately but after intravenous proglumide, a non-specific cholecystokinin antagonist, pain vanished. Naloxone had no effect on pain (2). This phenomenon is called nocebo effect, meaning, "I shall harm". Nocebo is the opposite of placebo: if you expect a treatment to be painful the outcome will be as expected. Benedetti's study is interesting because it shows the physiologic response of psychological intervention. The question arises whether it is possible to influence pain positively (decrease) without pharmacologically active drugs: the placebo effect. Conscious expectation and unconscious behavioural conditioning leads to release of endogenous neurotransmitters that mimics the expected pharmacological effect. This is the neuro-endocrine background of the placebo effect $(3,4)$. So it is possible to influence the experience of pain verbally (increase) and arouse an endocrine answer. A very important message came from Colloca and co- 
workers who demonstrated that the placebo and nocebo effects can even be enhanced by positive experience (5). The authors induced low tactile, high tactile and low intensity painful stimuli in volunteers before and after verbal influencing and after preconditioning. They showed that telling a person that the stimulus will be very painful produced much more pain than when they were told that it was not that painful. When volunteers were told that a painful stimulus was treated analgetically by another electrode, which was a sham electrode, pain vanished. When they had previously had a positive experience with an analgetic treatment pain vanished even more rapidly.

This study illustrates that effective pain treatment in the patient's history is very important for pain experience and consecutive pain treatment in the future of an individual. Additionally pain experience can be influenced by verbal intervention.

Similarly, the experiments of De Wied and Verbaten, described in chapter VII (6), showed that the visual sense is subject to manipulation as well. Volunteers were exposed to pictures of different contents. If the viewed picture was unpleasant and had something to do with pain the probands in their experiment had lower pain thresholds than if it was a pleasant picture. So obviously it is the hidden information, which is responsible for the neuroendocrine response of no- or placebo. Is this part of the underlying mechanism of pain? And can this knowledge be used for further intervention?

The healing environment actually does nothing other than manipulate the patient in a positive way. The environment is meant to resemble a homely atmosphere to make the patient feel at ease in a rather threatening situation. The aim is to enhance healing and reduce discomfort. From former research we know about the negative consequences of postoperative pain. By reducing postoperative pain morbidity and complications after surgery should be reduced as well. Given the results of the studiesof Colloca, and De Wied and Verbaten, it would be interesting to look at whether it is possible to affect the outcome of patients at risk of more postoperative pain by verbal and environmental interventions. That is: detect patients at risk and prepare them preoperatively by verbal placebo and a cosy environment. Carry out frequent postoperative monitoring for efficacy of pain treatment and apply early and aggressive intervention.

While psychological influence can facilitate the placebo effect, which is due to neurochemical reactions on receptors, conversely, pharmacological intervention can block receptors that are important in pain perception. One pharmacological group with analgesic properties is tricyclic and non-tricyclic antidepressants. Monoaminoxidase plays a role in nociception. Inhibiting monoaminoxidase reuptake in the synaptic cleft by these antidepressants leads to changes in the pain threshold and produces antinociception (7). Pain reactions in the rats were reduced significantly when they received tricyclic or non-tricyclic antidepressants beforehand (SSRI) (8). When the rats were pre-treated with selective a-receptor antagonist (prazosin) or serotonergic receptor antagonist (ondansetron) the analgesic effect of the antidepressants were antagonized. The intention of this study was to localize the target point of antidepressants as analgesics and to identify subtypes of serotonin receptors. Thus, the rats got 


\section{CHAPTER 8}

selective $\alpha$ - and serotonin-antagonists injected into the cerebral ventricles. The authors didn't intend to investigate treatment options in acute pain. Nevertheless the question arises, what is the use of these antidepressants in acute pain treatment if drugs, which are daily practice in anaesthesia, block the antinociceptive effect?

Another very interesting pharmacological group are antiepileptics. As GABA receptor antagonists they block part of the calcium channel (9). Via this channel the neuroexcitatory transmitters, glutamate and substance $P$, which are important in nociception, are excreted and excitation takes place. Inhibiting the calcium channel means reduced excitation and thus reduced nociception. Gabapentin as an antiepileptic is used in chronic pain patients in the long term, yet side effects are common and often limit the use of gabapentin. However, in acute pain patients it was able to reduce the requirement of analgesics without producing a lot of limiting side effects (10).

Without doubt there are a lot of interesting substances but does that mean that we should apply antiepileptics together with antidepressants, $\alpha$-agonists and/or probably some NMDA receptor antagonists? Maybe, however, we need more data, and good studies in order to learn about these drugs and the receptors they inhibit and their mechanism of pain inhibition.

In three decennia we couldn't turn the tide. After an initial reduction in the number of patients with postoperative severe pain, after installing an APS and treatment guidelines, in the last 10 years we stalled. In the United States of America a task force is dealing with this problem. In Europe, The PROSPECT group tries to give a 2 yearly updated guideline for a couple of procedures.

Currently it is rather confusing. Data about a lot of different receptor antagonists have been published together with data about pain catastrophizing, fear for surgery etc.

It may be time to form another task force. All research done so far, together with proposed studies, should be collected. Data about basic physiologic research should be integrated with psychological data and pharmacological studies. In an annual congress, organized by this task force, all results should be presented to get an overview.

What are the most promising results? How to move forward, in the near future, with respect to research and treatment? Which part of the multimodal problem pain needs more investigation?

This could lead to a plan to decrease postoperative pain, and therefore chronic pain, and finally reach the goal originally set in the early nineties (19) to decrease postoperative pain in $90 \%$ of patients under a VAS score of 30 . Another goal we would like to include is to lower the incidence of chronic pain after all operations to less than $5 \%$. This will be a long road, but for the people involved in the journey, it will be rewarding. 


\section{REFERENCES}

1. Fleisher LA, Beckman JA, Brown KA et al. ACC/AHA 2006 guideline update on perioperative cardiovascular evaluation for noncardiac surgery: focused update on perioperative beta-blocker therapy--a report of the American College of Cardiology/American Heart Association Task Force on Practice Guidelines (Writing Committee to Update the 2002 Guidelines on Perioperative Cardiovascular Evaluation for Noncardiac Surgery). Anesth Analg 2007;104:15-26.

2. Benedetti $F$, Amanzio $M$. The neurobiology of placebo analgesia: from endogenous opioids to chole. cystokinin. Prog Neurobiol 1997;52:109-25.

3. Pacheco-Lopez G, Engler H, Niemi MB, Schedlowski M. Expectations and associations that heal: Immunomodulatory placebo effects and its neurobiology. Brain Behav Immun 2006;20:430-46.

4. Benedetti F, Amanzio M, Vighetti $S$, Asteggiano $G$. The biochemical and neuroendocrine bases of the hyperalgesic nocebo effect. J Neurosci 2006;26:12014-22.

5. Colloca $L$, Sigaudo $M$, Benedetti $F$. The role of learning in nocebo and placebo effects. Pain 2008;136:211-8.

6. de Wied M, Verbaten MN. Affective pictures processing, attention, and pain tolerance. Pain 2001;90:16372.

7. Richelson E, Pfenning M. Blockade by antidepressants and related compounds of biogenic amine uptake into rat brain synaptosomes: most antidepressants selectively block norepinephrine uptake. Eur J Pharmacol 1984;104:277-86.

8. Yokogawa $F$, Kiuchi $Y$, Ishikawa $Y$ et al. An investigation of monoamine receptors involved in antinociceptive effects of antidepressants. Anesth Analg 2002;95:163-8, table of contents.

9. Pogatzki-Zahn EM, Zahn PK. [New substances and applications for postoperative pain therapy.]. Schmerz 2008;22:353-69.

10. White PF. The changing role of non-opioid analgesic techniques in the management of postoperative pain. Anesth Analg 2005;101:S5-22. 


\title{
Chapter 9
}

\author{
Summary
}

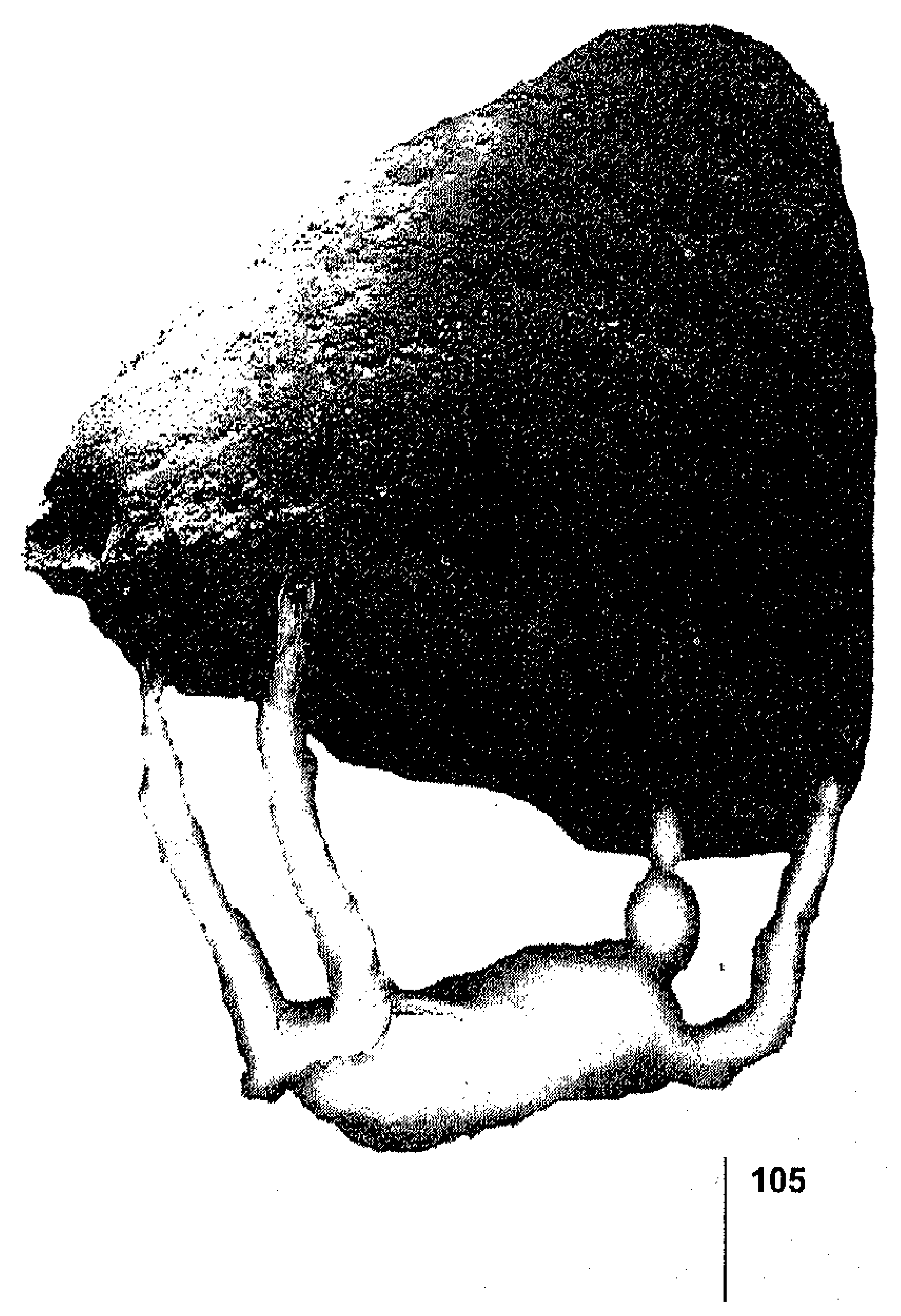




\section{CHAPTES}

The level of postoperative pain in adults during the last decade seems to be unimproved. Despite establishing Acute Pain Services and treatment guidelines the VAS (visual analogue score) remains on an unaltered high level. For the patient, this is of major importance. Continuous pain stimuli trigger the sympathetic system, which in turn causes increased oxygen consumption and can induce organ dysfunction. Another consequence of increased acute pain could be a contribution to higher incidence of chronic pain.

To determine the percentage of patients with moderate or severe pain after surgery in a university hospital, despite an Acute Pain Service and treatment guidelines, we conducted a prospective study that is described in Chapter 2 . The study registered the prevalence of postoperative pain in a huge adult patient population from different surgical departments from the day of operation until day four postoperatively. We conclude that the extent of moderate or severe pain the first 24 hours after surgery is approximately $36 \%$. One hour after the operation this is as high as $41 \%$. The next day postoperatively it is still $30 \%$. From postoperative day 2 to 4 the percentage of patients with moderate or severe pain declines from $19 \%$ on day 2 to 14 $\%$ on POD 4. Particularly, procedures on an extremity or on the spine are more painful than operations in other anatomical regions.

Once it is possible to identify patients at risk one could intensify the attention to these patients postoperatively. Therefore we analyzed our data with respect to predictors of postoperative pain in Chapter 3 . In a multiple regression analysis we looked for somatic factors (pre-existing pain, anatomical site of operation, age and gender), surgical and anaesthesiological factors (operation time and anaesthesiological procedures), and psychological parameters (expected pain, long term fear and pain catastrophizing). We found four strong predictors for moderate or severe postoperative pain, i.e. preoperative pain and expected pain by the patient above 40 , which were statistically significant on all postoperative days, and long term fear and Pain Catastrophizing on day 1 to 3 and day 3 to 4 , respectively.

Obviously it is important to know whether the results of a broad population with different needs and goals can be applied to a smaller homogenous population as well. We analyzed the data of patients that were operated by the Ear-Nose-and throat (ENT) surgeon and searched for prevalence and predictors in this group. In Chapter 4 the data is presented. After multivariate analysis we identified three strong predictors of postoperative pain, which are preoperative pain, pain catastrophizing and anatomical site.

There are suggestions that more acute pain after surgery is associated with an increased risk of the onset of chronic pain. In the literature, incidence of chronic pain after an operation varies but a substantial part of these patients report interference of pain and daily activities leading to physical impairment. So it would be important to identify patients at risk for developing chronic pain after surgery. We contacted the patients from our prevalence study 6 months later and scored the prevalence of chronic pain in order to crystallize somatic and psychological predictors. In Chapter 5 we discuss the results of this survey. Intense acute postoperative pain is indeed a 
predictor of pain after 6 months but there are additional risk factors as well. Long duration of operation, ASA status, and preoperative fear of surgery predict persistent pain 6 months after surgery.

Pain 6 months after surgery doesn't automatically mean that these patients will still be in pain one year after the intervention. In Chapter 6 we describe the results of the 12 months survey in these patients. Interestingly, there are some predictors that are the same for a 6 month and a 12 month period. These are long duration of procedure and high VAS level in the first days after surgery. However, fear for operation and a poor preoperative physical condition expressed as ASA status was also identified although it was less robust and more modest than other predictors.

In Chapter 7 we discuss all the results of recent research and consider a concept consisting of good medical knowledge and some new aspects, which could probably help to improve acute pain therapy.

Finally in Chapter 8 we discuss aspects not previously mentioned and formulate a concept for further research. 
Samenvatting 


\section{CHAPTER 9}

Het aantal volwassen patiënten met verhoogde postoperatieve pijnscores is de laatste decennia niet veranderd. Ondanks het installeren van een Acute Pijn Service en behandelingsrichtlijnen blijft de gemiddelde VAS (Visueel Analoge Score) op een gelijkblijvend hoog niveau. Voor de patiënt is dit van uitermate belang. Continue pijn triggert het sympathische zenuwstelsel, wat op zijn beurt tot een verhoogde zuurstofconsumptie lijdt en zodoende orgaan disfunctie kan veroorzaken. Verder kan toegenomen postoperatieve pijn tot een hogere incidentie aan chronische pijn leiden.

Om het aantal patiënten met moderate of ernstige acute postoperatieve pijn in beeld te brengen hebben we een prospectief onderzoek doorgevoerd dat in hoofdstuk 2 beschreven wordt. Het onderzoek registreert de prevalentie van postoperatieve pijn in een grote volwassen patiëntenpopulatie van verschillende chirurgische afdelingen vanaf de dag van de operatie tot vier dagen erna. We vinden in de eerste 24 uur na de operatie een percentage van gemiddeld $36 \%$ patiënten met moderate of ernstige pijn. Het eerste uur na de operatie bedraagt dit zelfs $42 \%$. De volgende dag, dag 1 na de operatie, is dit nog steeds $30 \%$. Vanaf postoperatieve dag 2 tot en met 4 daalt dit percentage van $19 \%$ op dag 2 tot $14 \%$ op dag 4 . Vooral operaties aan extremiteiten of de wervelkolom veroorzaken meer pijn dan andere ingrepen.

Als het mogelijk zou zijn om risico patiënten te identificeren zou de postoperatieve aandacht voor deze mensen geïntensiveerd kunnen worden. Daarom hebben we onze data in hoofdstuk 3 geanalyseerd met het zicht op mogelijke predictoren van moderate of ernstige postoperatieve pijn. In een multiple regressieanalyse hebben we naar factoren van somatische (preëxistente pijn, anatomische regio van de operatie, leeftijd en geslacht), chirurgische en anesthesiologische (duur van operatie, anesthesiologische technieken), en psychologische (verwachte pijn, lange termijn angst en pijn catastroferen) aard gekeken. We vonden vier harde predictoren van moderate of ernstige postoperatieve pijn, te weten preoperatieve pijn en door de patiënt verwachte pijnscores van meer dan 40 (op een $100 \mathrm{~mm}$ VAS), welke statistisch significant op alle vier postoperatieve dagen waren, en lange termijn angst en pijn catastroferen welke op dag 1 tot en met 3 respectievelijk 3 tot en met 4 statistisch significant waren.

Het is interessant te kijken, of wat voor een grote algemene groep geldt, ook van toepassing is op een kleine geselecteerde patiëntenpopulatie. We hebben daarom onze data opnieuw geanalyseerd met zicht op prevalentie en predictoren en hebben daarvoor de groep van patiënten die een keel-neus-oor-operatie ondergingen uitgezocht. In hoofdstuk 4 presenteren we de data. Na een multivariate analyse konden we drie harde predictoren van meer postoperatieve pijn identificeren, namelijk preoperatieve pijn, pijn catastroferen en anatomische regio.

Er zijn aanwijzingen dat toegenomen acute pijn geassocieerd is met een verhoogde kans op chronische pijn. De literatuur geeft verschillende incidentie van chronische pijn na operaties aan maar bij een aanzienlijk deel van de patiënten met postchirurgisch persisterende pijn of chronische pijn interfereert deze pijn met de alledaagse activiteiten en leidt tot disfunctioneren. Daarom zou het van belang zijn om risico patiënten voor het ontwikkelen van chronische pijn te identificeren. We contacteer- 
den onze patiënten 6 maanden na de operatie opnieuw en registreerden prevalentie van postchirurgisch persisterende pijn om uiteindelijk somatische en psychologische predictoren te identificeren. In hoofdstuk 5 worden de resultaten van dit onderzoek voorgesteld. Intensieve pijn 6 maanden na de operatie is inderdaad een predictor van pijn 6 maanden na de ingreep. Maar er zijn nog andere predictoren. Langere maanden na de ingreep.
matieduur, ASA status en preoperatieve angst voor chirurgie voorspellen pijn 6

Persisterende pijn 6 maanden na de ingreep betekent niet automatisch dat er een jaar na de chirurgische interventie nog steeds pijn bestaat. In hoofdstuk 6 beschrijven we daarom de resultaten van een onderzoek 12 maanden na de ingreep. Het is interessant dat inderdaad sommige predictoren van pijn 6 maanden en 12 maanden na chirurgie hetzelfde zijn. Het gaat hier om lange operatie duur en hoge score op de VAS de eerste dagen na de operatie. Maar angst voor operatie en slechte lichamelijke conditie, uitgedrukt als ASA status, waren eveneens predictoren, al was het in mindere mate dan de anderen.

In Hoofdstuk 7 worden alle resultaten van recent onderzoek besproken en denken we over een concept na dat de medische praktijk en nieuwe aspecten die mogelijk de pijntherapie zouden kunnen verbeteren in overeenstemming brengt.

Tenslotte bespreken we in hoofdstuk 8 aspecten die nog niet aan bod zijn gekomen en formuleren we onderzoeksdoelen voor de toekomst. 


\section{Dankwoord}

Het schrijven van een boek gaat gepaard met een hoge inspanning en nog meer tranen en vloeken (je kan me niet anders vertellen, ik heb het meegemaakt!) van allen, die direct of zijdelings erbij betrokken zijn geraakt. ledereen leest dan ook eerst het dankwoord en zoekt of hij of zij desbetreffend aan bod zijn gekomen. En terecht! Toch is het niet mogelijk om achteraf nog te zeggen wie nou wat en wie het meest heeft bijgedragen. Maar er is een kerngroep, de mensen die bijna dagelijks erbij betrokken waren, meegewerkt hebben aan het verwerken van de data en het tot stand komen van de artikelen, schrijvend en denkend:

Marco Marcus, Jose Geurts, Marjan de Rijke en Madelon Peters, hebben het leeuwendeel en zeker de vermoeiendste delen voor hun rekening genomen. Jaap Patijn, Fons Kessels, Hans-Fritz Gramke, Björn Stessels, Resie Vanderbroeck en Anne Francoise Gabriel hebben een grote bijdrage geleverd bij het schrijven en denken.

Een bijzondere rol speelde Maarten van Kleef, zowel als promotor als collega. De gesprekken waren inspirerend en motiverend en hebben me op gezette tijden weer op de rails gezet. Dat was zeker nodig, temeer omdat je al gaande toch snel de weg kwijt raakt. Daarvoor extra dank!

Een verschrikkelijke taak is en blijft het organiseren van een afdeling om zodoende tijd en ruimte te creëren voor het schrijven van een proefschrift. Dit heeft Ruud Timmer gedaan. I $\mathrm{k}$ denk dat niemand dit beter had gekund, het was een klus!

Uiteindelijk moeten heel veel mensen het ontgelden(?) als je je terugtrekt om te schrijven. De gehele staf van de anesthesie moest steeds weer mijn klinische taken overnemen.

Bijzonder leuk en waardevol was de ondersteuning door de mensen van het secretariaat. Wendy van Engelshoven en Hermina Gielen hebben bovendien ook heel praktisch meegeholpen met het project. Ook daarvoor dank.

De "kindergroep" van de anesthesie moest het het meest ontgelden. Aangenomen ben ik voor de kindergroep en het organiseren van de taken binnen deze groep. Maar er is niet meer veel van gekomen na het begin van dit onderzoek. Nu gaat het weer anders worden en we hebben weer het hoofd vrij voor de kinderzaken, die belangrijk zijn in deze wereld.

Henriette, jij bent klaar met je proefschrift, ik nu ook. Daar gaan we! 


\section{DAWKWOORD}

Nog iemand is van betekenis in dit verhaal ook al is het maar zijdelings. Er zijn vooral twee mensen waar ik van de gesprekken heb genoten en dat nog steeds doe. Jaap Patijn heeft behalve als actief lid van onze research groep, een strenge en intelligente instantie (zie boven), ook nog een belangrijke bijdrage geleverd aan de algemene beschouwingen in het leven en de humor ervan. En Jan Schieveld zet mijn gedachten zo nu en dan op nul door zijn frisse en heel andere kijk op de wereld.

Het was een klus, het is klaar. Nu de anderen. 


\section{Curriculum Vitae}

Michael Sommer was born in Darmstadt, Germany, in 1960. He started Medical School at the Free University of Amsterdam (VU), Netherlands, where he graduated cum laude in 1990. He was trained in anaesthesiology in Düsseldorf and Neuss, Germany, and in 1994 he went to the department of anaesthesiology, intensive care medicine and emergency medicine of the Ernst-Moritz-Arndt-university, Greifswald, Germany, as a staff member for anaesthesiology and intensive care medicine. He worked for 2 years in neuroanaesthesia and started a fellow ship in paediatric anaesthesia thereafter. In 1997 he obtained his conferral of a doctorate (Dr. med.) with the thesis "Evaluation of low dose prostaglandin E1 treatment for ductus dependent congenital heart disease" (promoter Prof. Dr. H.H. Kramer, department of paediatric cardiology, Heinrich-Heine-University, Düsseldorf, Germany) and he became senior staff member of the staff of anaesthesiologists. In 2001 he became assistant head of the department of anaesthesiology and intensive care medicine (Geschäftsführender Oberarzt).

In 2002 Michael Sommer went to Maastricht to become staff member of the department of anaesthesiology and pain therapy with special interest in paediatric anaesthesia. In 2007 he started a training in interventional chronic pain therapy. 


\section{List of publications}

Kramer HH, Sommer M, Ramos S, Krogmann O. Evaluation of low dose prostaglandin E1 treatment for ductus dependent congenital heart disease. Eur J Paediatr $1997 ; 54: 700-707$

Sommer M, Beckert M, Gaab MR, Hachenberg T. Wirkungen von Isofluran oder Mannitol auf die regionale Oxyhaemoglobinbildung (RSO2) des zerebralen Kortx. Anaesthesist 1995; 45:A88

Beckert M, Sommer M, Gaab MR, Piek J. Wirkungen von Mannitol und Hyperventilation auf die regionale Oxyhaemoglobinbildung des zerebralen Kortx. Zentralblatt Neurochir 1995

Riedel T, Rosolski T, Sommer M, Voss J. Intubationsbedingungen und neuromuskuläres Neuromonitoring : Vergleich von Cis-Atracurium und Atracurium. AINS 32 (1997), S90

Mauermann K, Rosolski T, Fastnacht J, Gutezeit A, Sommer M. Qualität von Filtersystemen zur Retransfusion von ungewaschenen autologen Blut. AINS 32 (1997), S90

Sommer M, Runge U, Walter M, Sommer C, Hachenberg T, Horn J. Aktivitätesänderung in der Elektrokortikographie (ECOG) bei Patienten mit pharmakoresistenten fokalen Epilepsien nach intravenöser Propofolinjektion. AINS S3 (1998) S212

Sommer M, Runge U, Jülich $M$, Piek, J, Hachenberg T. Electrocorticographic changes (ECOG) in patients with pharmacoresistent epilpesy after intravenous propofol, Neurosurg Anesth 2000, \$5 


\section{LIST OF PUBLICATIONS}

Zinke $\mathrm{H}$, Sommer M. Thorakale Epiduralanalgesie bei der Schmerzbehandung der schwer verlaufenden Pankreatitis. Intensivmedizin 2000

Sommer M, Riedel J, Fusch Ch, Hachenberg T. Remifentanil in a preterm newborn. Paed Anaesth 2001; 11(2):252-4

Sommer M, Schuldt M, Runge U, Gielen-Wijffels S, Marcus MA. Bilateral hypoglossal nerve injury following the use of the lanryngeal mask without the use of nitrous oxide. Acta Anaesthesiol Scand. 2004; 48(3):377-8

Sommer M., Willigers $H$, Marcus MA. Total intravenous anaesthesia in children. Anaesthesist 2004;53(7):653-5

Sommer M, de Rijke JM, van Kleef M, Kessels AG, Peters ML, Geurts JW, Marcus MA. The prevalence of postoperative pain in a sample of 1490 inpatients. Eur J Anaesthesiol 2008; 25(4):267-74

Gramke HF, de Rijke JM, van Kleef M, Raps, F, Kessels AG, Peters ML, Sommer M, Marcus MA. The prevalence of postoperative pain in a cross-sectional group of patients after day-case surgery in a university hospital. Clin J Pain 2007; 23(6)543-8

Peters ML, Sommer M, de Rijke JM, Kessels F, Heineman E, Patijn J, Marcus MA, Vlayen JW, van Kleef M. Somatic and psychologic predictors of long-term unfavourable outcome after surgical intervention. Ann Surg 2007; 245(3):487-94

Sommer M, Geurts J, Stessel B, Kessels AG, Peters ML, Patijn J, van Kleef M, Kremer B, Marcus MA. Prevalence and predictors of postoperative pain after ear, nose and throat (ENT) surgery. Arch Oto Laryngo 2008 accepted for publication

Gramke HF, Marcus MA, Sommer M, van Kleef M. Postoperatieve pijnbestrijding: richtlijnen, organisatie en technieken. Submitted NTVG

Gramke HF, de Rijke JM, van Kleef M, Kessels AGH, Peters ML, Sommer M, Marcus MAE. Predictive factors of postoperative pain after day case surgery. Submitted Clin J Pain

Sommer M, Gabriel AF, Patijn J, van Kleef M, Gramke HF, Marcus MAE. Do we need a shift in direction. Submitted Eur $J$ Anesthesiol

Sommer M, de Rijke JM, van Kleef M, Kessels AG, Peters ML, Geurts JWJM, Patijn J, Gramke HF, Marcus MAE. Predictors of acute postoperative pain after elective surgery. Accepted Clin J Pain. 